\title{
Synthesis of the C7-C15 trans decalin portion of natural antibiotic Tetrodecamycin
}

\author{
Jeffrey M. Warrington and Louis Barriault* \\ Department of Chemistry,University of Ottawa, 10 Marie Curie, Ottawa, OntarioK1N 6N5, \\ Canada
}

Email: 1barriau@science.uottawa.ca

\section{Table of Contents}

General Experimental S1

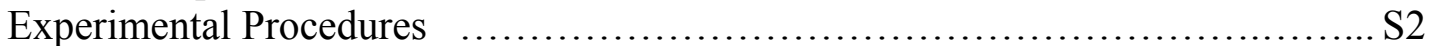

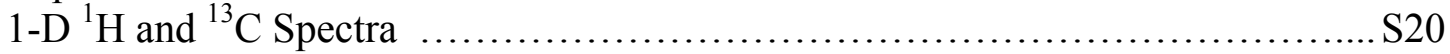

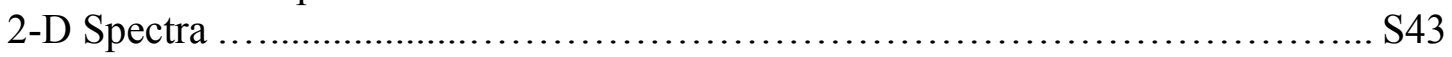

\section{General Experimental}

All reactions were performed under argon in flame-dried glassware equipped with a magnetic stirbar and a rubber septum unless otherwise indicated. Solvents used were freshly distilled prior to use: ether, THF and 1,2-dimethoxyethane (DME) over sodium and benzophenone; dichloromethane, toluene and DMF over calcium hydride. All other commercial reagents were used without purification.

Microwave reactions were preformed using a CEM Model ESP-1500 Plus microwave oven equipped with a pressure monitoring device and an EST-300 Plus fiber optic temperature probe. 
The reaction vessel was a quartz tube, and in each case was added a carboflon ${ }^{\mathrm{TM}}$ to aid in the absorption of microwave radiation.

Reactions were monitored by TLC analysis using glass plates precoated ( $250 \mu \mathrm{m}$ thickness) with silica gel $60 \mathrm{~F}_{254}$ (E. Merck). TLC plates were viewed using UV light, $p$-anisaldehye staining solution, phosphomolybdic acid staining solution or potassium permanganate staining solution. Flash chromatography was carried out on 230-400 mesh silica gel 60.

${ }^{1} \mathrm{H}$ and ${ }^{13} \mathrm{C}$ NMR spectra were recorded on Bruker Avance $300 \mathrm{MHz}$, Bruker Avance $500 \mathrm{MHz}$ or Varian INOVA $500 \mathrm{MHz}$ spectrometers in the specified deuterated solvent. IR spectra were recorded on a Bomen Michaelson 100 FTIR spectrometer. HRMS spectra were obtained using a Kratos Analytical Concept spectrometer, and melting points were recorded using a Gallenkamp P1106 g Melting Point Apparatus.

\section{Experimental Procedures}

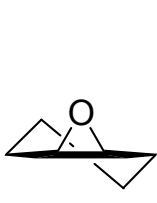

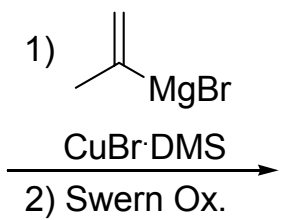

$76 \%$ overall

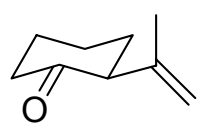

6

2-Isopropenyl-cyclohexanone (6). A clean, dry 1L roundbottom flask fitted with a large magnetic stirring bar was flame-dried under vacuum and allowed to cool. The flask was backfilled with Argon, charged with $\mathrm{Cu}(\mathrm{I}) \mathrm{Br}$ dimethyl sulfide (2.6 g, $0.013 \mathrm{~mol}$ ), and dry THF $(200 \mathrm{~mL})$. After fitting with a septum and balloon, the slurry was stirred for a few minutes prior to immersion in an isopropanol dry ice bath at $-50{ }^{\circ} \mathrm{C}$. Once cool, isopropenyl magnesium 
bromide (500 mL, $0.250 \mathrm{~mol}$ ) was added over 20-30 minutes to give the reaction mixture an orange colour. The reaction was allowed to warm to $-30{ }^{\circ} \mathrm{C}$ and cyclohexene oxide $(30.4 \mathrm{~mL}$, $0.250 \mathrm{~mol}$ ) was added dropwise. The mixture was allowed to warm to room temperature over 3 hours at which point it had taken on a dark rum colour. The reaction was quenched with $\mathrm{NH}_{4} \mathrm{Cl}_{\text {(sat) }}(100 \mathrm{~mL})$ at $0{ }^{\circ} \mathrm{C}$. Air was bubbled through the mixture for 2 hours and it was filtered through paper. Extraction of the organic phase ( $\left.4 \times 50 \mathrm{~mL} \mathrm{Et}_{2} \mathrm{O}\right)$, drying over $\mathrm{MgSO}_{4}$ and removal of the volatiles gave a yellow oil which was used for the next without further purification.

A clean dry flask under argon fitted with a magnetic stirrer was charged with bulk grade dichloromethane $(500 \mathrm{~mL})$ followed by oxalyl chloride $(38.3 \mathrm{~mL}, 0.375 \mathrm{~mol})$. The mixture was cooled to $-78{ }^{\circ} \mathrm{C}$ and DMSO (62 mL, $0.75 \mathrm{~mol}$ ) was added very slowly. (CAUTION: copious amounts of gas are produced and extra venting must be provided). After 30 minutes, a solution of the crude alcohol ( $0.250 \mathrm{~mol}$ maximum) in dichloromethane $(100 \mathrm{~mL})$ was added and allowed to stir. After $2 \mathrm{~h}$, a white-yellow precipitate had formed, and $\mathrm{Et}_{3} \mathrm{~N}(0.75 \mathrm{~mol})$ was added to give a thick yellow sludge sludge. The slurry was allowed to warm to room temperature, and agitated manually periodically over $1 \mathrm{~h}$. Water $(100 \mathrm{~mL})$ was added, and the aqueous layer was extracted with dichloromethane ( $3 \times 100 \mathrm{~mL})$. The combined organic (bottom) layers were dried over $\mathrm{MgSO}_{4}$ and concentrated. The crude was redissolved in diethyl ether $(150 \mathrm{~mL})$ and filtered to remove the accumulated ammonium salts. After removal of the volatiles, the orange residue was dry packed on silica gel ( $\sim 30 \mathrm{~g}$ ) and subjected to flash chromatography. (0 to 15\% EtOAc in 3 steps) to give 6 as a pale yellow oil. (32.0 g, 76\%). Spectral data available from Barriault, L.; Warrington, J. Yap, G. P. Org. Lett. 2000, 2, 663

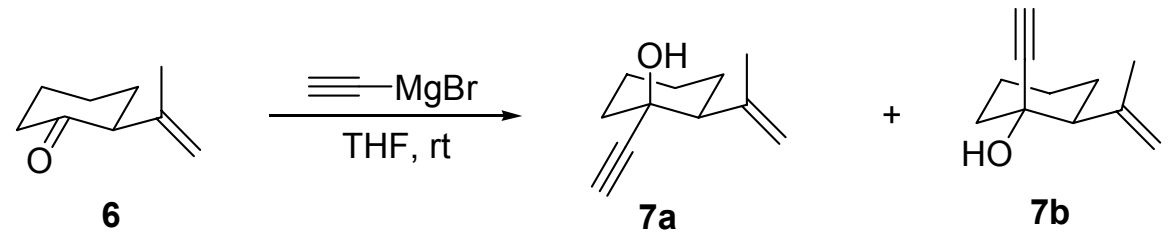

1-Ethynyl-2-isopropenyl-cyclohexanol (7). A clean, dry 1L flask fitted with magnetic stirrer was flame dried and placed under argon. The flask was charged with ether $(200 \mathrm{~mL})$ and ketone $6(32.0 \mathrm{~g}, 0.232 \mathrm{~mol})$ and cooled to $0{ }^{\circ} \mathrm{C}$. Ethynyl magnesium bromide $(0.5 \mathrm{M}$ solution in 
THF, $600 \mathrm{~mL}$ ) was added and the reaction was allowed to warm to room temperature over 4 hours. The mixture was recooled to $0{ }^{\circ} \mathrm{C}$ and quenched with saturated $\mathrm{NH}_{4} \mathrm{Cl}_{(\mathrm{aq})}(100 \mathrm{~mL})$. The organic layers were extracted with ether $(4 \times 75 \mathrm{~mL})$ dried over $\mathrm{MgSO}_{4}$ and concentrated. The mixture was passed through a short path of silica (20\% Ether in Hexanes) to give $\mathbf{7 a , b}$ as a yellow oil (34.9 g) as a 1.5:1 mixture of diastereomers (GC-MS). $\left(\mathrm{rF}\right.$ in $30 \% \mathrm{Et}_{2} \mathrm{O}$ in Hexanes: trans ring junction: 0.6, cis ring junction: 0.55). A small amount of the desired trans ring junction was repurified for characterization to give $7 \mathbf{a}$ as a colorless oil.

${ }^{1} \mathrm{H} \mathbf{N M R}\left(\mathrm{CDCl}_{3}, 300 \mathrm{MHz}\right) \delta_{\mathrm{ppm}}=5.20(\mathrm{br} \mathrm{s}, 1 \mathrm{H}), 5.03(\mathrm{br} \mathrm{s}, 1 \mathrm{H}), 2.39(\mathrm{~s}, 1 \mathrm{H}), 2.25-2.09(\mathrm{~m}$, $3 \mathrm{H}), 1.95(\mathrm{~s}, 3 \mathrm{H}), 1.75-1.42(\mathrm{~m}, 6 \mathrm{H}), 1.35-1.07(\mathrm{~m}, 1 \mathrm{H}) .{ }^{13} \mathbf{C} \mathbf{N M R}\left(\mathrm{CDCl}_{3}, 75 \mathrm{MHz}\right) \delta_{\mathrm{ppm}}=$ 148.5 (C QUAT.), 112.7 ( $\mathrm{CH}_{2}$ ), 89.0 (C QUAT.), 71.6 (CH), 67.3 (C QUAT.), 52.8 (CH), 40.0 $\left(\mathrm{CH}_{2}\right), 27.0\left(\mathrm{CH}_{2}\right), 26.2\left(\mathrm{CH}_{3}\right), 26.0\left(\mathrm{CH}_{2}\right), 20.8\left(\mathrm{CH}_{2}\right)$. IR (FTIR, neat) $v=3734(\mathrm{~s}), 2924(\mathrm{~s})$, $1558,1540,1507 \mathrm{~cm}^{-1}$. HRMS (EI) Expected for $\mathrm{C}_{11} \mathrm{H}_{16} \mathrm{O}_{1}\left[(\mathrm{M})^{+}\right]$: 164.12012, found: 164.11865 $(2.11 \%)$
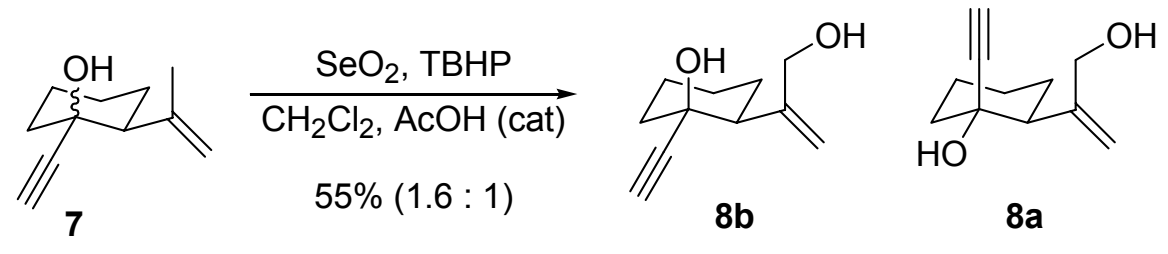

\section{1-Ethynyl-2-(1-hydroxymethyl-vinyl)-cyclohexanols (8a and 8b). A dry 1L}

roundbottom flask fitted with a magnetic stirrer was charged with dichloromethane $(300 \mathrm{~mL})$ under argon. $\mathrm{SeO}_{2}(11.7 \mathrm{~g}, 0.125 \mathrm{~mol})$ was added. In a seperate flask, tert butyl hydroperoxide (70\% in water, $60 \mathrm{~mL}, 0.500 \mathrm{~mol})$ was mixed with dichloromethane $(200 \mathrm{~mL})$ and dried over $\mathrm{MgSO}_{4}$. This mixture was filtered and added directly to the stirring slurry of $\mathrm{SeO}_{2}$. The mixture was cooled to $0{ }^{\circ} \mathrm{C}$ and $\mathrm{AcOH}(0.025 \mathrm{~mol})$ was added. After most of the Selenium oxide had dissolved (approx. $30 \mathrm{~min}$ ), a solution of the divinylcyclohexanol 7 (as a mixture of diastereomers, $34.9 \mathrm{~g}, 0.250 \mathrm{~mol}$ ) was added. The reaction was allowed to stir for 2 days at room temperature until most of the starting material had been consumed. The reaction mixture was diluted with benzene $(100 \mathrm{~mL})$ and the dichloromethane was removed in vacuo. The residue was diluted again with Ethyl Acetate $(200 \mathrm{~mL})$, washed with $10 \% \mathrm{KOH}$ solution $\left(10 \% \mathrm{w} / \mathrm{w}\right.$ in $\mathrm{H}_{2} \mathrm{O}, 3$ x $50 \mathrm{~mL})$, Brine $(2 \times 50 \mathrm{~mL})$, and dried over $\mathrm{MgSO}_{4}$ and filtered. Silica $(\sim 50 \mathrm{~g})$ was added prior 
to removal of the volatiles. The dry powder was subjected to flash chromatography ( 0 to $30 \%$ EtOAc in 3 steps) to give the desired trans ring junction diol $\mathbf{8 b}$ as a light yellow oil. (15.3 $\mathrm{g}$, $34 \%$ over 2 steps). The minor diastereomer $8 \mathbf{a}$ was recovered by increasing the polarity to $40 \%$ EtOAc in Hexanes to give a thick yellow oil (9.25 g, 21\%).

\section{Data for 8b:}

${ }^{1}$ H NMR $\left(300 \mathrm{MHz}, \mathrm{CDCl}_{3}\right) \delta_{\mathrm{ppm}}=5.17(\mathrm{~s}, 1 \mathrm{H}), 5.06(\mathrm{~s}, 1 \mathrm{H}), 4.41(\mathrm{br} \mathrm{s}, 1 \mathrm{H}), 4.13(\mathrm{~m}, 2 \mathrm{H}), 2.87$ (br s, 1H), 2.41 (s, 1H), 2.34 (dd, J = 3.4, 12.9 Hz, 1H) 2.14-2.06 (m, 1H), 1.87-1.24 (m, 3H), 1.50-1.17 (m, $4 \mathrm{H}) .{ }^{13} \mathrm{C}$ NMR $\left(75 \mathrm{MHz}, \mathrm{CDCl}_{3}\right) \delta_{\mathrm{ppm}}=148.5$ (C QUAT.), $117.4\left(\mathrm{CH}_{2}\right), 88.5(\mathrm{C}$ QUAT.), 71.8 (C QUAT.), $67.6(\mathrm{CH}), 64.8\left(\mathrm{CH}_{2}\right), 52.4(\mathrm{CH}) 39.5\left(\mathrm{CH}_{2}\right) 26.0\left(\mathrm{CH}_{2}\right), 25.6\left(\mathrm{CH}_{2}\right)$,

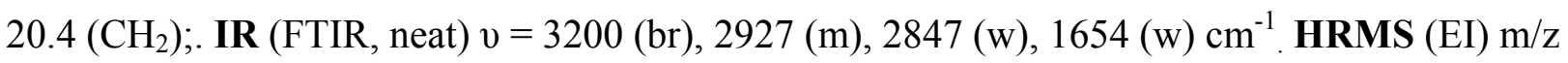
calc'd for $\mathrm{C}_{11} \mathrm{H}_{14} \mathrm{O}\left[\left(\mathrm{M}-\mathrm{H}_{2} \mathrm{O}\right)^{+}\right]$: 162.1045 , found: 162.1050

\section{Data for 8a:}

${ }^{1} \mathbf{H}$ NMR $\left(300 \mathrm{MHz}, \mathrm{CDCl}_{3}\right) \delta_{\mathrm{ppm}}=5.25(\mathrm{~s}, 1 \mathrm{H}), 5.19(\mathrm{~s}, 1 \mathrm{H}), 4.36(\mathrm{~s}, 1 \mathrm{H}), 4.18(\mathrm{~d}, \mathrm{~J}=12.4 \mathrm{~Hz}$, $1 \mathrm{H}), 4.02(\mathrm{~d}, \mathrm{~J}=12.4 \mathrm{~Hz}, 1 \mathrm{H}), 3.18(\mathrm{~s}, 1 \mathrm{H}), 2.49$ (s, 1H) 2.18-2.05 (m, 2H), 1.77-1.47 (m, 6H) 1.32-1.13 (m, 1H). ${ }^{13} \mathbf{C}$ NMR (75 MHz, $\left.\mathrm{CDCl}_{3}\right) \delta_{\mathrm{ppm}}=148.2$ (C QUAT.), $116.0\left(\mathrm{CH}_{2}\right), 85.2(\mathrm{C}$ QUAT.), 74.7 (CH), 72.0 (C QUAT.), $67.4\left(\mathrm{CH}_{2}\right), 51.7(\mathrm{CH}), 41.3\left(\mathrm{CH}_{2}\right), 30.0\left(\mathrm{CH}_{2}\right), 25.7\left(\mathrm{CH}_{2}\right)$ $23.7\left(\mathrm{CH}_{2}\right)$; IR (FTIR, neat) v = 3300 (br, m), 2934 (s), 2859 (m), 1651 (w), 1445 (w), 1124 (w), 1064 (m), $1004(\mathrm{w}), 906(\mathrm{w}) \mathrm{cm}^{-1}$.HRMS (EI) Expected for $\mathrm{C}_{11} \mathrm{H}_{16} \mathrm{O}_{2}\left[(\mathrm{M})^{+}\right]$: 180.11503, found: 180.11467

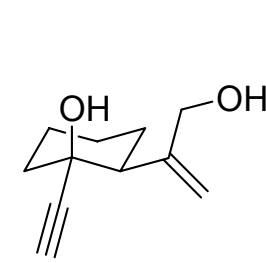

$8 \mathbf{b}$

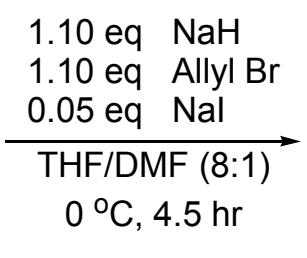

$65-77 \%$

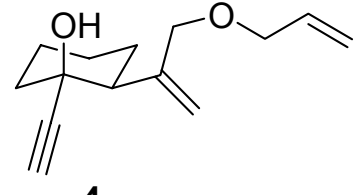

4

2-(1-Allyloxymethyl-vinyl)-1-ethynyl-cyclohexanol (4). A solution of diol 8b (15.3 g, $84.7 \mathrm{mmol})$ in a mixture of dry THF $(200 \mathrm{~mL})$ and DMF $(20 \mathrm{~mL})$ was cooled to $0{ }^{\circ} \mathrm{C}, \mathrm{NaI}(1.20$ $\mathrm{g}, 8.5 \mathrm{mmol})$ and freshly distilled allyl bromide $(9.18 \mathrm{~mL}, 101 \mathrm{mmol})$ was added. The mixture 
was allowed to stir at $0{ }^{\circ} \mathrm{C}$, and $\mathrm{NaH}(60 \% \mathrm{w} / \mathrm{w}, 101 \mathrm{mmol})$ was added in small portions. The orange mixture was stirred for 3 hours at $0{ }^{\circ} \mathrm{C}$, and quenched with $\mathrm{NH}_{4} \mathrm{Cl}_{(\mathrm{aq})}(40 \mathrm{~mL})$, extracted with diethyl ether $(4 \mathrm{x})$, dried over $\mathrm{MgSO}_{4}$ and concentrated. The crude was subjected to flash chromatography ( 0 to $10 \% \mathrm{Et}_{2} \mathrm{O}$ in Hexanes) to give the allyl ether 4 as a pale yellow oil (14.2 $\mathrm{g}$, $77 \%)$

${ }^{1} \mathbf{H}$ NMR $\left(\mathrm{CDCl}_{3}, 300 \mathrm{MHz}\right) \delta_{\mathrm{ppm}}=5.92-5.79(\mathrm{~m}, 1 \mathrm{H}), 5.24(\mathrm{~d}, \mathrm{~J}=17.2 \mathrm{~Hz}, 1 \mathrm{H}), 5.17-5.13(\mathrm{~m}$, $3 \mathrm{H}), 4.48(\mathrm{~s}, 1 \mathrm{H}), 4.11-4.06(\mathrm{~m}, 2 \mathrm{H}), 3.91-3.78(\mathrm{~m}, 2 \mathrm{H}), 2.35-2.27(\mathrm{~m}, 2 \mathrm{H}), 2.10-2.04(\mathrm{~m}, 1 \mathrm{H})$, $1.83-1.50(\mathrm{~m}, 4 \mathrm{H}), 1.48-1.13(\mathrm{~m}, 3 \mathrm{H}) .{ }^{13} \mathbf{C} \mathbf{N M R}\left(\mathrm{CDCl}_{3}, 75 \mathrm{MHz}\right) \delta_{\mathrm{ppm}}=114.8$ (C QUAT.) $133.6(\mathrm{CH}), 119.6\left(\mathrm{CH}_{2}\right), 117.7\left(\mathrm{CH}_{2}\right), 88.5$ (C QUAT.), $71.5\left(\mathrm{CH}_{2}\right), 71.2$ (C QUAT.), 70.4 $\left(\mathrm{CH}_{2}\right), 67.3(\mathrm{CH}), 52.9(\mathrm{CH}), 39.4\left(\mathrm{CH}_{2}\right), 25.7\left(2 \times \mathrm{CH}_{2}\right), 20.3\left(\mathrm{CH}_{2}\right)$. IR (FTIR, neat) v $=3306$ (s), 3300 (m), 3079 (m), 2936 (s), 2857 (s), 1644 (m), 1446 (m), 1351 (m), 1144 (m), 1070 (s), 977 (s), 922 (s), 648 (m). HRMS (EI) Expected for $\mathrm{C}_{11} \mathrm{H}_{14} \mathrm{O}\left[\left(\mathrm{M}-\mathrm{C}_{3} \mathrm{H}_{6} \mathrm{O}\right)^{+}\right]: 162.1045$, found 162.1061 .

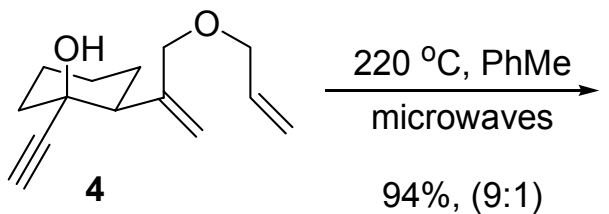

$94 \%,(9: 1)$

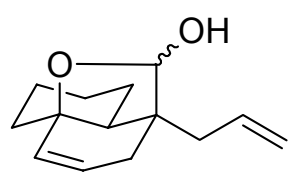

5

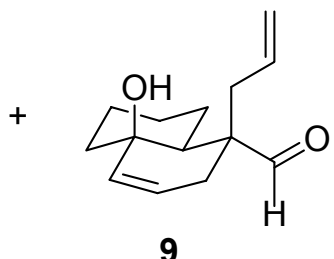

9

7-Allyl-11-oxa-tricyclo[5.3.2.0 $\left.{ }^{1,6}\right]$ dodec-9-en-12-ol (5) and 1-Allyl-4a-hydroxy1,2,4a,5,6,7,8,8a-octahydro-naphthalene-1-carbaldehyde (9). A dry microwave cell fitted with two Carboflons ${ }^{\mathrm{TM}}$ (a polymer based microwave absorbant) was charged with a solution of the allyl ether $4(2.00 \mathrm{~g})$ and $\mathrm{Et}_{3} \mathrm{~N}(1 \mathrm{~mL})$ in Toluene $(17 \mathrm{~mL})$. The mixture was sealed in the microwave apparatus and heated at $220{ }^{\circ} \mathrm{C}$ for 45 minutes. After cooling, the volatiles were removed, and the residue subjected to flash chromatography. (0 to 30\% EtOAc in Hexanes) to give the lactol 5 as a mixture of diastereomers at the anomeric position $(1.71 \mathrm{~g}, 85 \%)$ as a pale yellow solid and the aldehyde $\mathbf{9}(173 \mathrm{mg}, 9 \%)$ as an amorphous yellow semisolid.

\section{Data for 5:}


${ }^{1}$ H NMR $\left(300 \mathrm{MHz}, \mathrm{CDCl}_{3}\right) \delta_{\mathrm{ppm}}=5.83-5.51(\mathrm{~m}, 3 \mathrm{H}) 5.24(\mathrm{~d}, \mathrm{~J}=6.9 \mathrm{~Hz}, 0.55 \mathrm{H})$, 5.07-4.96 (m, $2.45 \mathrm{H}) 4.97(\mathrm{~d}, \mathrm{~J}=4.3 \mathrm{~Hz}, 0.45 \mathrm{H}) 4.07(\mathrm{~d}, \mathrm{~J}=4.3 \mathrm{~Hz}, 0.55 \mathrm{H}) 2.40-2.11(\mathrm{~m}, 3 \mathrm{H}) 1.98-1.85(\mathrm{~m}$, 2H) $1.73-1.23(\mathrm{~m}, 8 \mathrm{H}) .{ }^{\mathbf{1 3}} \mathbf{C}$ NMR $\left(75 \mathrm{MHz}, \mathrm{CDCl}_{3}\right) \delta_{\mathrm{ppm}}=137.2,136.6,135.4,134.7,128.1$, 126.5, 117.4, 117.3, 105.1, 103.8, 78.5, 49.3, 49.1, 48.8, 45.9, 38.7, 37.3, 35.3, 33.3, 32.6, 32.4, 29.6, 25.0, 24.7, 23.3, 23.2, 21.0, 20.9.IR (FTIR, neat) v = 3379 (br, s), 3074, 3025, 2930, 2855, 1822, 1727, 1640, 1143, $1100 \mathrm{~cm}^{-1}$. HRMS (EI) Expected for C QUAT. $\mathrm{H}_{18} \mathrm{O}\left[\left(\mathrm{M}-\mathrm{H}_{2} \mathrm{O}\right)^{+}\right]$: 202.1358 , found 202.1354 .

\section{Data for 9:}

${ }^{1} \mathbf{H}$ NMR $\left(500 \mathrm{MHz}, \mathrm{C}_{6} \mathrm{D}_{6}\right) \delta_{\mathrm{ppm}}=9.28(\mathrm{~s}, 1 \mathrm{H})$ 5.82-5.73 (m, 1H), 5.43-5.37 (m, 2H), 5.03-4.95 (m, 2H) $2.77(\mathrm{dd}, \mathrm{J}=8.6,14 \mathrm{~Hz}, 1 \mathrm{H}) 2.65(\mathrm{ddd}, \mathrm{J}=6.1,14,1.3 \mathrm{~Hz}, 1 \mathrm{H}) 2.12(\mathrm{~d}, \mathrm{~J}=17 \mathrm{~Hz}, 1 \mathrm{H})$ $1.86(\mathrm{dd}, \mathrm{J}=17,2.8 \mathrm{~Hz}, 1 \mathrm{H})$ 1.80-1.45 (m, 4H), 1.40-0.56 (m, 6H). ${ }^{13} \mathbf{C}$ NMR $\left(125 \mathrm{MHz}, \mathrm{C}_{6} \mathrm{D}_{6}\right)$ $\delta_{\mathrm{ppm}}=205.5(\mathrm{CH}), 136.5(\mathrm{CH}), 133.6(\mathrm{CH}), 124.9(\mathrm{CH}), 117.9\left(\mathrm{CH}_{2}\right), 67.3(\mathrm{C}$ QUAT. $), 50.5(\mathrm{C}$ QUAT.) $46.3(\mathrm{CH}), 40.6\left(\mathrm{CH}_{2}\right), 33.1\left(\mathrm{CH}_{2}\right), 29.6\left(\mathrm{CH}_{2}\right), 26.9\left(\mathrm{CH}_{2}\right), 22.4\left(\mathrm{CH}_{2}\right), 21.3\left(\mathrm{CH}_{2}\right)$. IR $\left(\right.$ FTIR, neat) $v=3510(\mathrm{~s}), 3074.2,3019,2930,2857,2704,1720(\mathrm{~s}), 1436,958,915 \mathrm{~cm}^{-1}$.

HRMS (EI) Expected for $\mathrm{C}_{14} \mathrm{H}_{20} \mathrm{O}_{2}\left[(\mathrm{M})^{+}\right]$: 220.14633, Found 220.14522 (3.2\%).

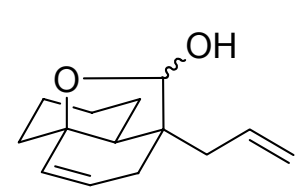

5

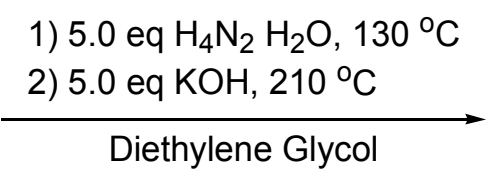

$80-88 \%$

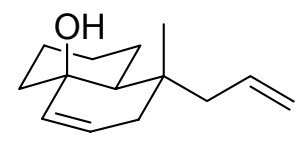

10

8-Allyl-8-methyl-1,3,4,7,8,8a-hexahydro-2H-naphthalen-4a-ol (10). A solution of the lactol 5 (1.62 g, $7.36 \mathrm{mmol})$ and diethylene glycol $(37 \mathrm{~mL})$ in a $250 \mathrm{~mL}$ roundbottom flask was degassed by stirring under high vacuum for 45 minutes. Hydrazine hydrate $(36.8 \mathrm{mmol})$ was added, and the flask was fitted with a reflux condenser. The atmosphere over the solution was replaced with argon and the mixture was heated to $130{ }^{\circ} \mathrm{C}$ for $1 \mathrm{~h}$ The reaction was cooled back to room temperature and $\mathrm{KOH}(37 \mathrm{mmol})$ was added in one portion. The reaction was heated to 210 ${ }^{\circ} \mathrm{C}$ for 3 hours and allowed to cool. Water $(100 \mathrm{~mL})$ and $\mathrm{Et}_{2} \mathrm{O}(30 \mathrm{~mL})$ was added. The biphasic mixture was allowed to stir 30 minutes. The organic phase was extracted with ether $(5 \times 30 \mathrm{~mL})$, 
dried over $\mathrm{MgSO}_{4}$ and concentrated. The crude oil was subjected to flash chromatography (5\% $\mathrm{Et}_{2} \mathrm{O}$ in Hexanes) to give $\mathbf{1 0}$ as a colourless oil (1.21 g, 80\%).

${ }^{1} \mathbf{H}$ NMR $\left(\mathrm{CDCl}_{3}, 500 \mathrm{MHz}\right) \delta_{\mathrm{ppm}}=5.80-5.70(\mathrm{~m}, 1 \mathrm{H}), 5.65(\mathrm{ddd}, \mathrm{J}=2.2,5.4,9.9 \mathrm{~Hz}, 1 \mathrm{H}), 5.54$ (br d, J = 9.9 Hz, 1H), 5.07-4.98 (m, 2H), 2.13-2.02 (m, 2H), 1.95-1.87 (m, 1H), 1.84-1.77 (m, $1 \mathrm{H}), 1.73-1.47(\mathrm{~m}, 6 \mathrm{H}), 1.35-1.17(\mathrm{~m}, 4 \mathrm{H}), 0.95(\mathrm{~s}, 3 \mathrm{H}) .{ }^{13} \mathbf{C ~ N M R}\left(\mathrm{CDCl}_{3}, 125 \mathrm{MHz}\right) \delta_{\mathrm{ppm}}=$ $134.8(\mathrm{CH}), 133.2(\mathrm{CH}), 127.4(\mathrm{CH}), 117.6\left(\mathrm{CH}_{2}\right), 69.1$ (C QUAT.) $48.7\left(\mathrm{CH}_{2}\right), 45.9(\mathrm{CH}), 40.1$ (CH), $38.0(\mathrm{CH}), 34.0$ (C QUAT.), $26.7(\mathrm{CH}), 21.3\left(\mathrm{CH}_{2}\right), 21.3\left(\mathrm{CH}_{2}\right), 20.2\left(\mathrm{CH}_{3}\right)$. IR (FTIR, neat) $v=3485$ (br s), 3073, 3013, 2931 (s), 1637, 1445, 1380, 1142, 995, 939, $913 \mathrm{~cm}^{-1}$. HRMS (EI) Expected for $\mathrm{C}_{14} \mathrm{H}_{20}\left[\left(\mathrm{M}-\mathrm{H}_{2} \mathrm{O}\right)^{+}\right]$: 188.15650 , found $188.15492(3.4 \%)$

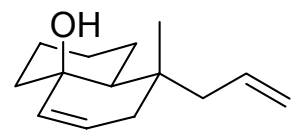

10

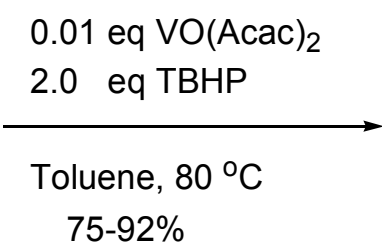

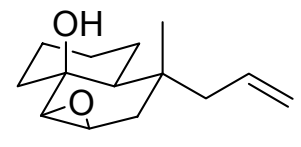

11

3-Allyl-3-methyl-octahydro-1-oxa-cyclopropa[a]naphthalen-7a-ol (11). A solution of the olefin 10 (1.52 g, $7.36 \mathrm{mmol})$ in toluene $(75 \mathrm{~mL})$ was stirred at room temperature, and $\mathrm{VO}(\text { Acac })_{2}$ (75 mg, $0.29 \mathrm{mmol}, 0.04$ equiv) was added. The mixture was sonicated briefly and allowed to stir for $1 \mathrm{~h}$ until the catalyst had dissolved (to give a dark green solution). tert-butyl hydroperoxide (5-6M in Pentane, 2.0 equiv) was added.to give a deep purple mixture. The mixture was heated to $60-70{ }^{\circ} \mathrm{C}$ for $3 \mathrm{~h}$ and allowed to cool. The reaction was diluted with ether, and $\mathrm{NH}_{4} \mathrm{Cl}_{(\mathrm{aq})}(10 \mathrm{~mL})$ was added. The organic phases were extracted with EtOAc $(3 \times 20 \mathrm{~mL})$, dried over $\mathrm{MgSO}_{4}$ and concentrated. The residue was subjected to flash chromatography (15\% EtOAc in Hexanes) to give 11 as a light yellow oil (1.48 g, 91\%). ${ }^{1} \mathbf{H} \mathbf{~ N M R}\left(500 \mathrm{MHz}, \mathrm{CDCl}_{3}\right)$ $\delta_{\text {ppm }}=5.73-5.64(\mathrm{~m}, 1 \mathrm{H}), 5.09-4.87(\mathrm{~m}, 2 \mathrm{H}), 3.41(\mathrm{ddd}, \mathrm{J}=3.9 .2 .1,2.1 \mathrm{~Hz}, 1 \mathrm{H}), 3.0(\mathrm{~d}, \mathrm{~J}=3.9$ $\mathrm{Hz}, 1 \mathrm{H}), 2.05$ (br s, 1H), 1.95 (dd, J = 13.8, $6.70 \mathrm{~Hz}, 1 \mathrm{H}), 1.90-1.65$ (m, 7H), 1.60-1.38 (m, 5H), $0.93(\mathrm{~s}, 3 \mathrm{H}) .{ }^{13} \mathrm{C}$ NMR $\left(125 \mathrm{MHz}, \mathrm{CDCl}_{3}\right) \delta_{\mathrm{ppm}}=134.3(\mathrm{CH}), 118.3\left(\mathrm{CH}_{2}\right), 68.6$ (C QUAT.), 59.4 (C QUAT.), $59.3(\mathrm{CH}), 56.6(\mathrm{CH}), 48.7(\mathrm{CH}), 47.4\left(\mathrm{CH}_{2}\right), 39.6\left(\mathrm{CH}_{2}\right), 34.9\left(\mathrm{CH}_{2}\right), 23.4$ (CH), 21.5 (2 x CH$), 20.2\left(\mathrm{CH}_{3}\right)$. IR (FTIR, neat) v = 3590 (br), 3073, 2933, 2850 1638, 1446, $973 \mathrm{~cm}^{-1}$. HRMS (EI) Expected for $\mathrm{C}_{14} \mathrm{H}_{22} \mathrm{O}_{2}\left[(\mathrm{M})^{+}\right]: 222.16198$, found: 222.16007 (1.0\%) 


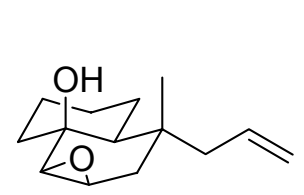

11

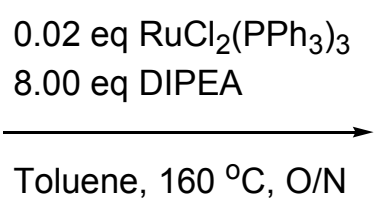

Toluene, $160^{\circ} \mathrm{C}, \mathrm{O} / \mathrm{N}$

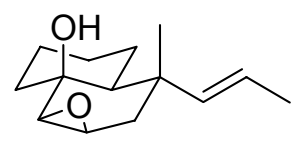

$12(83 \%)$

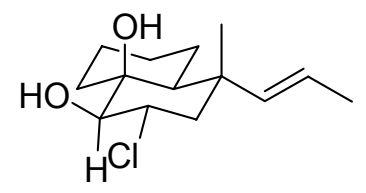

13

3-Methyl-3-propenyl-octahydro-1-oxa-cyclopropa[a]naphthalen-7a-ol (12) and 2Chloro-4-methyl-4-propenyl-octahydro-naphthalene-1,8a-diol (13). A solution of the epoxide 11 (1.040 g, $4.73 \mathrm{mmol}$ ) was stirred in toluene $(47 \mathrm{~mL})$ under argon in a flask fitted with a reflux condeser at room temperature. Hunig's Base (DIPEA, $6.6 \mathrm{~mL}, 8 \mathrm{eq}$ ) was added, followed by $\mathrm{RuCl}_{2}\left(\mathrm{PPh}_{3}\right)_{3}(90 \mathrm{mg}, 0.02 \mathrm{eq})$. The mixture was heated to $130{ }^{\circ} \mathrm{C}$ for 18 hours, and was followed by GC. The reaction was cooled to room temperature and the solvent removed under reduced pressure. The residue was subjected to column chromatography (0 to $15 \%$ gradient EtOAc in hexanes) to give olefin 12 (862 mg, 83\%) as a clear oil. Compound $\mathbf{1 3}$ was isolated in an analogous procedure utilizing 0.1 eq of $\mathrm{RuCl}_{2}\left(\mathrm{PPh}_{3}\right)_{2}$, and was obtained as a colourless solid $(10 \%)$.

\section{Data for 12:}

${ }^{1} \mathbf{H}$ NMR $\left(\mathrm{CDCl}_{3}, 300 \mathrm{MHz}\right) \delta_{\mathrm{ppm}}=0.83-0.90(\mathrm{~m}, 2 \mathrm{H}) 1.00(\mathrm{~s}, 3 \mathrm{H}) 1.14-1.25(\mathrm{~m}, 1 \mathrm{H}) 1.35-1.50$ $(\mathrm{m}, 3 \mathrm{H}) 1.58-1.59(\mathrm{~m}, 1 \mathrm{H}) 1.62(\mathrm{dd}, 3 \mathrm{H}, \mathrm{J}=6.4,1.6 \mathrm{~Hz}) 1.67-1.84(\mathrm{~m}, 4 \mathrm{H}) 1.93(\mathrm{dd}, 1 \mathrm{H}, \mathrm{J}=15.7$, $1.6 \mathrm{~Hz}) 2.04(\mathrm{~d}, 1 \mathrm{H}, \mathrm{J}=2.3 \mathrm{~Hz}) 2.98(\mathrm{~d}, 1 \mathrm{H}, \mathrm{J}=3.9 \mathrm{~Hz}) 3.38(\mathrm{ddd}, \mathrm{J}=3.9,3.8,1.6 \mathrm{~Hz}, 1 \mathrm{H}) 5.10$ $(\mathrm{dd}, 1 \mathrm{H}, \mathrm{J}=15.5,1.6 \mathrm{~Hz}) 5.26(\mathrm{dt}, 1 \mathrm{H}, \mathrm{J}=15.5,6.4 \mathrm{~Hz}) .{ }^{13} \mathbf{C} \mathbf{N M R}\left(\mathrm{CDCl}_{3}, 75 \mathrm{MHz}\right) \delta_{\mathrm{ppm}}=142.5$ $(\mathrm{CH}), 121.9(\mathrm{CH}), 68.6(\mathrm{C}$ QUAT. $), 59.3(\mathrm{CH}), 56.4(\mathrm{CH}), 50.4(\mathrm{CH}), 39.6\left(\mathrm{CH}_{2}\right), 37.9(\mathrm{C}$ QUAT.), $37.0\left(\mathrm{CH}_{2}\right), 26.9\left(\mathrm{CH}_{2}\right), 21.6\left(\mathrm{CH}_{2}\right), 21.3\left(\mathrm{CH}_{2}\right), 20.0\left(\mathrm{CH}_{3}\right), 18.0\left(\mathrm{CH}_{3}\right)$. IR (FTIR, Neat) $v=3358$ (br), 2934, 2851, 1446, $972 \mathrm{~cm}^{-1}$. HRMS (EI) Expected for $\mathrm{C}_{14} \mathrm{H}_{22} \mathrm{O}_{2}\left[(\mathrm{M})^{+}\right]$: 222.16198, Found: $222.16022(2.00 \%)$

\section{Data for 13:}

${ }^{1} \mathbf{H}$ NMR $\left(\mathrm{CDCl}_{3}, 500 \mathrm{MHz}\right) \delta_{\mathrm{ppm}}=5.41-5.32(\mathrm{~m}, 1 \mathrm{H}), 5.18(\mathrm{dd}, \mathrm{J}=1.4,15.5 \mathrm{~Hz}, 1 \mathrm{H}), 4.27$ $(\mathrm{ddd}, \mathrm{J}=4.2,9.7,13.8 \mathrm{~Hz}, 1 \mathrm{H}), 3.25(\mathrm{dd}, \mathrm{J}=9.7,1.6 \mathrm{~Hz}, 1 \mathrm{H}), 2.62(\mathrm{~d}, \mathrm{~J}=2.2 \mathrm{~Hz}, 1 \mathrm{H}), 2.02(\mathrm{~d}, \mathrm{~J}$ $=13.8 \mathrm{~Hz}, 1 \mathrm{H}), 1.92-1.85(\mathrm{~m}, 2 \mathrm{H}), 1.67-1.63(\mathrm{~m}, 2 \mathrm{H}), 1.63(\mathrm{dd}, \mathrm{J}=1.4,6.4 \mathrm{~Hz}, 3 \mathrm{H}), 1.62-1.39$ $(\mathrm{m}, 5 \mathrm{H}), 1.30-1.12(\mathrm{~m}, 2 \mathrm{H}), 1.11(\mathrm{~s}, 3 \mathrm{H}) .{ }^{1} \mathbf{C} \mathbf{N M R}\left(\mathrm{CDCl}_{3}, 125 \mathrm{MHz}\right) \delta_{\mathrm{ppm}}=141.4(\mathrm{CH}), 122.4$ (CH), 80.7 (CH), 74.2 (C QUAT.), $62.3(\mathrm{CH}) 49.5(\mathrm{CH}), 48.1\left(\mathrm{CH}_{2}\right), 41.0$ (C QUAT.), 37.5 
$\left(\mathrm{CH}_{2}\right), 26.4\left(\mathrm{CH}_{2}\right) 21.9\left(\mathrm{CH}_{2}\right), 21 .\left(\mathrm{CH}_{2}\right), 18.5\left(\mathrm{CH}_{3}\right), 18.1\left(\mathrm{CH}_{3}\right)$. HRMS (EI) Expected for $\mathrm{C}_{14} \mathrm{H}_{23} \mathrm{O}_{2} \mathrm{Cl}\left[(\mathrm{M})^{+}\right]: 258.13866$, found: 258.14030 (3.85\%), $259.14316(0.58 \%)$.

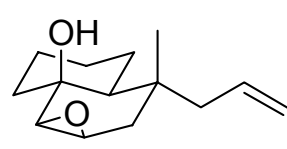

11

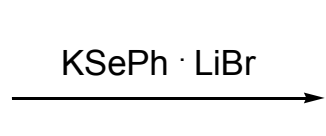

THF, RT

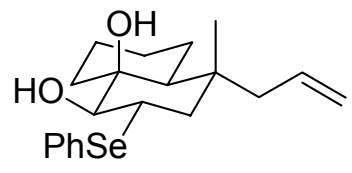

14

4-Allyl-4-methyl-2-phenylselanyl-octahydro-naphthalene-1,8a-diol (14). See protocol for 20, below. Starting material 11 (29 mg, $13.2 \mu \mathrm{mol})$. Product (14) as thick yellow oil (31 mg, $62 \%)$.

${ }^{1} \mathbf{H}$ NMR $\left(\mathrm{CDCl}_{3}, 500 \mathrm{MHz}\right) \delta_{\mathrm{ppm}}=7.58-7.24(\mathrm{~m}, 5 \mathrm{H}), 5.67-5.59(\mathrm{~m}, 1 \mathrm{H}), 4.97(\mathrm{~d}, \mathrm{~J}=10.1 \mathrm{~Hz}$, 1H), $4.91(\mathrm{~d}, \mathrm{~J}=17.0 \mathrm{~Hz}, 1 \mathrm{H}), 2.95-2.90(\mathrm{~m}, 1 \mathrm{H}), 2.90$ (d, J = 10.6 Hz, 1H), 1.99 (br d, J = 17.0 Hz), 1.90 (d, J = 7.4 Hz, 2H), 1.81 (dd, J = 13.4, 2.9 Hz, 1H), 1.70 (br d, J = 13.0 Hz, 1H), 1.67$1.40(\mathrm{~m}, 6 \mathrm{H}), 1.13-1.04(\mathrm{~m}, 2 \mathrm{H}), 1.01(\mathrm{~s}, 3 \mathrm{H}), 1.00-0.93(\mathrm{~m}, 1 \mathrm{H}) .{ }^{13} \mathbf{C} \mathbf{N M R}\left(\mathrm{CDCl}_{3}, 125 \mathrm{MHz}\right)$ $\delta_{\text {ppm }}=136.0(2 \times \mathrm{CH}), 134.2(\mathrm{CH}), 128.9(2 \times \mathrm{CH}), 128.2(\mathrm{CH}), 125.5(\mathrm{C}$ QUAT. $), 117.5\left(\mathrm{CH}_{2}\right)$, 73.7 (C QUAT.), $47.5(\mathrm{CH}), 46.7\left(\mathrm{CH}_{2}\right), 46.0(\mathrm{CH}), 44.0\left(\mathrm{CH}_{2}\right), 38.1$ (C QUAT.), $37.7\left(\mathrm{CH}_{2}\right)$, $26.3\left(\mathrm{CH}_{2}\right), 21.3\left(2 \times \mathrm{CH}_{2}\right), 21.0\left(\mathrm{CH}_{3}, \mathrm{CH}_{2}\right)$. IR (FTIR, neat) $v=3492$ (br s), 3071 (m), 2934 (s), 2856 (s), 1477, 1438, 1380 (m), $980 \mathrm{~cm}^{-1}$. HRMS (EI) Expected for $\mathrm{C}_{20} \mathrm{H}_{28} \mathrm{O}_{2} \mathrm{Se}\left[(\mathrm{M})^{+}\right]$: 380.12545 , found: $380.12681(52.9 \%)$
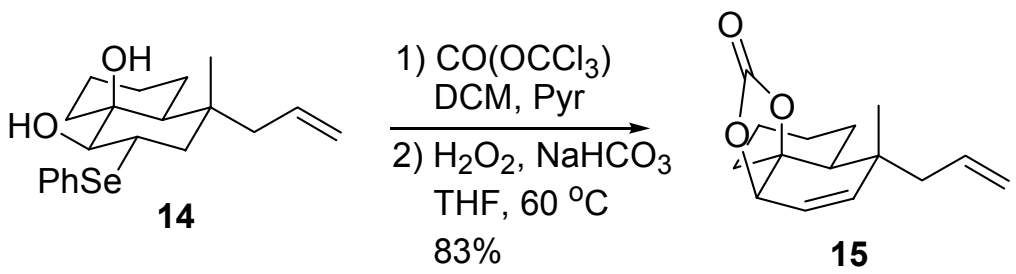

\section{6-Allyl-6-methyl-6,6a,7,8,9,10-hexahydro-3aH-naphtho[1,8a-d][1,3]dioxol-2-one (15).} A solution of selenide $14(44 \mathrm{mg}, 116 \mu \mathrm{mol})$ in DCM $(3 \mathrm{~mL})$ and Pyridine $(100 \mu \mathrm{L})$ was stirred at $0{ }^{\circ} \mathrm{C}$. Triphosgene (35 mg, 1 equiv) was added as a dichloromethane solution ( $\left.1 \mathrm{~mL}\right)$. The reaction was allowed to warm to room temperature with stirring over $2 \mathrm{~h}$. The mixture was

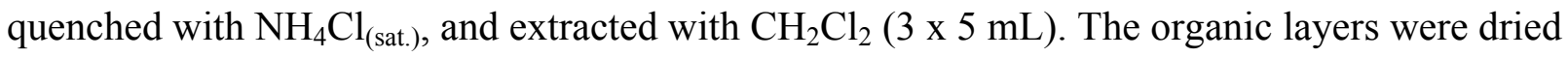
over $\mathrm{MgSO}_{4}$ and concentrated. The crude carbonate was redissolved in THF (5 mL) and stirred at 
room temperature. $\mathrm{NaHCO}_{3}(10 \mathrm{mg})$ was added, followed by $\mathrm{H}_{2} \mathrm{O}_{2}\left(30 \%\right.$ in $\mathrm{H}_{2} \mathrm{O}, 2$ equiv). The reaction was heated to $60{ }^{\circ} \mathrm{C}$ for $1 \mathrm{~h}$, and allowed to cool. The mixture was partitioned between Ether $(10 \mathrm{~mL})$ and saturated ammonium chloride $(5 \mathrm{~mL})$. Extraction of the organic phase and drying over $\mathrm{MgSO}_{4}$, followed by concentration gave a yellow oil that was subjected to flash chromatography (15\% EtOAc in Hexanes) to give 15 as a clear oil (24.2 $\mathrm{mg}, 83 \%)$.

${ }^{1} \mathbf{H}$ NMR $\left(\mathrm{CDCl}_{3}, 500 \mathrm{MHz}\right) \delta_{\mathrm{ppm}}=5.87(\mathrm{~d}, \mathrm{~J}=10.4 \mathrm{~Hz}, 1 \mathrm{H}), 5.61(\mathrm{dd}, \mathrm{J}=3.1,10.4 \mathrm{~Hz}, 1 \mathrm{H})$, 5.88-5.86 (m, 1H), $5.14(\mathrm{~d}, \mathrm{~J}=16.2 \mathrm{~Hz}, 1 \mathrm{H}), 5.04(\mathrm{~d}, \mathrm{~J}=10.2 \mathrm{~Hz}, 1 \mathrm{H}), 4.98(\mathrm{~d}, \mathrm{~J}=17.0 \mathrm{~Hz}, 1 \mathrm{H})$ $4.52(\mathrm{~d}, \mathrm{~J}=3.1 \mathrm{~Hz}, 1 \mathrm{H}), 2.20-2.04(\mathrm{~m}, 3 \mathrm{H}), 1.87-1.82(\mathrm{~m}, 1 \mathrm{H}), 1.76-1.52(\mathrm{~m}, 5 \mathrm{H}), 1.45-1.21(\mathrm{~m}$, 2H) 0.97 (s, 3H). ${ }^{13} \mathbf{C} \mathbf{N M R}\left(\mathrm{CDCl}_{3}, 125 \mathrm{MHz}\right) \delta_{\mathrm{ppm}}=154.1$ (C QUAT.), $143.2(\mathrm{CH}), 134.1$ $(\mathrm{CH}), 119.3(\mathrm{CH}), 118.3\left(\mathrm{CH}_{2}\right), 83.5$ (C QUAT.), $77.8(\mathrm{CH}), 45.2\left(\mathrm{CH}_{2}\right), 42.0(\mathrm{CH}), 37.4\left(\mathrm{CH}_{2}\right)$, 37.0 (C QUAT.), $25.5\left(\mathrm{CH}_{2}\right), 23.7\left(\mathrm{CH}_{3}\right), 22.4\left(\mathrm{CH}_{2}\right), 21.3\left(\mathrm{CH}_{2}\right)$. IR (FTIR, Neat) v = 2939, 2866, 1797 (s), 1205, 1028, $1010 \mathrm{~cm}^{-1}$. HRMS (EI) Expected for $\mathrm{C}_{12} \mathrm{H}_{15} \mathrm{O}_{3}$ [(M-Allyl) $\left.{ }^{+}\right]$: 207.10212, Found: 207.1002 (71.0\%).

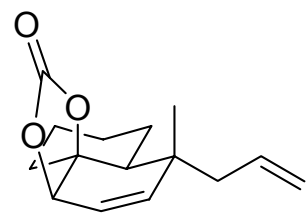

15

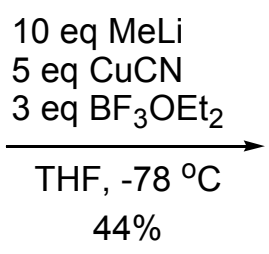

$44 \%$

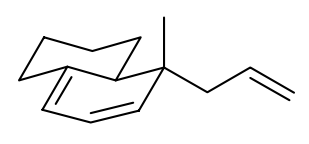

16

5-Allyl-5-methyl-1,2,3,4,4a,5-hexahydro-naphthalene (16). A slurry of CuCN (51 mg, 5 eq) in THF ( $5 \mathrm{~mL})$ was cooled to $-30{ }^{\circ} \mathrm{C}$ and MeLi (1.6M in THF, $\left.706 \mathrm{uL}, 10 \mathrm{eq}\right)$ was added. After the solution became clear and colourless $(5 \mathrm{~min})$ the reaction was cooled to $-78^{\circ} \mathrm{C}$ and $\mathrm{BF}_{3^{-}}$ Etherate was added ( $15 \mathrm{uL}, 3 \mathrm{eq})$ was added, followed by the carbonate $\mathbf{1 5}(28 \mathrm{mg})$ as a THF solution $(1 \mathrm{~mL})$. The reaction was allowed to stir $3 \mathrm{~h}$ at $-78^{\circ} \mathrm{C}$, with monitoring by TLC. Once complete, the reaction was quenched with $\mathrm{NH}_{4} \mathrm{Cl}_{(\text {sat })}: \mathrm{NH}_{4} \mathrm{OH}(9: 1)$, and extracted with ether. The residue was subjected to flash chromatography (100\% Hexanes) to give $\mathbf{1 6}$ as a colourless oil (9.0 $\mathrm{mg}, 44 \%)$

${ }^{1} \mathbf{H}$ NMR $\left(500 \mathrm{MHz}, \mathrm{CDCl}_{3}\right) \delta_{\mathrm{ppm}}=5.82-5.78(\mathrm{~m}, 1 \mathrm{H}), 5.70(\mathrm{dd}, \mathrm{J}=9.5,5.4 \mathrm{~Hz}, 1 \mathrm{H}), \quad 5.94(\mathrm{~d}, \mathrm{~J}$ $=5.1 \mathrm{~Hz}, 1 \mathrm{H}), 5.27(\mathrm{~d}, 9.6 \mathrm{~Hz}, 1 \mathrm{H}), 5.01-4.94(\mathrm{~m}, 2 \mathrm{H}), 2.28-2.25(\mathrm{~m}, 1 \mathrm{H}), 2.20(\mathrm{dd}, \mathrm{J}=7.4,13.5$ $\mathrm{Hz}, 1 \mathrm{H}) 2.05-1.92(\mathrm{~m}, 3 \mathrm{H}) 1.82-1.55(\mathrm{~m}, 3 \mathrm{H}) 1.40-1.22(\mathrm{~m}, 3 \mathrm{H}) 0.90(\mathrm{~s}, 3 \mathrm{H}) .{ }^{13} \mathbf{C}$ NMR (125 
$\left.\mathrm{MHz}, \mathrm{CDCl}_{3}\right) \delta_{\mathrm{ppm}}=143.5\left(\mathrm{C}\right.$ QUAT.), $135.74(\mathrm{CH}), 133.5(\mathrm{CH}), 121.6(\mathrm{CH}), 116.7\left(\mathrm{CH}_{2}\right)$, 114.9 (CH), $46.59(\mathrm{CH}), 45.9\left(\mathrm{CH}_{2}\right), 37.0(\mathrm{C}$ QUAT. $), 35.6\left(\mathrm{CH}_{2}\right), 29.4\left(\mathrm{CH}_{2}\right), 27.8\left(\mathrm{CH}_{2}\right), 26.8$ $\left(\mathrm{CH}_{2}\right), 21.8\left(\mathrm{CH}_{3}\right)$. IR (FTIR, Neat) $v=3072,2929,914 \mathrm{~cm}^{-1}$. HRMS (EI) Expected for $\mathrm{C}_{14} \mathrm{H}_{20}$ $\left[(\mathrm{M})^{+}\right]: 188.1565$, found: 188.1544

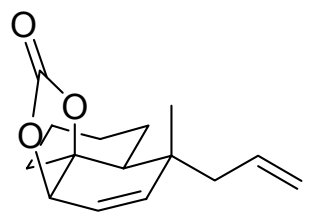

15

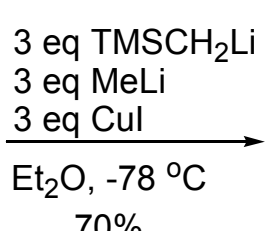

$70 \%$

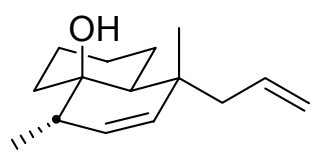

17

8-Allyl-5,8-dimethyl-1,3,4,5,8,8a-hexahydro-2H-naphthalen-4a-ol (17). A solution of $\mathrm{CuI}(483 \mathrm{mg}, 3 \mathrm{eq})$ in $\mathrm{Et}_{2} \mathrm{O}(8 \mathrm{~mL})$ was cooled to $-78^{\circ} \mathrm{C}$ and trimethylsilylmethyllithium $(240 \mathrm{mg}$, $3 \mathrm{eq})$ was added as an ether solution $(2 \mathrm{~mL})$, followed by Methyllithium (1.6M in Ether, $3 \mathrm{eq})$. The cuprate was annealed at $-30^{\circ} \mathrm{C}$ briefly and recooled to $-78^{\circ}$, and the carbonate 15 (199 mg, 1 eq) was added via canula and rinse ( $2 \mathrm{~mL}$ THF). The rx was stirred for $1 \mathrm{~h}$, while allowing to warm to $-20^{\circ} \mathrm{C}$, followed by quench with $\mathrm{NH}_{4} \mathrm{Cl}_{\text {(sat.) }}$ and workup as usual. Flash chromatography ( 0 to $10 \%$ EtOAc in Hex.) gave 17 as a light yellow oil (131 mg, 70\%)

${ }^{1} \mathbf{H}$ NMR $\left(\mathrm{CDCl}_{3}, 500 \mathrm{MHz}\right) \delta_{\mathrm{ppm}}=5.70-5.63(\mathrm{dddd}, 1 \mathrm{H}), 5.61(\mathrm{dd}, \mathrm{J}=10.2,5.5 \mathrm{~Hz}, 1 \mathrm{H}), 5.36$ (d, J = 10.2 Hz, 1H) 5.01-4.93 (m, 2H) 2.05-1.92 (m, 3H), 1.75-1.38 (m, 10H), $1.00(\mathrm{~s}, 3 \mathrm{H}), 0.89$ $(\mathrm{d}, \mathrm{J}=7.2 \mathrm{~Hz}, 3 \mathrm{H}) .{ }^{13} \mathbf{C} \mathbf{~ N M R}\left(\mathrm{CDCl}_{3}, 125 \mathrm{MHz}\right) \delta_{\mathrm{ppm}}=135.9(\mathrm{CH}), 135.5(\mathrm{CH}), 128.6(\mathrm{CH})$, 117.0 $\left(\mathrm{CH}_{2}\right), 73.4$ (C QUAT.), $46.2\left(\mathrm{CH}_{2}\right), 44.4(\mathrm{CH}), 42.2(\mathrm{CH}), 39.5(\mathrm{CH}), 37.9\left(\mathrm{CH}_{2}\right), 26.5$ $\left(\mathrm{CH}_{2}\right), 24.5\left(\mathrm{CH}_{3}\right), 22.7\left(\mathrm{CH}_{2}\right), 21.8\left(\mathrm{CH}_{2}\right), 18.00\left(\mathrm{CH}_{3}\right)$. IR (FTIR, neat) $v=3494,3073,2931$, $912 \mathrm{~cm}^{-1}$. HRMS (EI) Expected for $\mathrm{C}_{15} \mathrm{H}_{22}\left[\left(\mathrm{M}-\mathrm{H}_{2} \mathrm{O}\right)^{+}\right]: 202.1722$, Found: 202.1712

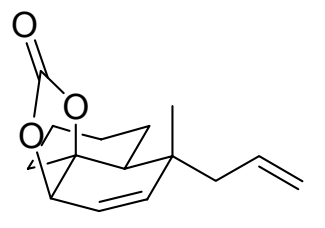

15

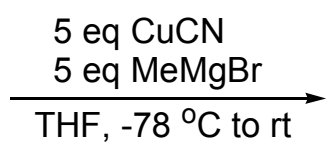

$68 \%$

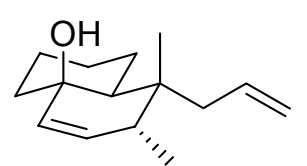

18

8-Allyl-7,8-dimethyl-1,3,4,7,8,8a-hexahydro-2H-naphthalen-4a-ol (18). A slurry of $\mathrm{CuCN}(1.06 \mathrm{~g}, 5 \mathrm{eq})$ in dry $\mathrm{THF}(120 \mathrm{~mL})$ under argon was cooled to $-78^{\circ} \mathrm{C}$, and $\mathrm{MeMgBr}(3 \mathrm{M}$ in $\mathrm{Et}_{2} \mathrm{O}, 3.97 \mathrm{~mL}, 5 \mathrm{eq}$ ) was added. The aspect of the mixture subtly changed from dull gray to 
bright white, as the mixture was allowed to stir for 45 minutes. The carbonate 15 (590 mg, 2.38 mmol) was added dropwise via syringe as a solution in dry THF $(10 \mathrm{~mL})$. No more dry ice was added to the cooling bath, and the reaction was allowed to warm gradually to room temperature over $\sim 4 \mathrm{~h}$. Stirring was continued overnight $(18 \mathrm{~h})$, and the reaction was quenched with $\mathrm{NH}_{4} \mathrm{Cl}_{\text {(sat.) }} / \mathrm{NH}_{4} \mathrm{OH}$ (9:1). Air was bubbled through the mixture for $1 \mathrm{~h}$ with vigorous stirring (to oxidize excess copper and aid with separation of the layers), and heterogeneous mixture was filtered. The organic layer was extracted with $\mathrm{Et}_{2} \mathrm{O}(4 \times 20 \mathrm{~mL})$, dried over $\mathrm{MgSO}_{4}$, and concentrated. The crude oil was subjected to flash chromatography (15\% EtOAc in Hexanes) to give 18 as a pale yellow oil ( $360 \mathrm{mg}, 68 \%$ ).

${ }^{1} \mathbf{H}$ NMR $\left(\mathrm{CDCl}_{3}, 500 \mathrm{MHz}\right) \delta_{\mathrm{ppm}}=5.88-5.78(\mathrm{~m}, 1 \mathrm{H}), 5.66(\mathrm{dd}, \mathrm{J}=4.2,14.1 \mathrm{~Hz}, 1 \mathrm{H}), 5.46(\mathrm{~d}, \mathrm{~J}$ $=9.9 \mathrm{~Hz}, 1 \mathrm{H}), 5.09-5.07(\mathrm{~m}, 1 \mathrm{H}), 5.07-5.04(\mathrm{~m}, 1 \mathrm{H}), 2.06(\mathrm{dd}, \mathrm{J}=8.0,14.1 \mathrm{~Hz}, 1 \mathrm{H}) 1.98-1.86$ $(\mathrm{m}, 3 \mathrm{H}), 1.86-1.81 \mathrm{~m}, 1 \mathrm{H}) 1.75-1.20(\mathrm{~m}, 7 \mathrm{H}), 0.96(\mathrm{~d}, \mathrm{~J}=0.75 \mathrm{~Hz}, 3 \mathrm{H}), 0.92(\mathrm{~d}, \mathrm{~J}=7.0 \mathrm{~Hz}, 3 \mathrm{H})$ ${ }^{13} \mathbf{C}$ NMR $\left(\mathrm{CDCl}_{3}, 125 \mathrm{MHz}\right) \delta_{\mathrm{ppm}}=135.2(\mathrm{CH}), 134.2(\mathrm{CH}), 131.4(\mathrm{CH}), 117.9(\mathrm{CH} 2), 69.4$ (C4), $45.3(\mathrm{CH}), 41.8(\mathrm{CH} 2), 40.2(\mathrm{CH} 2), 39.5(\mathrm{CH} 2), 36.1(\mathrm{C} 4), 26.9(\mathrm{CH} 2), 21.1(\mathrm{CH} 2), 20.7$ (CH) 19.9 (CH3), 15.5 (CH3). IR (FTIR, neat) v = 3485 (br), 3073 (m), 3008 (m), 2932 (s), 2874 (s), 1456 (s), 1442 (s), 1373 (s), 955 (m), 941 (s) cm $\mathrm{cm}^{-1}$. HRMS (EI) Calc for $\mathrm{C}_{15} \mathrm{H}_{24} \mathrm{O}_{1}\left[(\mathrm{M})^{+}\right]$: 220.18272, Found: 220.18232 (2.74\%).

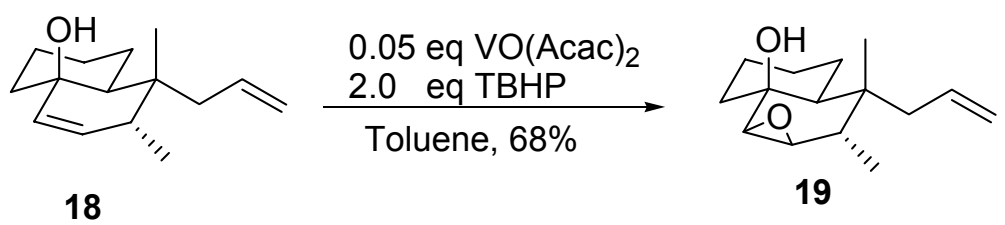

3-Allyl-2,3-dimethyl-octahydro-1-oxa-cyclopropa[a]naphthalen-7a-ol (19). A solution of the allylic alcohol 18 (39 mg, $177 \mathrm{umol})$ in toluene $(17 \mathrm{~mL})$ was stirred at room temperature and $\mathrm{VO}(\mathrm{Acac})_{2}(2.2 \mathrm{mg}, 0.05 \mathrm{eq})$ was added. After the catalyst had dissolved, TBHP (5-6M in pentane, $60 \mathrm{uL}, 2.0 \mathrm{eq}$ ) was added and the green solution became dark purple. The mixture was heated to $60{ }^{\circ} \mathrm{C}$ for $1 \mathrm{~h}$, and allowed to cool. A mixture of aqueous brine and $\mathrm{Na}_{2} \mathrm{SO}_{3}$ (sat'd, 1:1) were added, and stirred briefly prior to extraction with EtOAc ( 3 x $5 \mathrm{~mL}$ ), drying over $\mathrm{MgSO}_{4}$ and concentrating in vacuo. The residue was purified by flash chromatography (10\% EtOAc / Hexanes) to give $\mathbf{1 9}$ as a pale yellow oil (28 $\mathrm{mg}, 68 \%)$. 
${ }^{1} \mathbf{H}$ NMR $\left(\mathrm{CDCl}_{3}, 500 \mathrm{MHz}\right) \delta_{\mathrm{ppm}}=5.84-5.74(\mathrm{~m}, 1 \mathrm{H}), 5.12-5.03(\mathrm{~m}, 2 \mathrm{H}), 3.18(\mathrm{dd}, \mathrm{J}=2.1,3.7$ $\mathrm{Hz}, 1 \mathrm{H}), 2.96$ (d, J = 3.8 Hz), 2.07-2.03 (m, 2H), 1.95 (dd, J = 7.6, $14.1 \mathrm{~Hz}), 1.85-1.72(\mathrm{~m}, 4 \mathrm{H})$, 1.59-1.15 (m, 8H), $1.07(\mathrm{dd}, \mathrm{J}=2.7,12.3 \mathrm{~Hz}, 1 \mathrm{H}), 1.01(\mathrm{~d}, \mathrm{~J}=4.5 \mathrm{~Hz}, 3 \mathrm{H}), 1.01(\mathrm{~s}, 3 \mathrm{H}) .{ }^{13} \mathrm{C}$ NMR $\left(\mathrm{CDCl}_{3}, 125 \mathrm{MHz}\right) \delta_{\mathrm{ppm}}=134.7(\mathrm{CH}), 118.2\left(\mathrm{CH}_{2}\right), 68.8(\mathrm{C}$ QUAT. $), 61.4(\mathrm{CH}), 58.7$ $(\mathrm{CH}), 45.4(\mathrm{CH}), 43.8\left(\mathrm{CH}_{2}\right), 39.5\left(\mathrm{CH}_{2}\right), 35.9$ (C QUAT.), $35.2\left(\mathrm{CH}_{2}\right), 26.9\left(\mathrm{CH}_{2}\right), 21.4\left(\mathrm{CH}_{2}\right)$, $21.2\left(\mathrm{CH}_{3}\right), 20.0(\mathrm{CH}) 12.93\left(\mathrm{CH}_{3}\right)$. IR (FTIR, neat) v = 3500 (br), 3074, 2933, 1724, 1637, 1445, 1380, $973 \mathrm{~cm}^{-1}$. HRMS (EI) Calc for $\mathrm{C}_{15} \mathrm{H}_{24} \mathrm{O}_{2}\left[(\mathrm{M})^{+}\right]$: 236.17763, Found: 236.17663 $(2.74 \%)$.

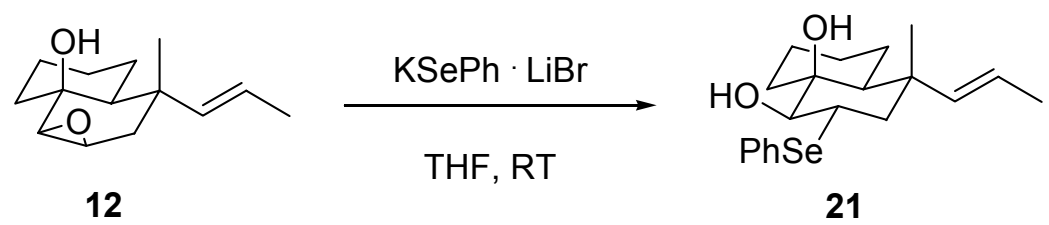

4-Methyl-2-phenylselanyl-4-propenyl-octahydro-naphthalene-1,8a-diol (21). A dry $250 \mathrm{~mL}$ roundbottom flask was fitted with a magnetic stirring bar and charged with $\mathrm{KBr}(5 \mathrm{eq})$. The flask was flame dried under high vacuum and the atmosphere replaced with argon. After cooling, diphenyl diselenide (1.28 g, $4.02 \mathrm{mmol}, 0.6 \mathrm{eq})$, and THF (70 mL) were added to give a canary yellow solution. $\mathrm{LiBH}_{4}(2 \mathrm{M}$ in THF, $4.70 \mathrm{~mL}, 1.4 \mathrm{eq})$ was added, with slow evolution of $\mathrm{H}_{2}$ noted. After the solution became colorless or white $(1 \mathrm{~h})$, a solution of the epoxide 12 (1.42 $\mathrm{g}$, $6.72 \mathrm{mmol}$ ) was added. After 2 hours, the reaction was deemed complete by TLC (20\% $\mathrm{Et}_{2} \mathrm{O} / \mathrm{Hexanes}$ as eluent). The reaction was quenched by slow addition of $\mathrm{NH}_{4} \mathrm{Cl}_{(\mathrm{aq})}$ and the organic layers were extracted with ether $(3 \times 20 \mathrm{~mL})$. Drying over $\mathrm{MgSO}_{4}$ and removal of the volatiles afforded a thick yellow sludge that was subjected to flash chromatography ( 0 to $20 \%$ $\mathrm{Et}_{2} \mathrm{O}$ in Hexanes) to give the selenide 21 as a thick, clear colourless oil (2.10 g, 86\%).

${ }^{1} \mathbf{H}$ NMR $\left(\mathrm{CDCl}_{3}, 500 \mathrm{MHz}\right) \delta_{\mathrm{ppm}}=7.58-7.55(\mathrm{~m}, 2 \mathrm{H}), 7.35-7.25(\mathrm{~m}, 3 \mathrm{H}), 7.32(\mathrm{dq}, \mathrm{J}=15.5,6.4$ Hz, 1H) $5.06(\mathrm{dd}, \mathrm{J}=15.5,1.4 \mathrm{~Hz}, 1 \mathrm{H}), 3.50(\mathrm{ddd}, \mathrm{J}=13.4,10.8,3.4 \mathrm{~Hz}, 1 \mathrm{H}), 2.98$ (d, J = 10.8 $\mathrm{Hz}, 1 \mathrm{H}) 1.97-1.92(\mathrm{~m}, 2 \mathrm{H}), 1.85(\mathrm{dd}, \mathrm{J}=13.4,3.4,1 \mathrm{H}) \mathrm{Hz}, 1.70-1.61(\mathrm{~m}, 1 \mathrm{H}) 1.61$ (dd, J = 6.4, $1.4 \mathrm{~Hz}, 3 \mathrm{H}), 1.57-1.24(\mathrm{~m}, 6 \mathrm{H}), 1.11(\mathrm{~s}, 3 \mathrm{H}), 1.11-0.85(\mathrm{~m}, 4 \mathrm{H}) .{ }^{13} \mathbf{C ~ N M R}\left(\mathrm{CDCl}_{3}, 125 \mathrm{MHz}\right)$ $\delta_{\mathrm{ppm}}=141.92(\mathrm{CH}), 136.28(\mathrm{CH}), 129.08(\mathrm{CH}), 128.34(\mathrm{CH}), 125.47$ (C QUAT.), $122.00(\mathrm{CH})$, $77.25(\mathrm{CH}), 73.52$ (C QUAT.), $49.21(\mathrm{CH}), 46.39\left(\mathrm{CH}_{2}\right), 45.77(\mathrm{CH}), 40.88(\mathrm{CH}), 37.81\left(\mathrm{CH}_{2}\right)$, 
$26.43\left(\mathrm{CH}_{2}\right), 22.09\left(\mathrm{CH}_{2}\right), 21.42\left(\mathrm{CH}_{2}\right), 18.33\left(\mathrm{CH}_{3}\right), 18.13\left(\mathrm{CH}_{3}\right)$. IR (FTIR, Neat) $v=3503$ (br), 3061, 3026, 2933, 2851, $978 \mathrm{~cm}^{-1}$. HRMS (EI) Expected for $\mathrm{C}_{20} \mathrm{H}_{28} \mathrm{O}_{2} \mathrm{Se}_{1}\left[(\mathrm{M})^{+}\right]: 380.1254$, found: $380.1272(40.1 \%)$
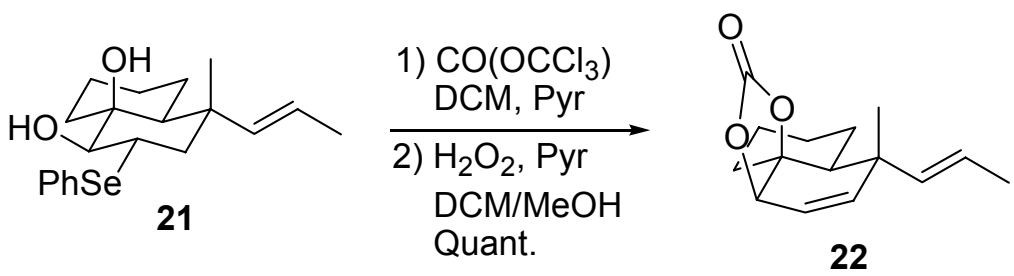

6-Allyl-6-methyl-6,6a,7,8,9,10-hexahydro-3aH-naphtho[1,8a-d][1,3]dioxol-2-one (22).

To a solution of triphosgene $(1.26 \mathrm{~g}, 1 \mathrm{eq})$ in dichloromethane $(50 \mathrm{~mL})$ under argon was added Pyridine $(3.45 \mathrm{~mL}, 10 \mathrm{eq})$ and cooled to $0{ }^{\circ} \mathrm{C}$. After stirring for several minutes, a white-yellow precipitate formed, and diol 21 (1.68 g, $4.42 \mathrm{mmol})$ was added via canula and rinse $(15 \mathrm{~mL}$ $\mathrm{CH}_{2} \mathrm{Cl}_{2}$ ). The reaction was stirred for $3 \mathrm{~h}$ while being allowed to come to room temperature. The reaction mixture was re-cooled to $0{ }^{\circ} \mathrm{C}$ and Methanol $(65 \mathrm{~mL})$ was added, followed by $\mathrm{H}_{2} \mathrm{O}_{2}$ ( $30 \%$ in $\mathrm{H}_{2} \mathrm{O}, 877 \mathrm{uL}, 2 \mathrm{eq}$ ). This was stirred for $1 \mathrm{~h}$ until the starting material had disappeared by TLC ( $\mathrm{rF}$ of the starting material is 0.6 in $20 \% \mathrm{EtOAc} / \mathrm{Hex}$, the oxidized selenide appears at the baseline). The oxidation was halted by the addition of water $(100 \mathrm{~mL})$, and the organic phase was extracted with dichloromethane $(4 \times 20 \mathrm{~mL})$. The combined organic phases were dried over $\mathrm{MgSO}_{4}$ and concentrated to leave a dark orange oily residue. The residue was redissolved in benzene $(60 \mathrm{~mL})$ and pyridine was added $(3.45 \mathrm{~mL}, 10 \mathrm{eq})$. The mixture was heated to $50-60{ }^{\circ} \mathrm{C}$ for one hour, the volatiles were removed, and the crude was subjected to flash chromatography (0 to $15 \% \mathrm{EtOAc} / \mathrm{Hex})$ to give $\mathbf{2 2}$ as light orange-red oil (1.10 g, quant).

${ }^{1}$ H NMR $\left(500 \mathrm{MHz}, \mathrm{CDCl}_{3}\right) 5.82(\mathrm{~d}, \mathrm{~J}=10.3 \mathrm{~Hz}, 1 \mathrm{H}), 5.57(\mathrm{dd}, \mathrm{J}=10.3,3.1 \mathrm{~Hz}, 1 \mathrm{H}), 5.42(\mathrm{dq}$, $\mathrm{J}=15.5,6.4 \mathrm{~Hz}), 5.26(\mathrm{dd}, \mathrm{J}=15.5,1.6 \mathrm{~Hz}), 4.59(\mathrm{~d}, \mathrm{~J}=3.1 \mathrm{~Hz}, 1 \mathrm{H}), 2.10-2.05(\mathrm{~m}, 1 \mathrm{H}), 1.89-$ $1.80(\mathrm{~m}, 1 \mathrm{H}), 1.77-1.66(\mathrm{~m}, 2 \mathrm{H}), 1.66(\mathrm{dd}, \mathrm{J}=6.4,1.6 \mathrm{~Hz}) 1.60-1.35(\mathrm{~m}, 4 \mathrm{H}), 1.30-1.25(\mathrm{~m}$, 1H), 1.07 (s, 3H); ${ }^{13} \mathbf{C}$ NMR (125 MHz, $\left.\mathrm{CDCl}_{3}\right) 154.1$ (O-(CO)-O), $143.1(\mathrm{CH}), 138.5(\mathrm{CH})$, $124.3(\mathrm{CH}), 118.3(\mathrm{CH}), 83.1$ (C QUAT.), $77.8(\mathrm{CH}), 45.6(\mathrm{CH}), 39.3$ (C QUAT.), $37.3\left(\mathrm{CH}_{2}\right)$, $25.6\left(\mathrm{CH}_{2}\right), 23.0\left(\mathrm{CH}_{2}\right), 21.4\left(\mathrm{CH}_{2}\right), 21.0\left(\mathrm{CH}_{3}\right), 18.1\left(\mathrm{CH}_{3}\right)$ IR (FTIR, Neat) $v=3027(\mathrm{~m}), 2939$ (s), $2865(\mathrm{~m}), 1801(\mathrm{~s}), 1449$ (m), 1205 (s), 1011 (s), 1027 (s) $\mathrm{cm}^{-1}$. HRMS (EI) Expected for $\mathrm{C}_{15} \mathrm{H}_{20} \mathrm{O}_{3}\left[(\mathrm{M})^{+}\right]$: 248.14125 , found: 248.14130 (40.1\%). 


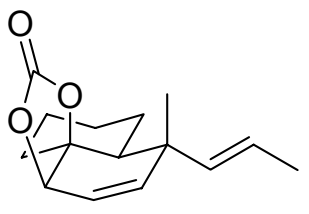

22
1) $\mathrm{CuCN}, \mathrm{MeMgBr}$ 2) $\mathrm{VO}(\mathrm{Acac})_{2}$, TBHP Toluene $61 \%$

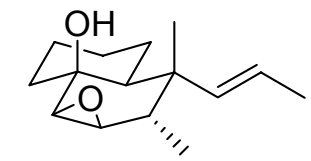

20

2,3-Dimethyl-3-propenyl-octahydro-1-oxa-cyclopropa[a]naphthalen-7a-ol (20). A dry $500 \mathrm{~mL}$ flask under argon was fitted with a magnetic sturring bar, and charged with $\mathrm{CuCN}$ (1.50 g, 5 eq). Dry THF $(0.1 \mathrm{M}$ in $\mathrm{Cu}, 140 \mathrm{~mL})$ was added and the slurry was cooled to $-78^{\circ} \mathrm{C}$. $\mathrm{MeMgBr}(3 \mathrm{M}$ in Ether, $5.58 \mathrm{~mL})$ was added and stirring was continued for 45 minutes. A solution of the allylic carbonate $22(830 \mathrm{mg}, 3.35 \mathrm{mmol})$ in dry THF $(20 \mathrm{~mL})$ was added dropwise. No more dry ice was added to the cold bath, and the reaction was allowed to come to room temperature overnight $(18 \mathrm{~h})$. The cuprate was quenched with saturated $\mathrm{NH}_{4} \mathrm{Cl}_{(\mathrm{aq})}: \mathrm{NH}_{4} \mathrm{OH}$ $(8: 1)(30 \mathrm{~mL})$, and allowed to stir for 2 hours. The organic layers were extracted with ether $(3 \mathrm{x}$ $30 \mathrm{~mL})$, combined, and washed with brine $(2 \times 30 \mathrm{~mL})$. After drying over $\mathrm{MgSO}_{4}$ and removal of the volatiles, the remaining yellow oil was purified to give. The procedure for epoxidation used was identical above (see compound 11 or 19), but was completed after only 45 minutes.

Purification by flash chromatography (0 to 15\% Ether in Hexanes) afforded the epoxide 20 (482 $\mathrm{mg}, 61 \%$ ) as a colourless oil.

${ }^{1} \mathbf{H}$ NMR $\left(\mathrm{CDCl}_{3}, 500 \mathrm{MHz}\right) \delta_{\mathrm{ppm}}=5.27(\mathrm{dq}, \mathrm{J}=15.7,6.3 \mathrm{~Hz}, 1 \mathrm{H}), 5.14(\mathrm{dd}, \mathrm{J}=1.5,15.7 \mathrm{~Hz}$, 1H), $3.21(\mathrm{dd}, \mathrm{J}=1.7,3.9 \mathrm{~Hz}, 1 \mathrm{H}), 3.02(\mathrm{~d}, \mathrm{~J}=3.9 \mathrm{~Hz}, 1 \mathrm{H}), 2.10-2.02$ (m, 1H), 1.97 (dq, J = 1.7, $7.5 \mathrm{~Hz}, 1 \mathrm{H}) 1.85-1.73(\mathrm{~m}, 3 \mathrm{H}), 1.67$ (dd, J = 1.5, $6.3 \mathrm{~Hz}, 3 \mathrm{H}) 1.55(\mathrm{~s}, 3 \mathrm{H}), 1.50-1.40(\mathrm{~m}, 2 \mathrm{H})$, 1.30-1.04 (m, 4H), $0.96(\mathrm{~d}, \mathrm{~J}=7.5 \mathrm{~Hz}, 3 \mathrm{H}) .{ }^{13} \mathbf{C ~ N M R}\left(\mathrm{CDCl}_{3}, 125 \mathrm{MHz}\right) \delta_{\mathrm{ppm}}=140.5(\mathrm{CH})$, $122.1(\mathrm{CH}), 68.8\left(\mathrm{C}_{4}\right), 61.7(\mathrm{CH}), 59.0(\mathrm{CH}), 45.5(\mathrm{CH}), 39.5\left(\mathrm{CH}_{2}\right), 39.0(\mathrm{CH}), 38.7\left(\mathrm{C}_{4}\right), 27.0$ $\left(\mathrm{CH}_{2}\right), 21.7\left(\mathrm{CH}_{2}\right), 21.4\left(\mathrm{CH}_{2}\right), 20.8\left(\mathrm{CH}_{3}\right), 18.2\left(\mathrm{CH}_{3}\right), 14.4\left(\mathrm{CH}_{3}\right)$, IR (FTIR, Neat) $v=3593$

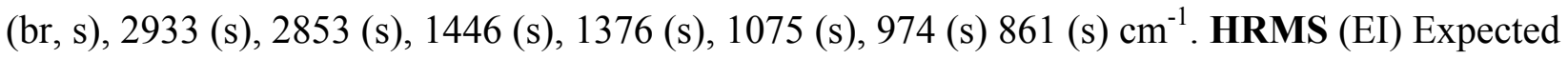
for $\mathrm{C}_{15} \mathrm{H}_{24} \mathrm{O}_{2}\left[(\mathrm{M})^{+}\right]$: 236.17763, Found: 236.17660 (2.2\%) 


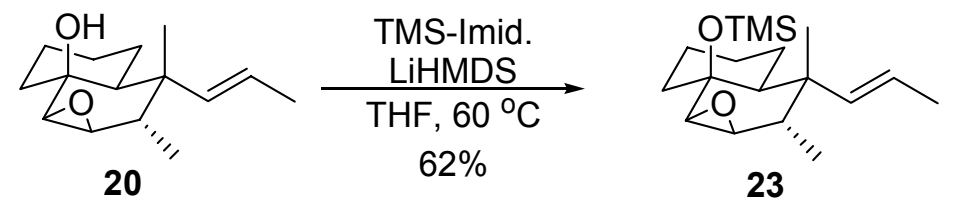

\section{2,3-Dimethyl-3-propenyl-octahydro-1-oxa-cyclopropa[a]naphthalen-7a-yloxy)-trimethyl-} silane (23). A solution of free alcohol $20(660 \mathrm{mg}, 2.80 \mathrm{mmol})$ in dry THF (30 mL) was stirred at room temperature under argon and TMS Imidazole ( $820 \mathrm{uL}, 2 \mathrm{eq})$ was added, followed by LiHMDS (450 mg, $1 \mathrm{eq}$ ) in one portion. The mixture was heated to reflux for $1 \mathrm{~h}$ and allowed to cool. Silica was added to the reaction mixture $(\sim 1 \mathrm{~g})$ and the volatiles were removed on rotovap and hi-vac. The residual powder was subjected to flash chromatography ( 0 to $5 \% \mathrm{Et}_{2} \mathrm{O}$ in Hexanes) to give 23 as a yellow oil (538 $\mathrm{mg}, 62 \%)$.

${ }^{1} \mathbf{H}$ NMR $\left(\mathrm{CDCl}_{3}, 500 \mathrm{MHz}\right) \delta_{\mathrm{ppm}}=5.25(\mathrm{dq}, \mathrm{J}=15.6,6.2 \mathrm{~Hz}, 1 \mathrm{H}), 5.16(\mathrm{dd}, \mathrm{J}=15.6,1.2 \mathrm{~Hz}$, 1H), 3.04 (dd, J = 3.8, 1.5 Hz, 1H), 2.87 (d, J=3.8 Hz), 1.92-1.65 (m, 4H), 1.65 (dd, J = 6.2, 1.2 $\mathrm{Hz}, 3 \mathrm{H}), 1.65-1.34(\mathrm{~m}, 4 \mathrm{H}), 1.33-1.12(\mathrm{~m}, 2 \mathrm{H}), 1.03(\mathrm{~s}, 3 \mathrm{H}), 0.93$ (d, J=10.0 Hz, 3H), $0.16(\mathrm{~s}$, 9H). ${ }^{13} \mathbf{C ~ N M R}\left(\mathrm{CDCl}_{3}, 125 \mathrm{MHz}\right) \delta_{\mathrm{ppm}}=141.32(\mathrm{CH}), 121.04(\mathrm{CH}), 72.276$ (C QUAT.), 60.47 $(\mathrm{CH}), 58.26(\mathrm{CH}), 46.89(\mathrm{CH}), 40.319(\mathrm{CH}), 39.56\left(\mathrm{CH}_{2}\right), 38.76(\mathrm{CH}), 27.12\left(\mathrm{CH}_{2}\right), 27.96$ $\left(\mathrm{CH}_{2}\right), 21.31\left(\mathrm{CH}_{2}\right), 20.20(\mathrm{CH}), 18.23\left(\mathrm{CH}_{3}\right), 14.52\left(\mathrm{CH}_{3}\right), 2.21\left(3 \mathrm{x} \mathrm{CH}_{3}\right)$. IR $(\mathrm{FTIR}, \mathrm{Neat}) \mathrm{v}=$ 3209 (w), 2939 (s), 1248 (s), 1034 (s), 839 (s) $\mathrm{cm}^{-1}$. HRMS (EI) Expected for $\mathrm{C}_{18} \mathrm{H}_{32} \mathrm{O}_{2} \mathrm{Si}\left[(\mathrm{M})^{+}\right]$: 308.2172, Found: 308.2170

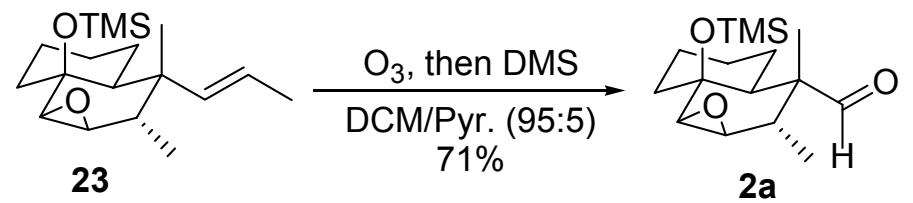

\section{2,3-Dimethyl-7a-trimethylsilanyloxy-decahydro-1-oxa-cyclopropa[a]naphthalene-3-} carbaldehyde (2a). A clean dry flask was charged with olefin 23 (538 mg, 1.72 mmol), dichloromethane $(17 \mathrm{~mL})$ and Pyridine $(850 \mathrm{uL})$. The solution was bubbled with oxygen while being cooled to $-78^{\circ} \mathrm{C}$. Once cool, the current was switched on and ozone was generated until the steel-gray ozone colour persisted (approx. 30 minutes). The solution was sparged with Argon for 20 minutes and DMS (5 eq) was added. The reaction was allowed to warm to room temperature, and brine $(5 \mathrm{~mL})$ was added. The organic phase was extracted with $\mathrm{CH}_{2} \mathrm{Cl}_{2}(3 \times 10 \mathrm{~mL})$, dried 
over $\mathrm{MgSO}_{4}$ and concentrated. The crude was subjected to flash chromatography (0 to $20 \%$ EtOAc in Hexanes) to give the aldehyde $2 \mathbf{a}$ as a thick colourless oil (360 $\mathrm{mg}, 71 \%)$.

${ }^{1} \mathbf{H}$ NMR $\left(\mathrm{CDCl}_{3}, 500 \mathrm{MHz}\right) \delta_{\mathrm{ppm}}=9.35(\mathrm{~s}, 1 \mathrm{H}), 3.03(\mathrm{dd}, \mathrm{J}=3.7,1.4 \mathrm{~Hz}, 1 \mathrm{H}), 2.94(\mathrm{~d}, \mathrm{~J}=3.7$ $\mathrm{Hz}, 1 \mathrm{H}), 2.19$ (dq, J = 7.5, 1.1 Hz, $1 \mathrm{H}), 1.90-1.65$ (m, 3H), 1.64-1.40 (m, 4H), 1.29-1.20 (m, 2H), $1.1(\mathrm{~s}, 3 \mathrm{H}), 1.00(\mathrm{~d}, \mathrm{~J}=7.5 \mathrm{~Hz}, 3 \mathrm{H}), 0.16(\mathrm{~s}, 9 \mathrm{H}) .{ }^{13} \mathbf{C} \mathbf{N M R}\left(\mathrm{CDCl}_{3}, 125 \mathrm{MHz}\right) \delta_{\mathrm{ppm}}=$ $207.8(\mathrm{CH}), 71.6$ (C QUAT.), $59.8(\mathrm{CH}), 58.1(\mathrm{CH}), 48.3$ (C QUAT.), $42.7(\mathrm{CH}), 40.0\left(\mathrm{CH}_{2}\right)$, $35.9(\mathrm{CH}), 26.5\left(\mathrm{CH}_{2}\right), 21.8\left(\mathrm{CH}_{2}\right), 21.7\left(\mathrm{CH}_{2}\right), 16.6\left(\mathrm{CH}_{3}\right), 13.6\left(\mathrm{CH}_{3}\right), 2.1\left(3 \mathrm{x} \mathrm{CH}_{3}\right)$. IR (FTIR, Neat) $v=2945$ (s), 2859 (m), 1727 (s), 1445 (m), 1248 (s), 1031 (s), 839 (s) cm cm $^{-1}$ HRMS (EI) Expected for $\mathrm{C}_{16} \mathrm{H}_{28} \mathrm{O}_{3} \mathrm{Si}\left[(\mathrm{M})^{+}\right]$: 296.18077, found: 296.17800 .

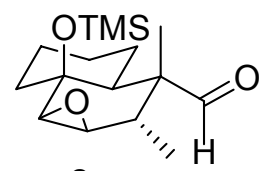

$2 a$

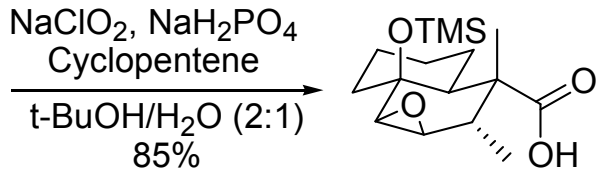

24

2,3-Dimethyl-7a-trimethylsilanyloxy-decahydro-1-oxa-cyclopropa[a]naphthalene-3carboxylic acid (24). A solution of $\mathbf{2 a}(76 \mathrm{mg}, 257 \mathrm{umol})$ in $\mathrm{t}-\mathrm{BuOH}(2 \mathrm{~mL})$ and $\mathrm{H}_{2} \mathrm{O}(1 \mathrm{~mL})$ was stirred at $0{ }^{\circ} \mathrm{C}$ and $\mathrm{NaH}_{2} \mathrm{PO}_{4}(308 \mathrm{mg}, 10 \mathrm{eq})$ was added. Once the solid had dissolved, cyclopentene $(100 \mathrm{uL})$ was added, followed by $\mathrm{NaClO}_{2}(140 \mathrm{mg}, 6 \mathrm{eq})$. The reaction was stirred vigorously. After 30 minutes, the reaction was allowed to warm to ambient temperature, and stirred for another $30 \mathrm{~min}$. The reaction mixture was returned to an ice bath, and a mixture of $\mathrm{Na}_{2} \mathrm{SO}_{3 \text { (sat.) }}$ and Brine $(1: 1,5 \mathrm{~mL})$ was added. The organic phase was extracted with EtOAc $(3 \times 5$ $\mathrm{mL}$ ), dried over $\mathrm{MgSO}_{4}$ and concentrated. The crude was passed through a pad of silica (40\% EtOAc in Hexanes) to give 24 as a white powder. (63 $\mathrm{mg}, 85 \%)$.

${ }^{1} \mathbf{H}$ NMR $\left(\mathrm{CDCl}_{3}, 500 \mathrm{MHz}\right) \delta_{\mathrm{ppm}}=3.05(\mathrm{dd}, \mathrm{J}=2.1,3.9 \mathrm{~Hz}, 1 \mathrm{H}), 2.92(\mathrm{~d}, \mathrm{~J}=3.9 \mathrm{~Hz}, 1 \mathrm{H}), 2.14$ $(\mathrm{dq}, \mathrm{J}=2.1,7.5 \mathrm{~Hz}, 1 \mathrm{H}), 1.90-1.55(\mathrm{~m}, 6 \mathrm{H}), 1.50-1.35(\mathrm{~m}, 4 \mathrm{H}), 1.25$ (s, 3H), 0.98 (d, J = 7.4 Hz, $3 \mathrm{H}), 0.15(\mathrm{~s}, 9 \mathrm{H}) .{ }^{13} \mathbf{C} \mathbf{N M R}\left(\mathrm{CDCl}_{3}, 500 \mathrm{MHz}\right) \delta_{\mathrm{ppm}}=182.3,71.9,58.9,58.0,48.2,45.7,40.3$, 37.2, 26.6, 23.7, 21.8, 19.0, 15.2, 2.6. IR (FTIR, Neat) v = 3429 (br), 2946 (s), 2858 (m), 1698 (s), 1445 (m), 1248 (s), 1049 (s), 840 (s) cm cm $^{-1}$ HRMS (EI) Expected for $\mathrm{C}_{16} \mathrm{H}_{28} \mathrm{O}_{4} \mathrm{Si}\left[(\mathrm{M})^{+}\right]$: 312.17569 , found: 312.17211 (1.4\%). 

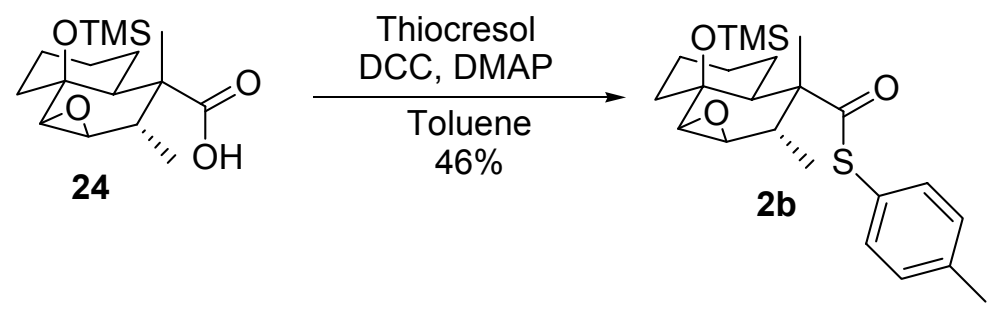

\section{2,3-Dimethyl-7a-trimethylsilanyloxy-decahydro-1-oxa-cyclopropa[a]naphthalene-3-}

carbothioic acid S-p-tolyl ester (2b). A solution of acid $24(63 \mathrm{mg})$ in Toluene $(5 \mathrm{~mL})$ was stirred at room temperature and dicyclohexyl carbodiimide $\left(1 \mathrm{M}\right.$ in $\mathrm{CH}_{2} \mathrm{Cl}_{2}, 5$ eq) was added, dropwise, followed by thiocresol (50 mg, $2 \mathrm{eq})$ and DMAP $(1 \mathrm{mg})$. The reaction was stirred at ambient temperature for $18 \mathrm{~h}$, and diluted with ether $(10 \mathrm{~mL})$. The mixture was filtered through a sintered glass filter. The organic phase was washed with water $(2 \times 2 \mathrm{~mL})$ and brine $(1 \times 2 \mathrm{~mL})$. The layer was dried over $\mathrm{MgSO}_{4}$ and concentrated. The residue was subjected to flash chromatography (0 to $10 \% \mathrm{Et}_{2} \mathrm{O}$ in Hexanes) to afford $\mathbf{2 b}$ as a thick yellow oil. (38 $\mathrm{mg}, 46 \%$ ) ${ }^{1} \mathbf{H}$ NMR $\left(\mathrm{CDCl}_{3}, 500 \mathrm{MHz}\right) \delta_{\mathrm{ppm}}=7.26-1.15(\mathrm{~m}, 5 \mathrm{H}), 3.10(\mathrm{dd}, \mathrm{J}=1.9,3.8 \mathrm{~Hz}, 1 \mathrm{H}), 2.89(\mathrm{~d}, \mathrm{~J}=$ $3.8 \mathrm{~Hz}, 1 \mathrm{H}), 2.34$ (s, 3H), $2.26(\mathrm{dq}, \mathrm{J}=1.8,7.4 \mathrm{~Hz}, 1 \mathrm{H}), 1.92-1.55(\mathrm{~m}, 9 \mathrm{H}), 1.46(\mathrm{~s}, 3 \mathrm{H}), 0.95$ (d, $\mathrm{J}=7.4 \mathrm{~Hz}, 3 \mathrm{H}), 0.17$ (s, $9 \mathrm{H}) .{ }^{13} \mathbf{C} \mathbf{N M R}\left(\mathrm{CDCl}_{3}, 125 \mathrm{MHz}\right) \delta_{\mathrm{ppm}}=204.1$ (C QUAT.), 139.4 (C QUAT.), 134.9 (CH), 134.8 (CH), 130.0 (CH), 129.9 (CH), 124.8 (C QUAT.), 72.3 (C QUAT.), $59.6(\mathrm{CH}), 58.0\left(\mathrm{CH}_{3}\right), 55.7$ (C QUAT.), $45.0(\mathrm{CH}), 40.2\left(\mathrm{CH}_{2}\right), 37.7(\mathrm{CH}), 26.6\left(\mathrm{CH}_{2}\right), 22.5$ $\left(\mathrm{CH}_{2}\right), 21.8\left(\mathrm{CH}_{2}\right), 21.3(\mathrm{CH}), 19.5\left(\mathrm{CH}_{3}\right), 15.3\left(\mathrm{CH}_{3}\right), 2.2\left(3 \mathrm{x} \mathrm{CH}_{3}\right)$. IR (FTIR, Neat) v $=2943$ (s), 2857 (m), 1696 (s), 1445 (m), 1258 (s), 1247 (s), 1108 (s), 838 (s) cm². HRMS (EI) Expected for $\mathrm{C}_{16} \mathrm{H}_{27} \mathrm{O}_{3} \mathrm{Si}\left[(\mathrm{M}-\mathrm{STol})^{+}\right]$: 295.1730, found: 295.1737 (3.7\%). 


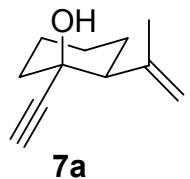

${ }^{1} \mathbf{H}$ NMR $\left(\mathrm{CDCl}_{3}, 300 \mathrm{MHz}\right)$

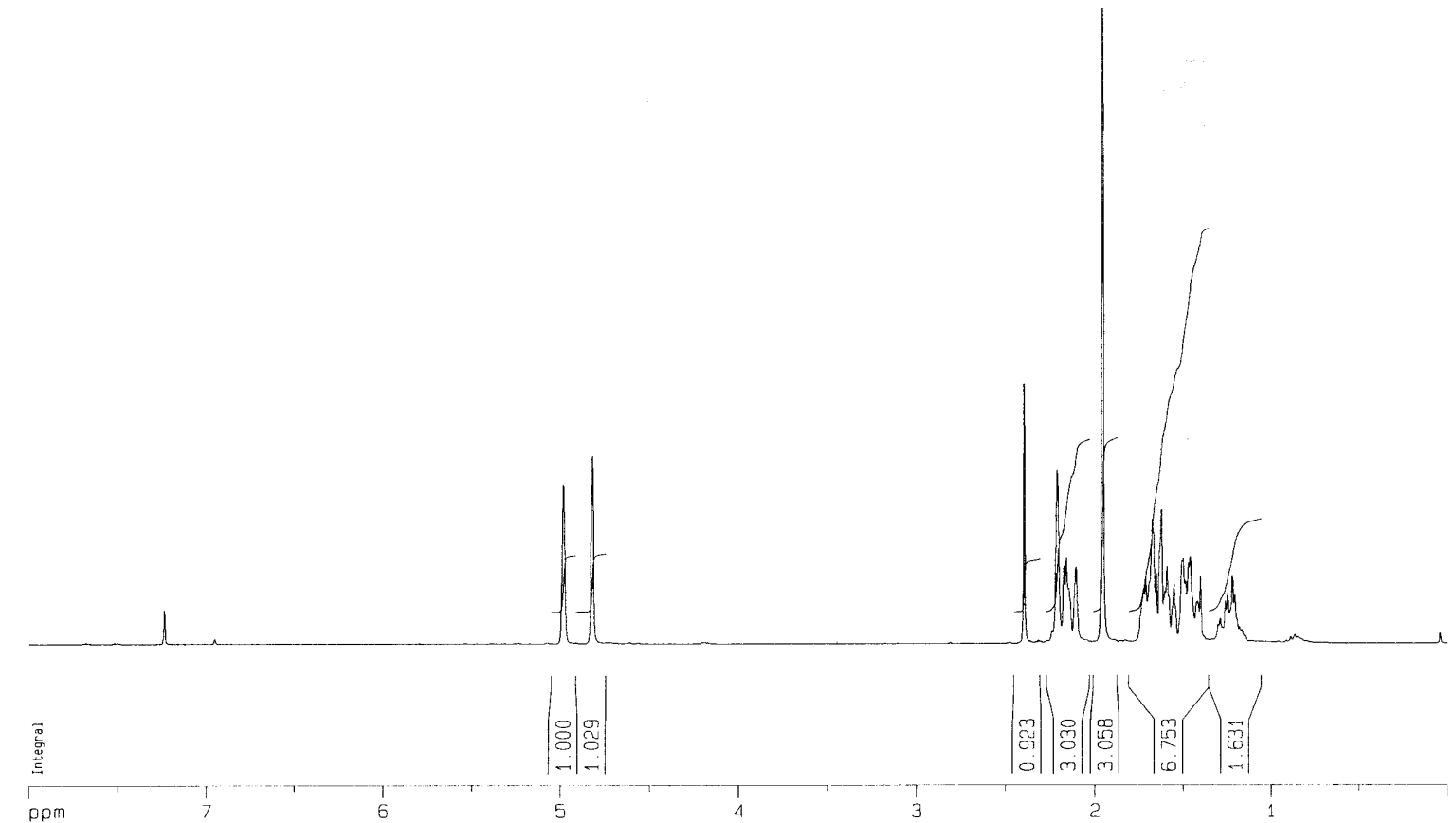

${ }^{13} \mathbf{C}$ NMR $\left(\mathrm{CDCl}_{3}, 75 \mathrm{MHz}\right)$

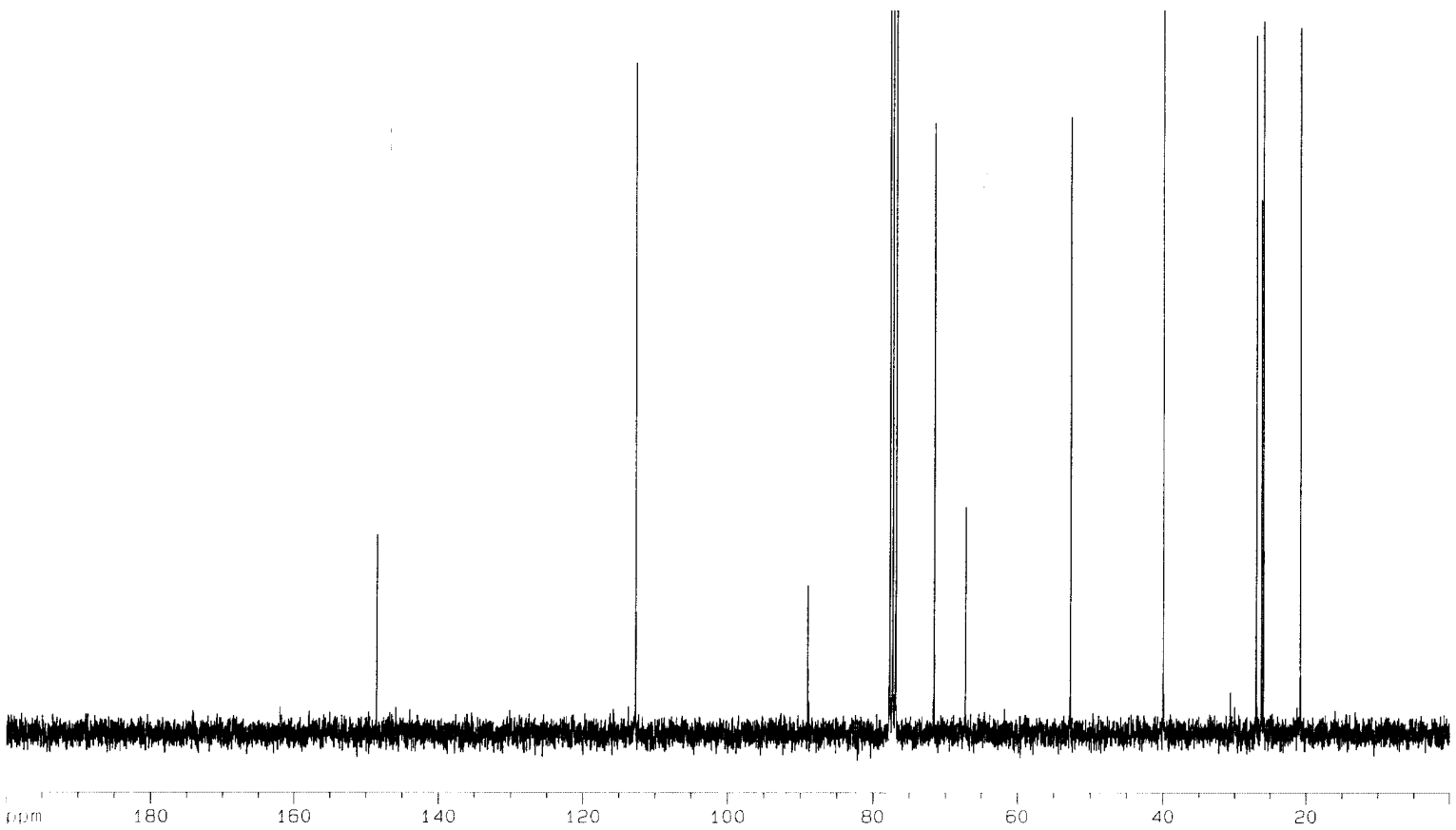




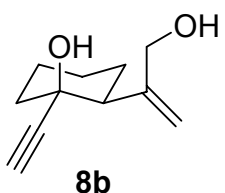

${ }^{1} \mathbf{H}$ NMR $\left(\mathrm{CDCl}_{3}, 300 \mathrm{MHz}\right)$

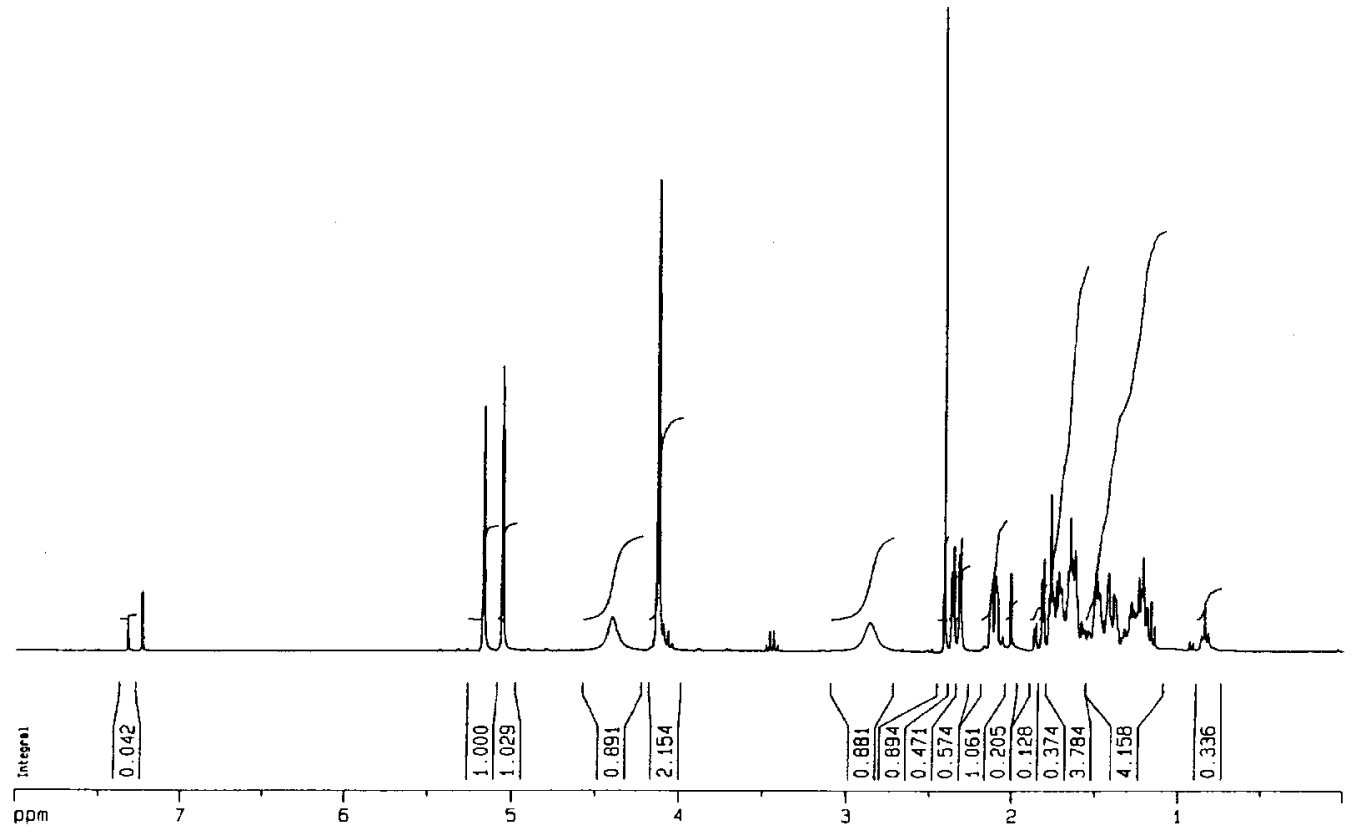

${ }^{13} \mathbf{C}$ NMR $\left(\mathrm{CDCl}_{3}, 75 \mathrm{MHz}\right)$

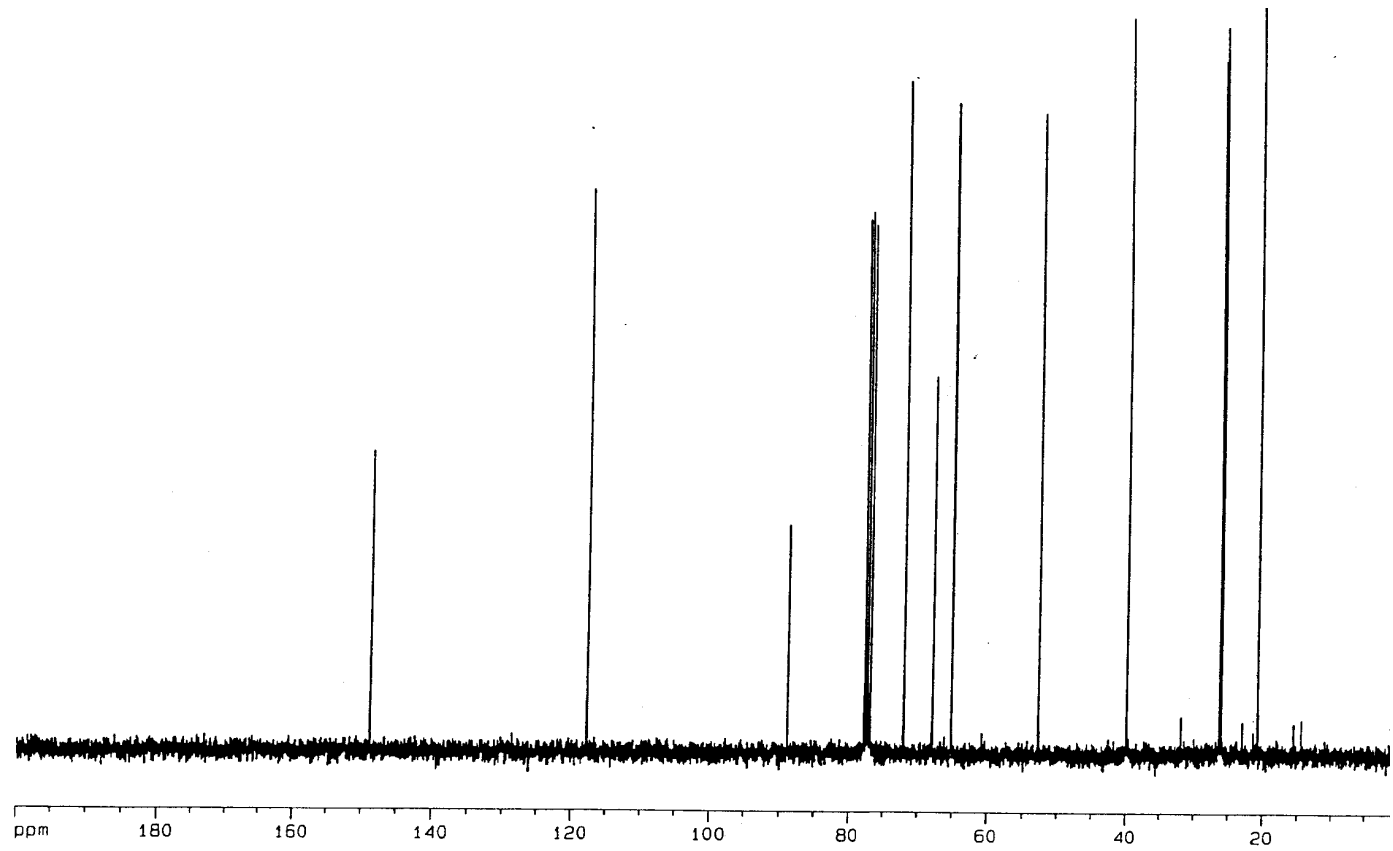




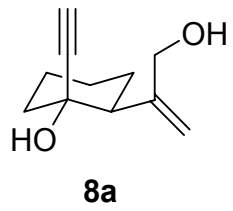

${ }^{1} \mathbf{H}$ NMR $\left(\mathrm{CDCl}_{3}, 300 \mathrm{MHz}\right)$

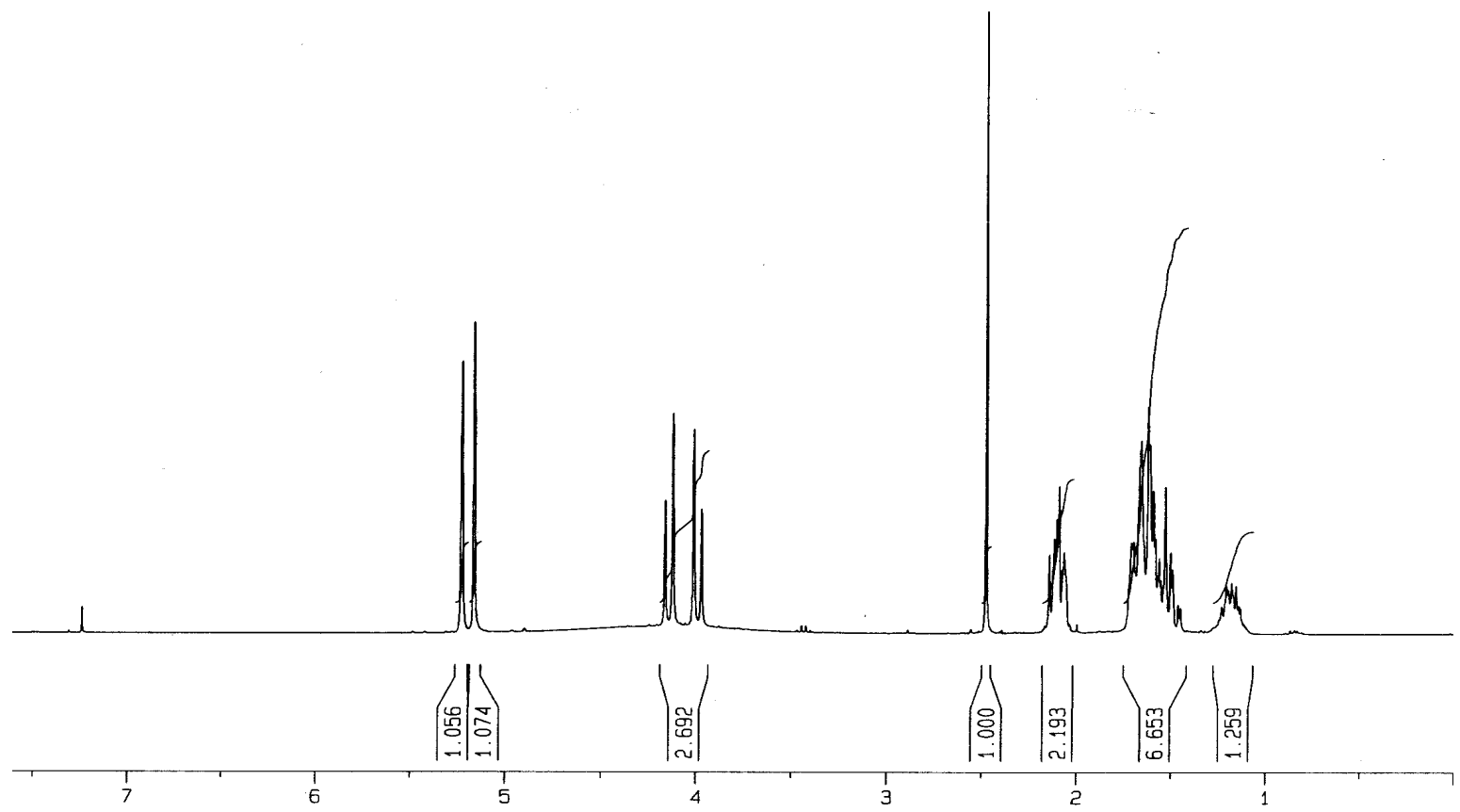

${ }^{13} \mathbf{C}$ NMR $\left(\mathrm{CDCl}_{3}, 75 \mathrm{MHz}\right)$

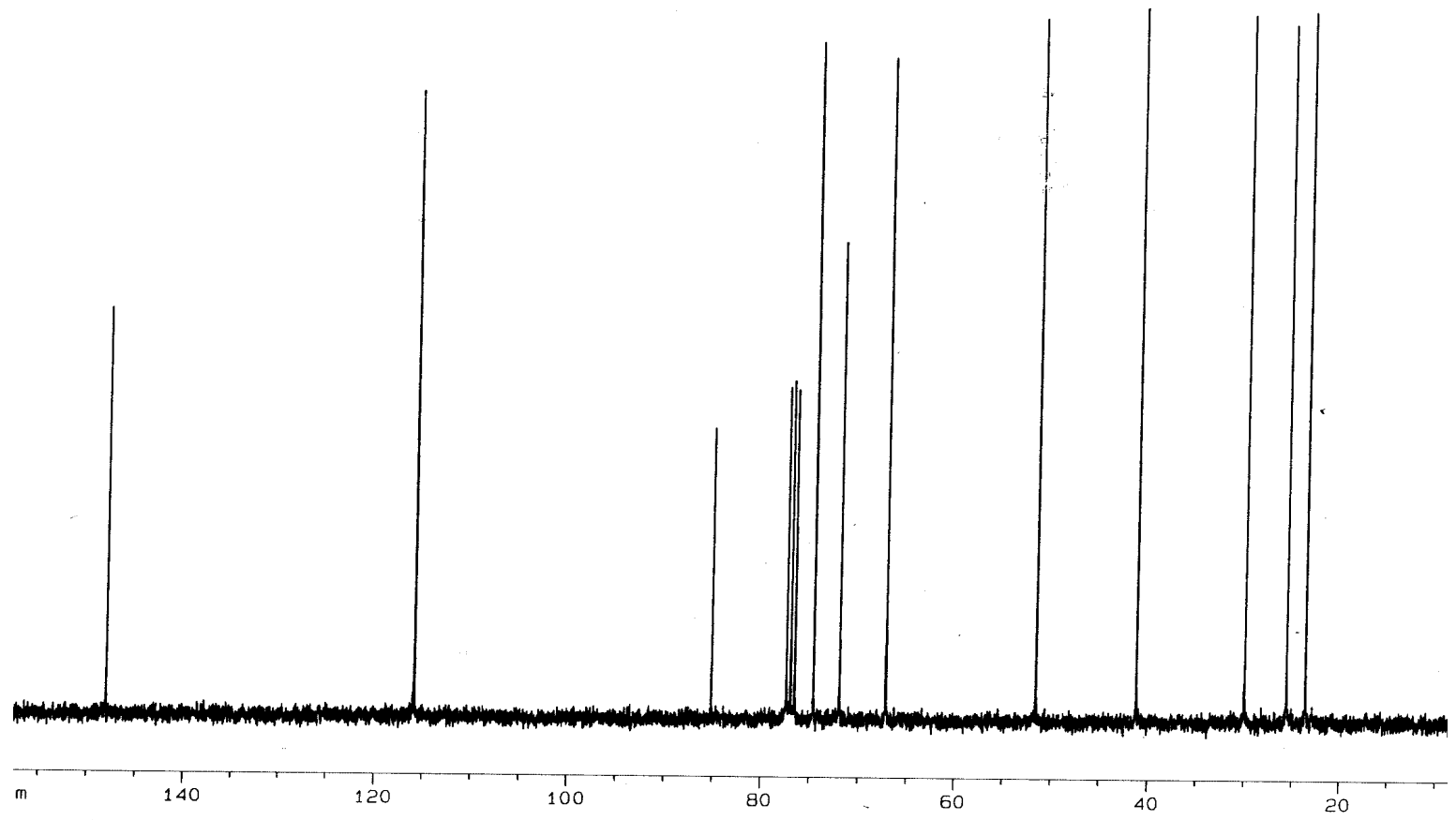




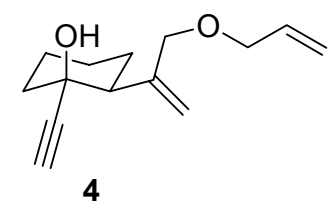

${ }^{1} \mathbf{H}$ NMR $\left(\mathrm{CDCl}_{3}, 300 \mathrm{MHz}\right)$

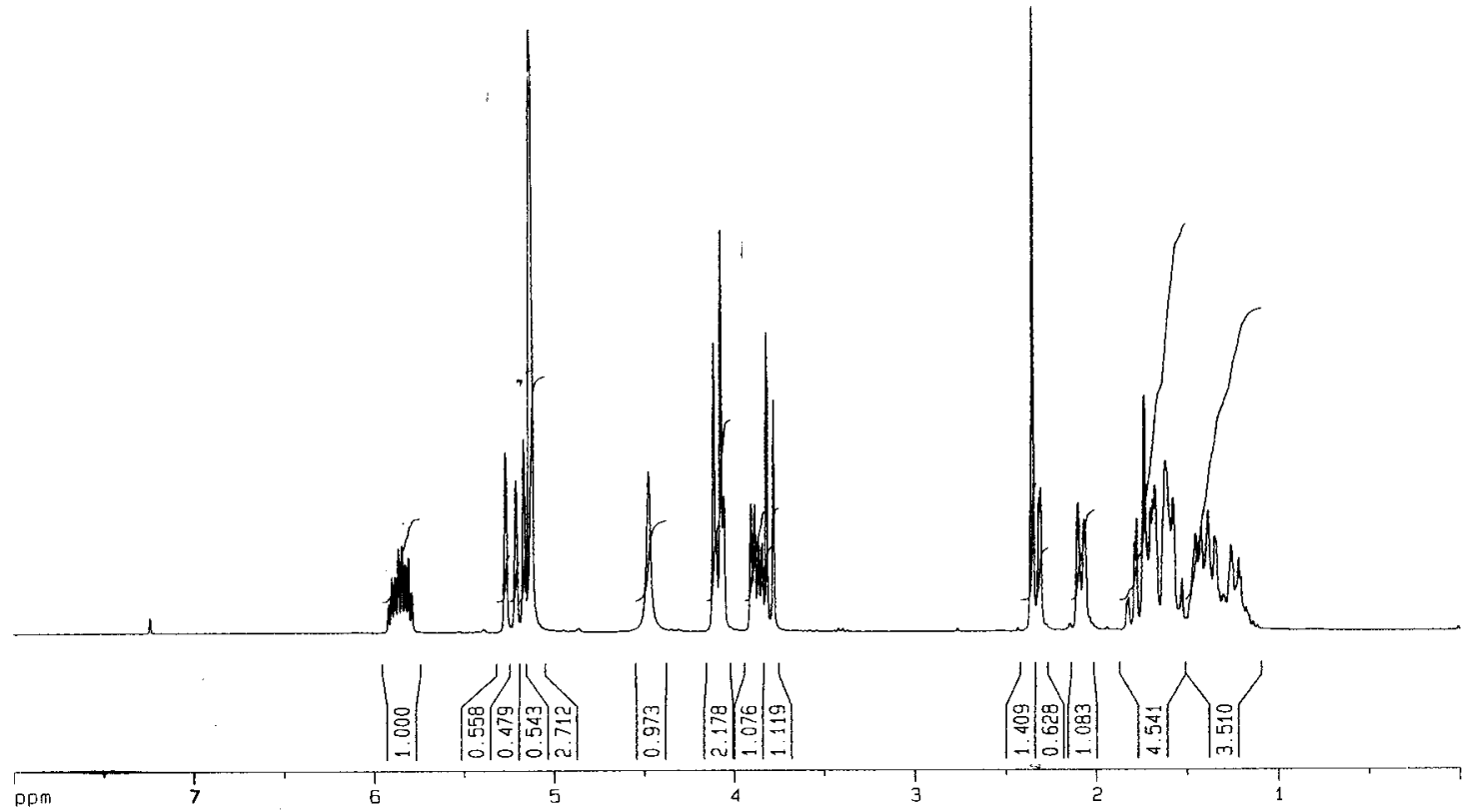

${ }^{13} \mathbf{C}$ NMR $\left(\mathrm{CDCl}_{3}, 75 \mathrm{MHz}\right)$

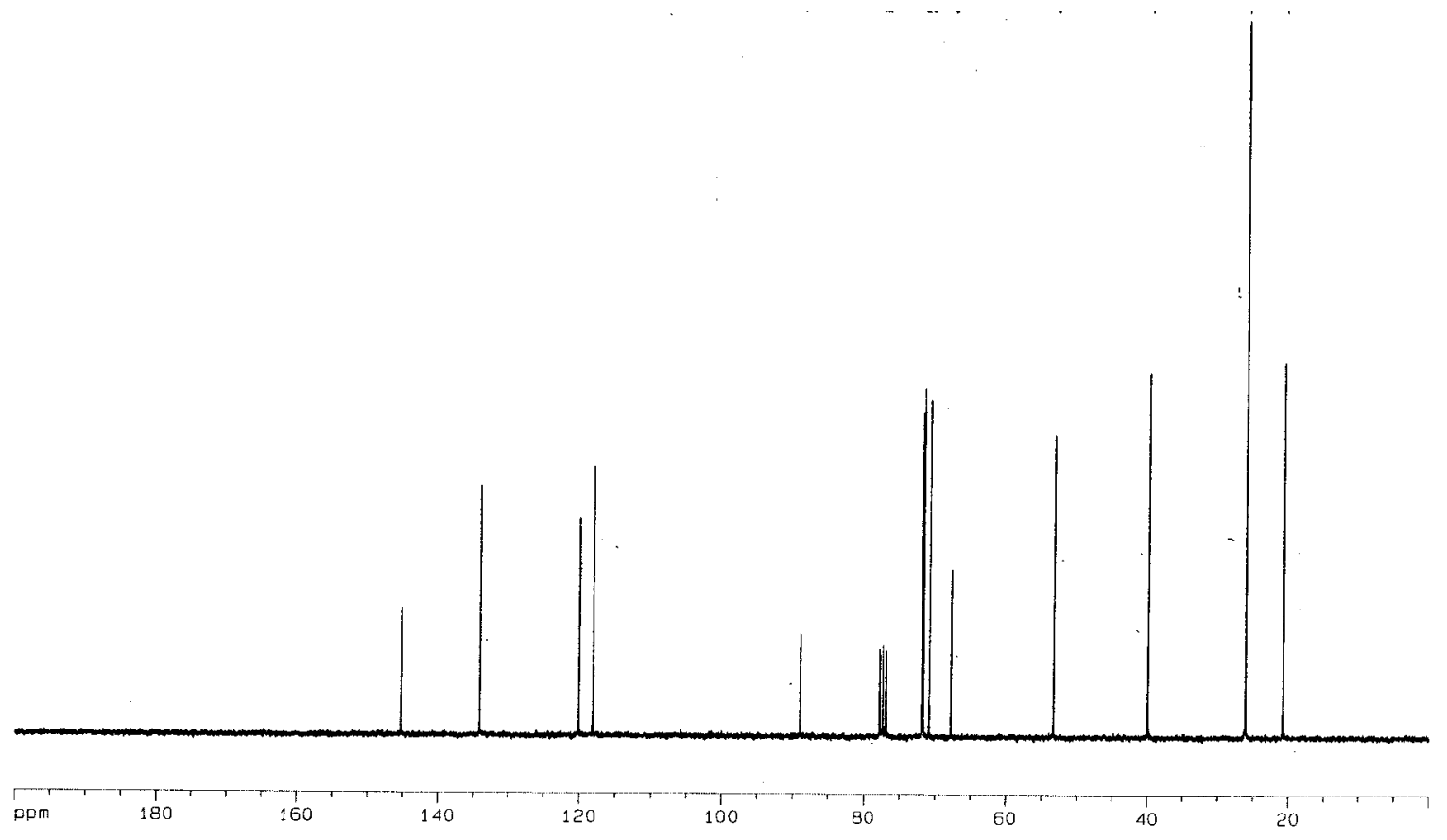




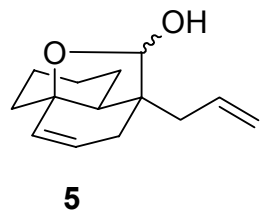

${ }^{1} \mathbf{H} \mathbf{N M R}\left(\mathrm{CDCl}_{3}, 300 \mathrm{MHz}\right)$

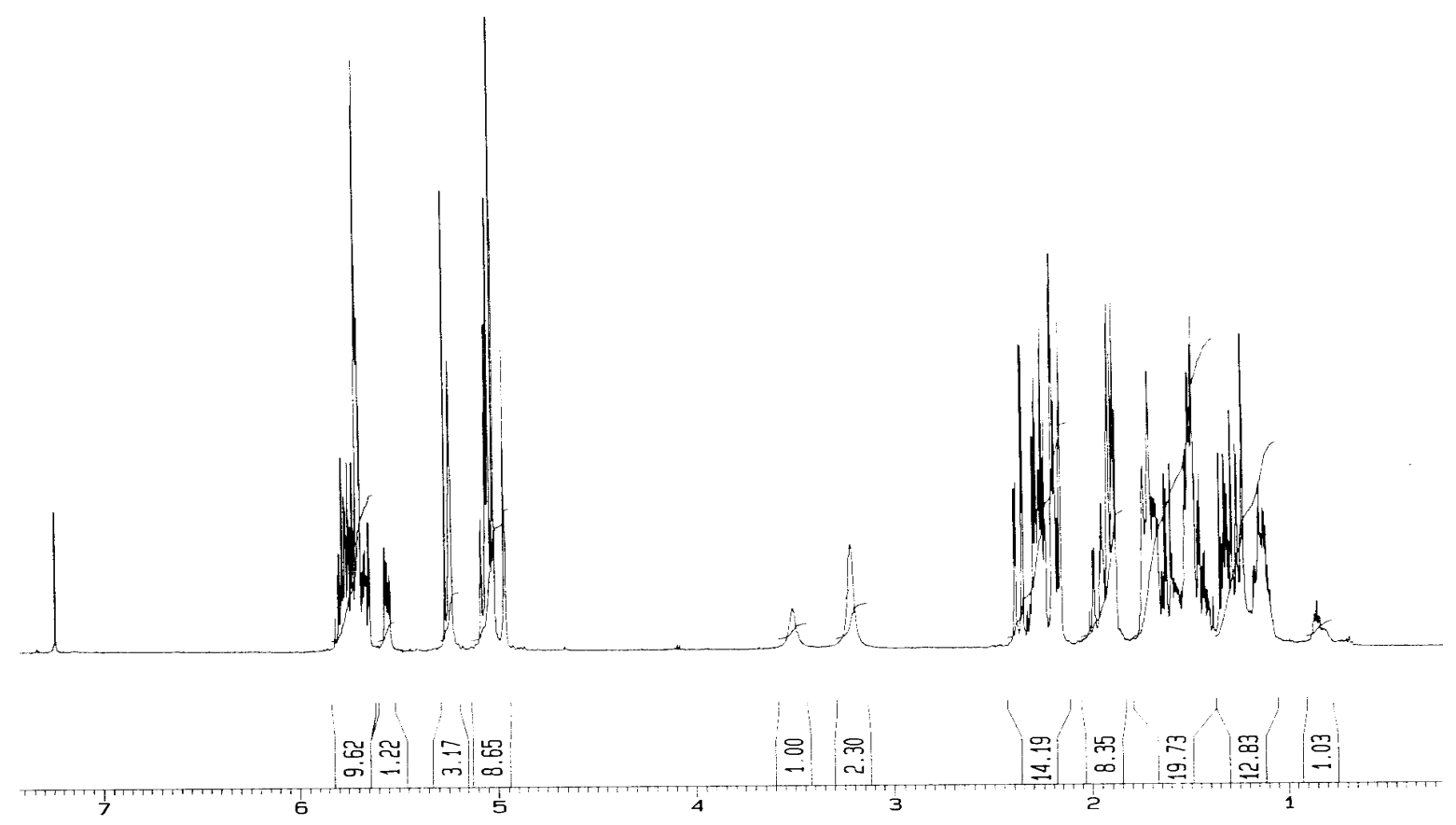

${ }^{13} \mathbf{C}$ NMR $\left(\mathrm{CDCl}_{3}, 75 \mathrm{MHz}\right)$

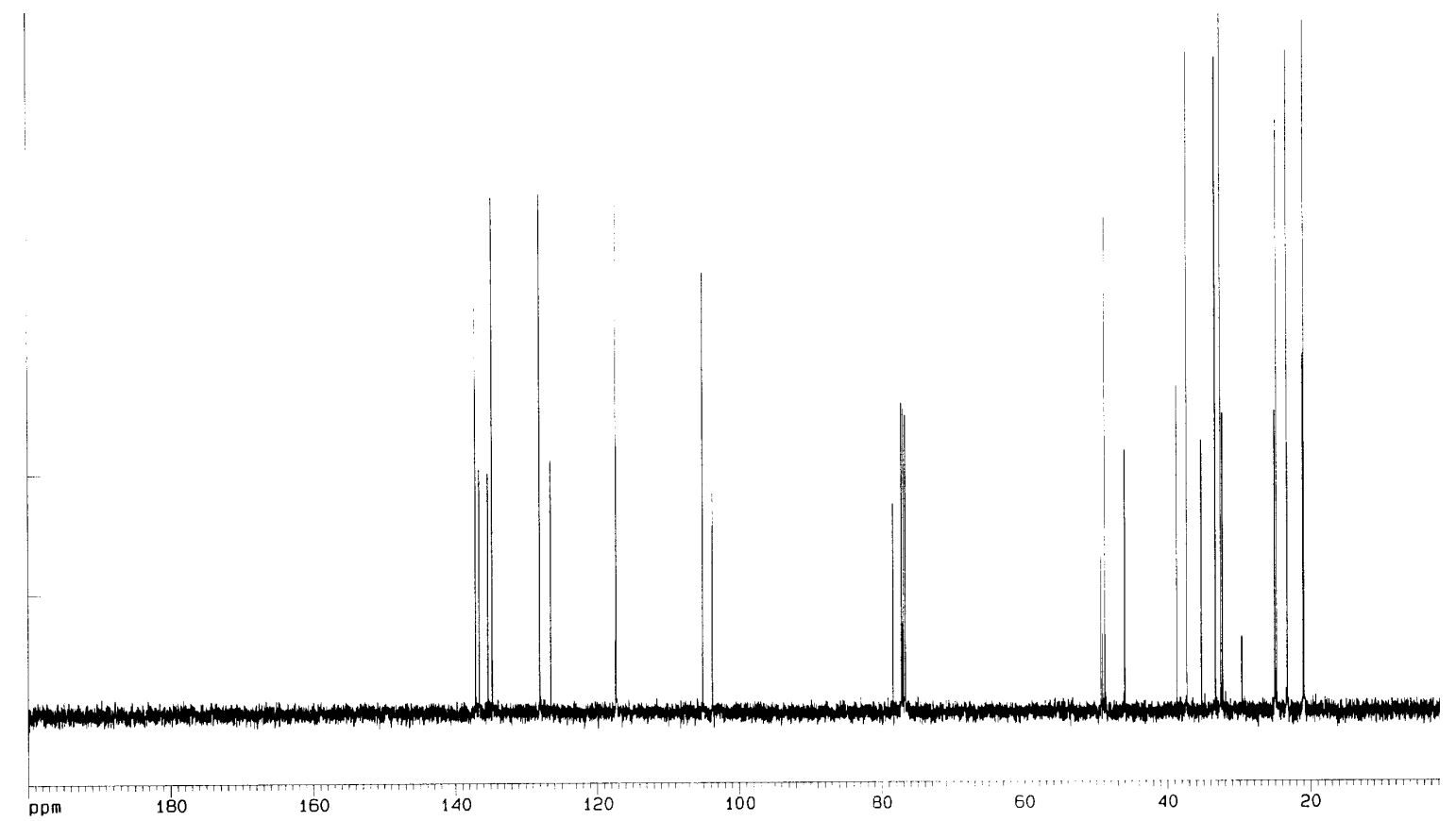




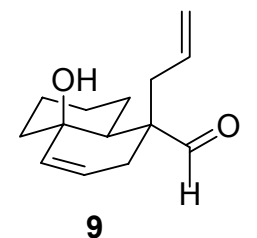

${ }^{1} \mathbf{H}$ NMR $\left(\mathrm{CDCl}_{3}, 500 \mathrm{MHz}\right)$
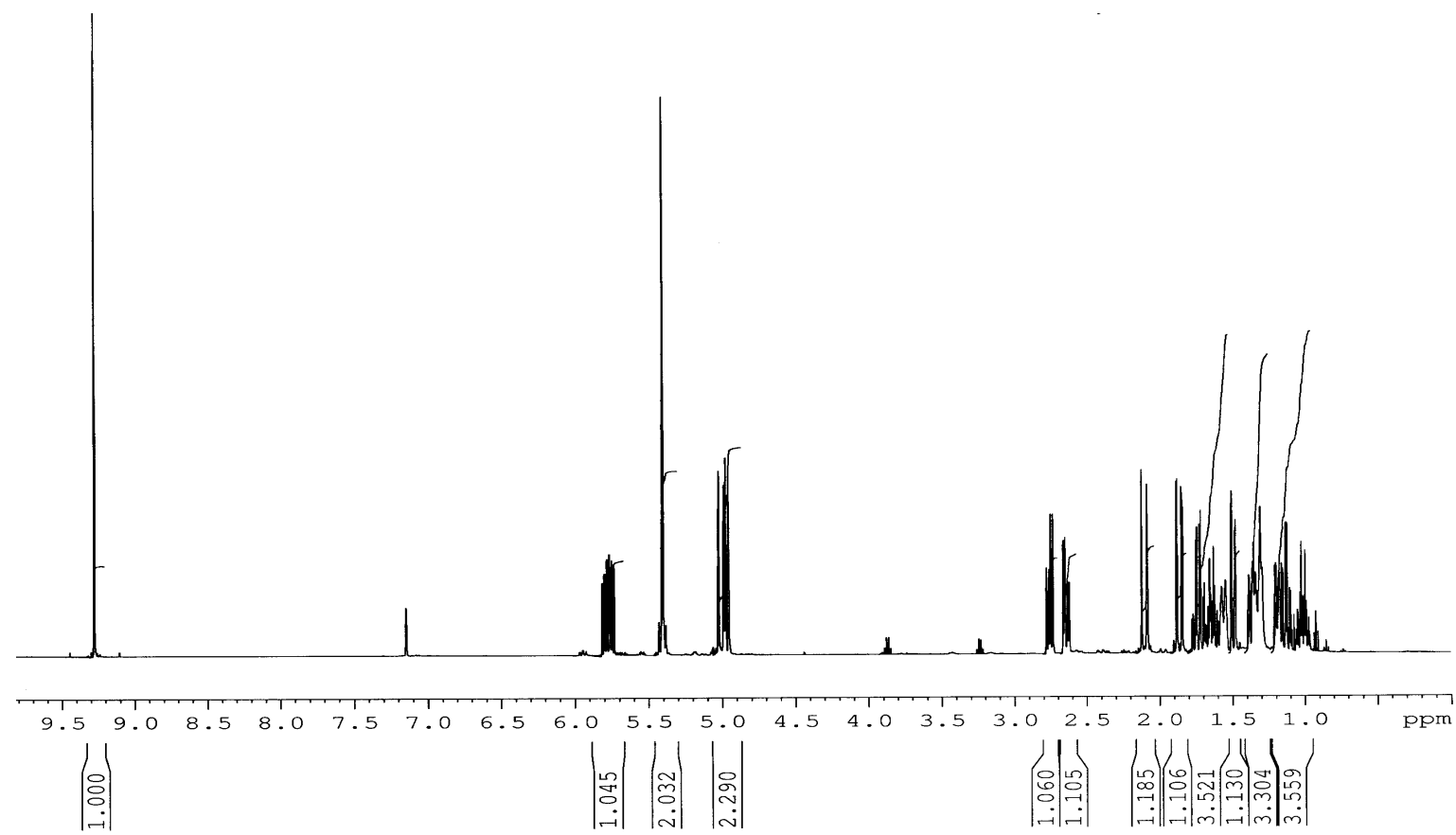

${ }^{13} \mathbf{C}$ NMR $\left(\mathrm{CDCl}_{3}, 125 \mathrm{MHz}\right)$

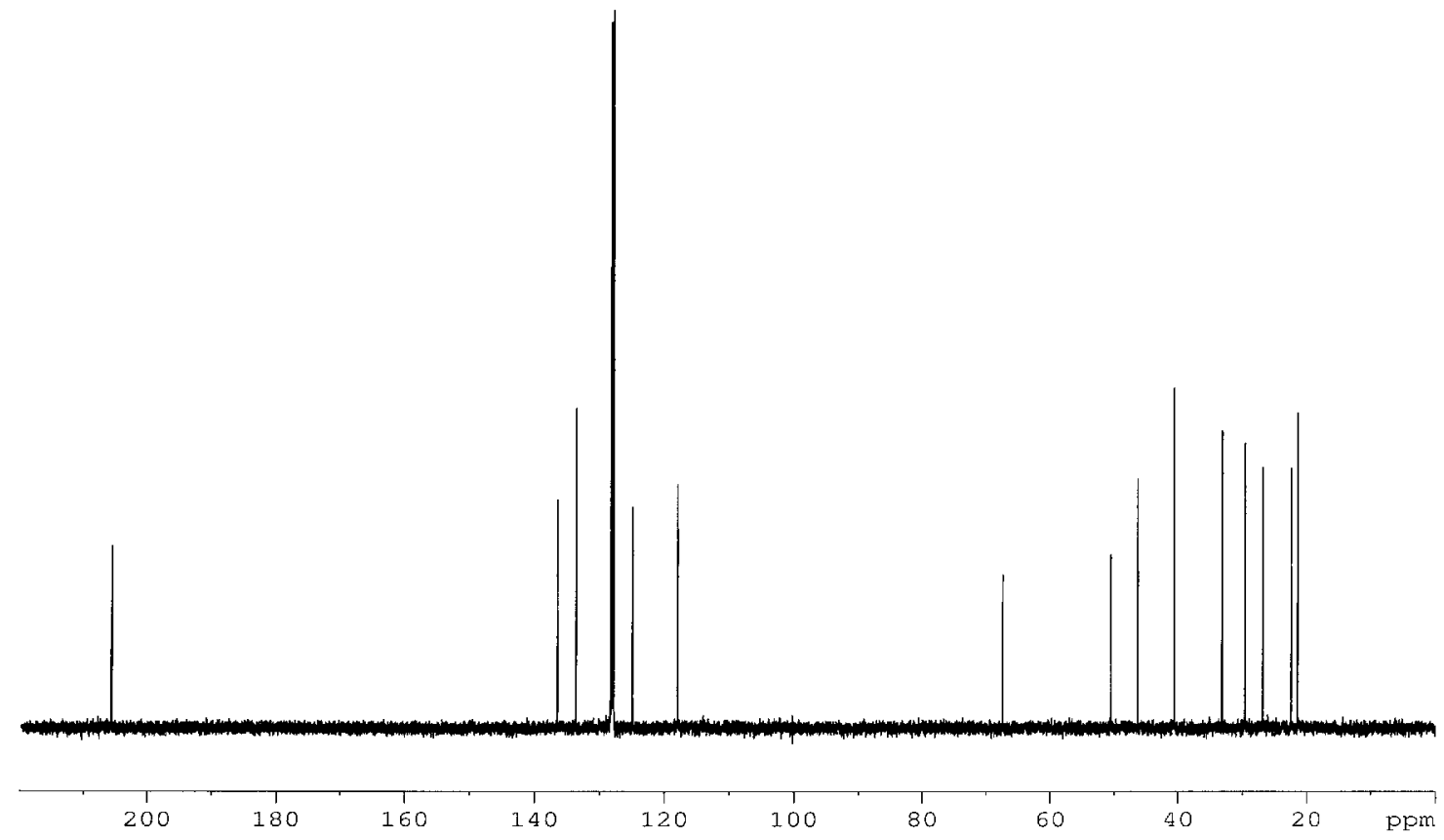




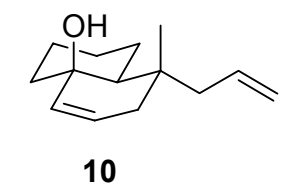

${ }^{1} \mathbf{H} \mathbf{N M R}\left(\mathrm{CDCl}_{3}, 500 \mathrm{MHz}\right)$
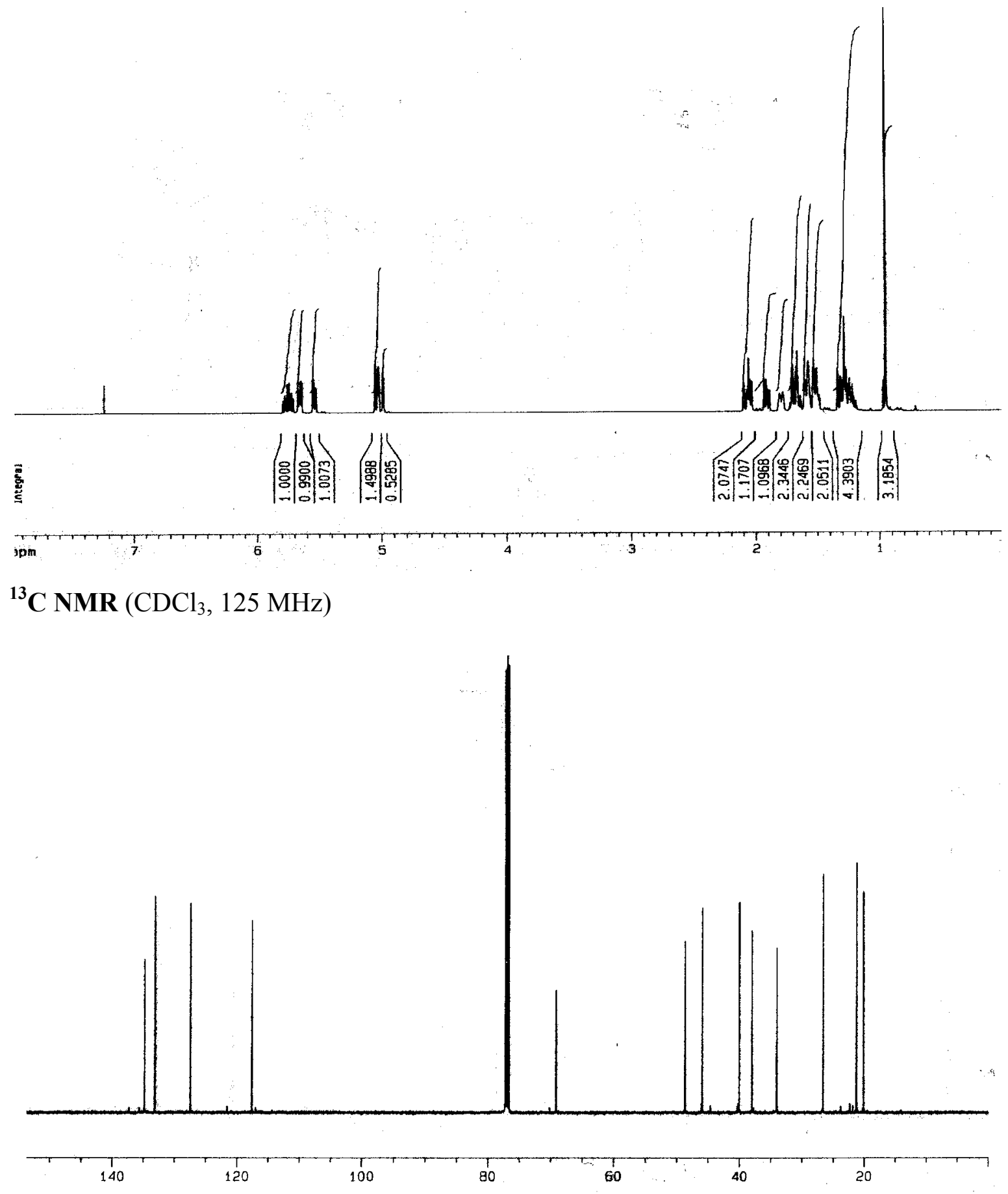


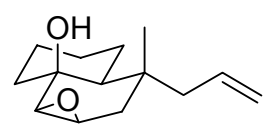

11

${ }^{1} \mathbf{H} \mathbf{N M R}\left(\mathrm{CDCl}_{3}, 300 \mathrm{MHz}\right)$

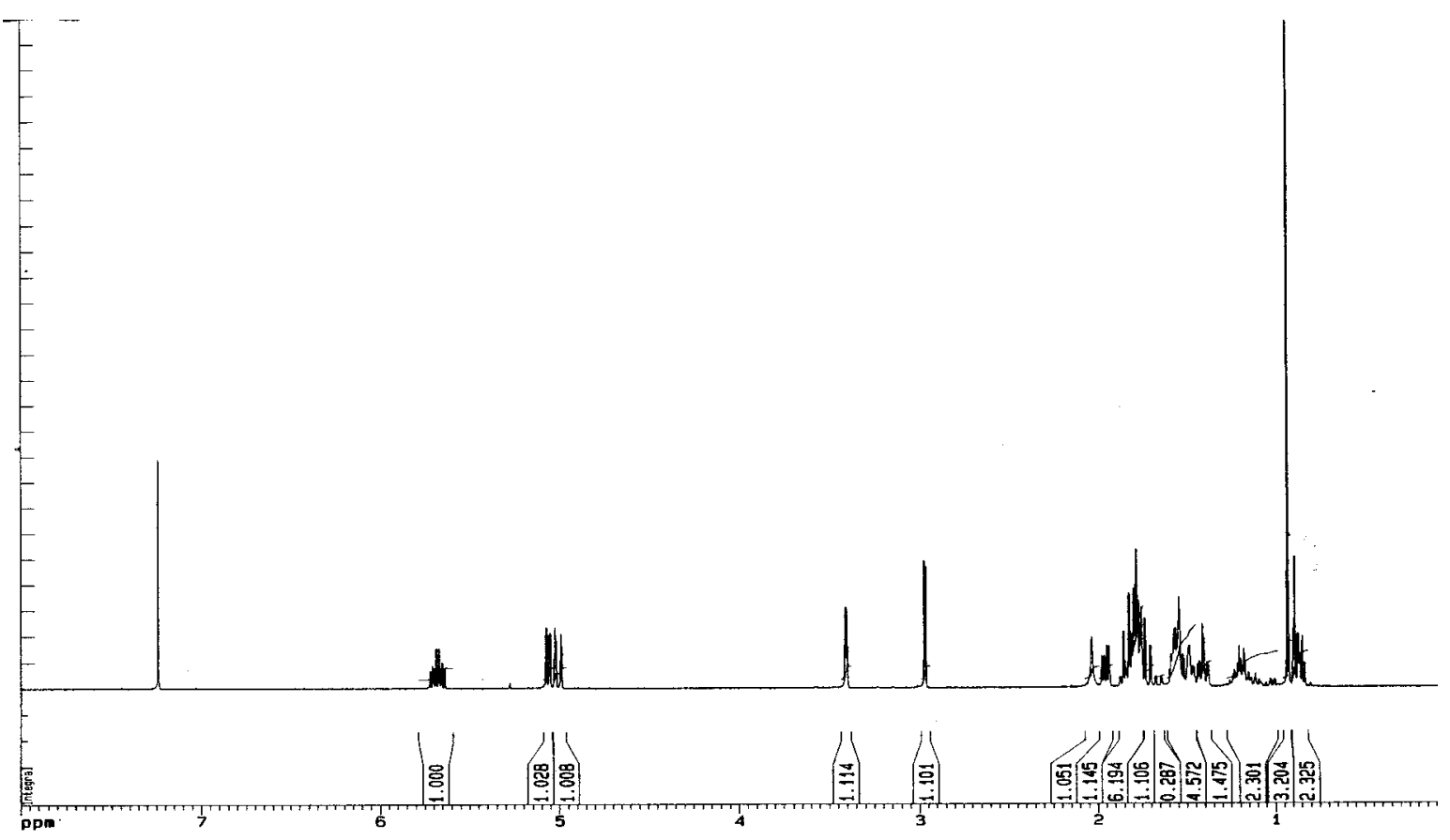

${ }^{13} \mathbf{C}$ NMR $\left(\mathrm{CDCl}_{3}, 75 \mathrm{MHz}\right)$

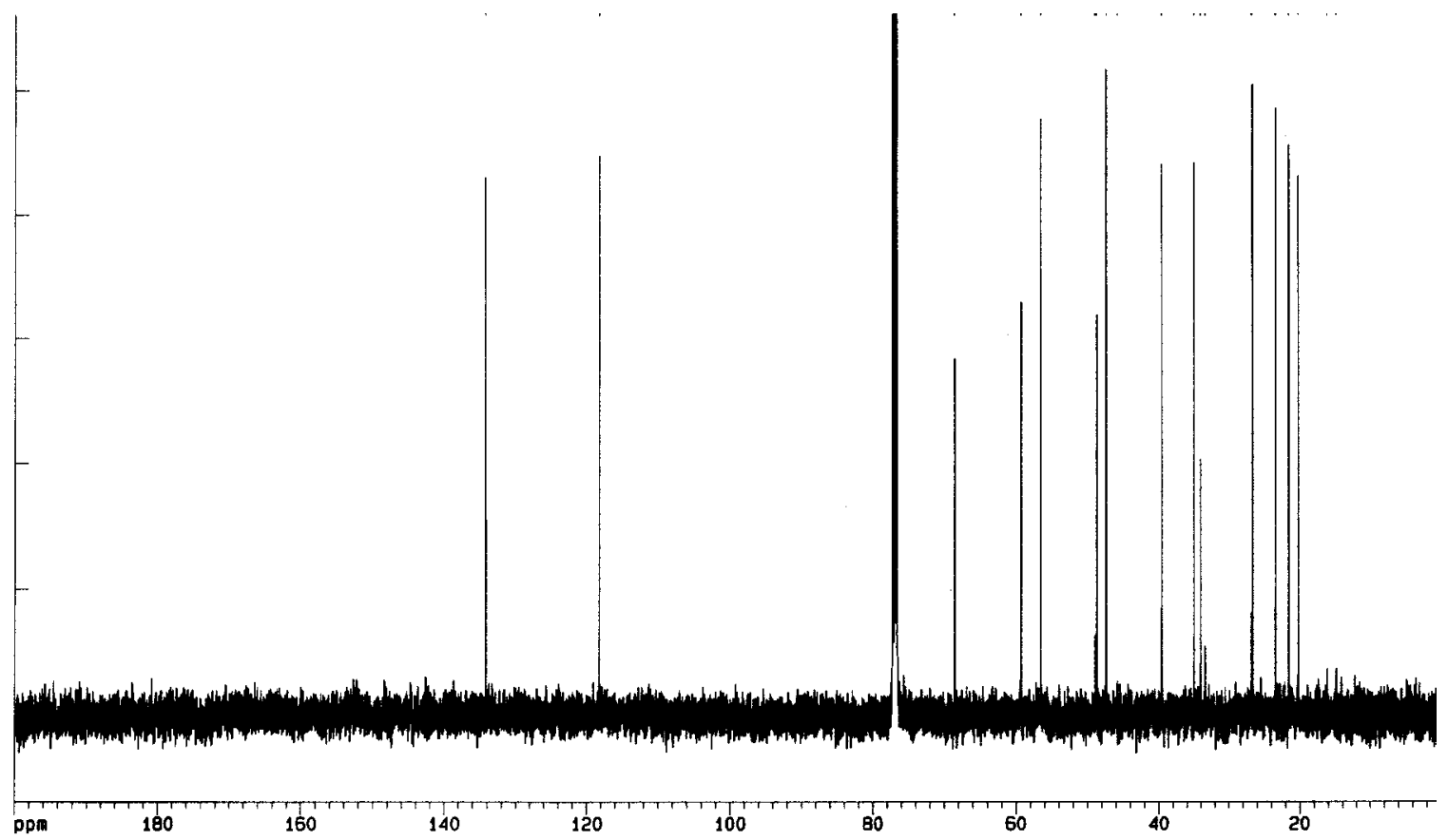




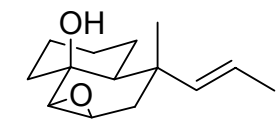

12

${ }^{1} \mathbf{H}$ NMR $\left(\mathrm{CDCl}_{3}, 500 \mathrm{MHz}\right)$

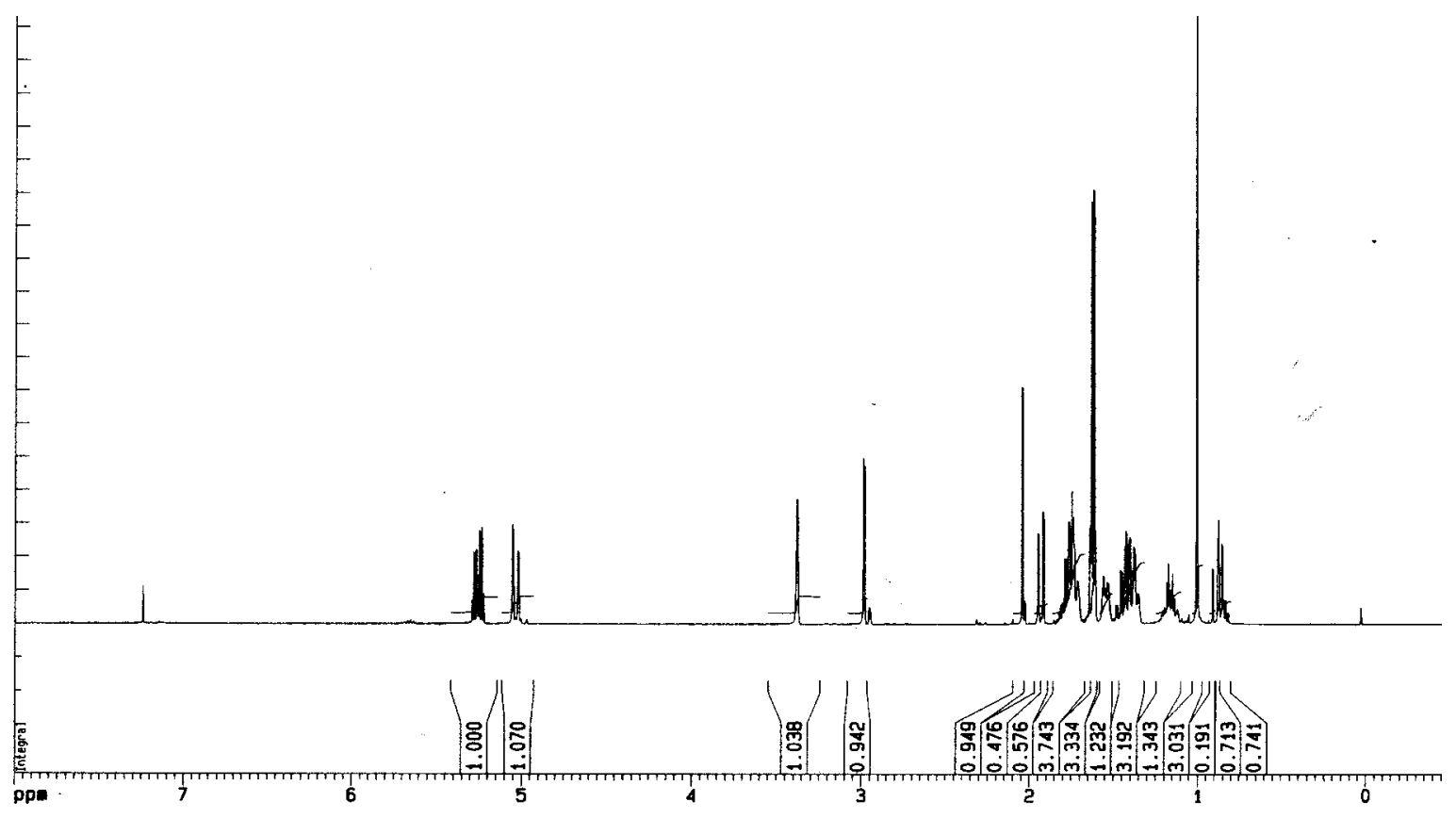

${ }^{13} \mathbf{C} \mathbf{N M R}\left(\mathrm{CDCl}_{3}, 125 \mathrm{MHz}\right)$

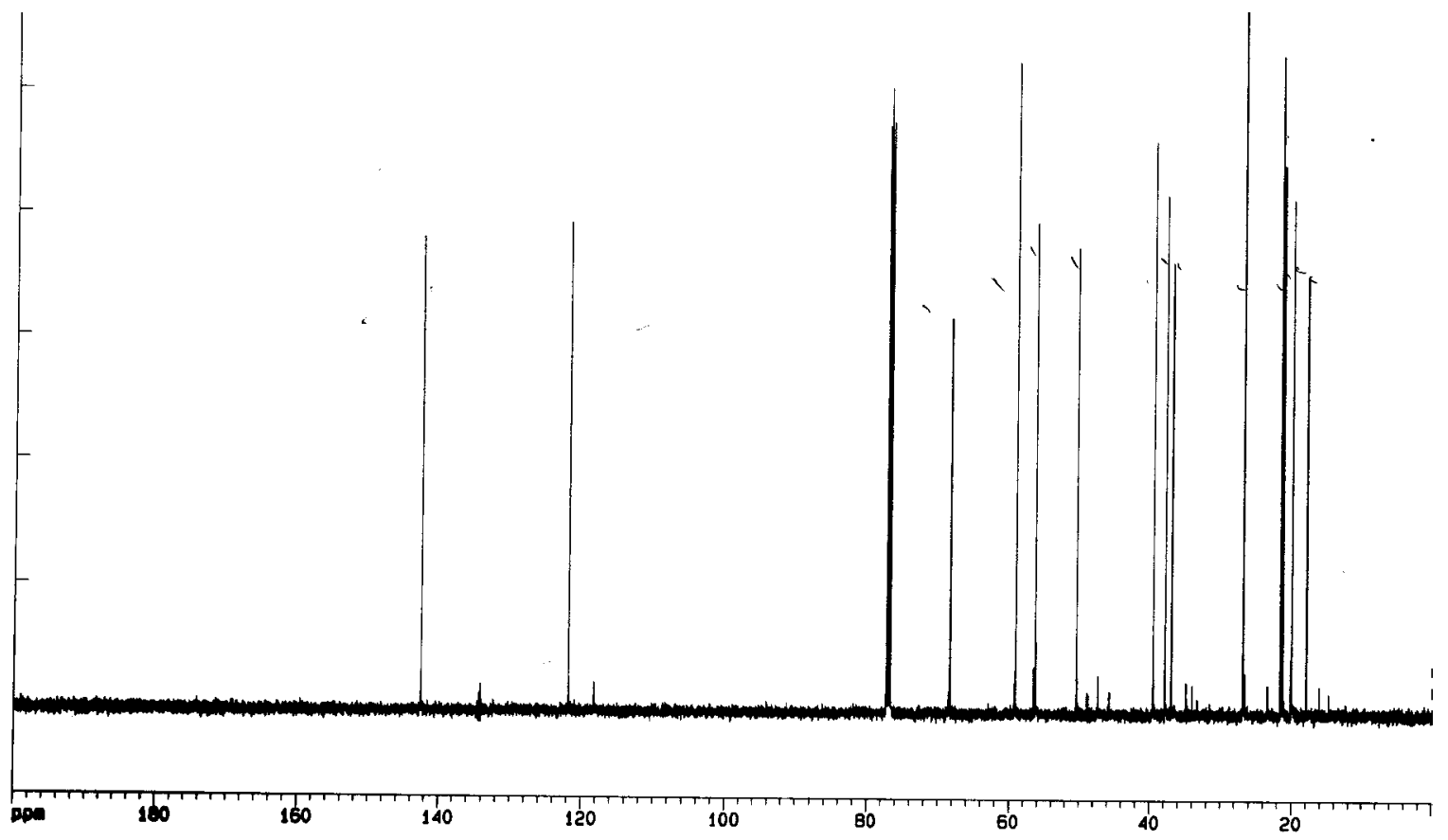




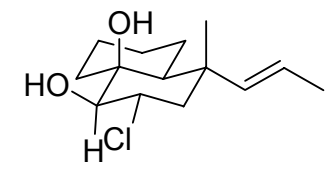

13

${ }^{\mathbf{1}} \mathbf{H} \mathbf{N M R}\left(\mathrm{CDCl}_{3}, 500 \mathrm{MHz}\right)$

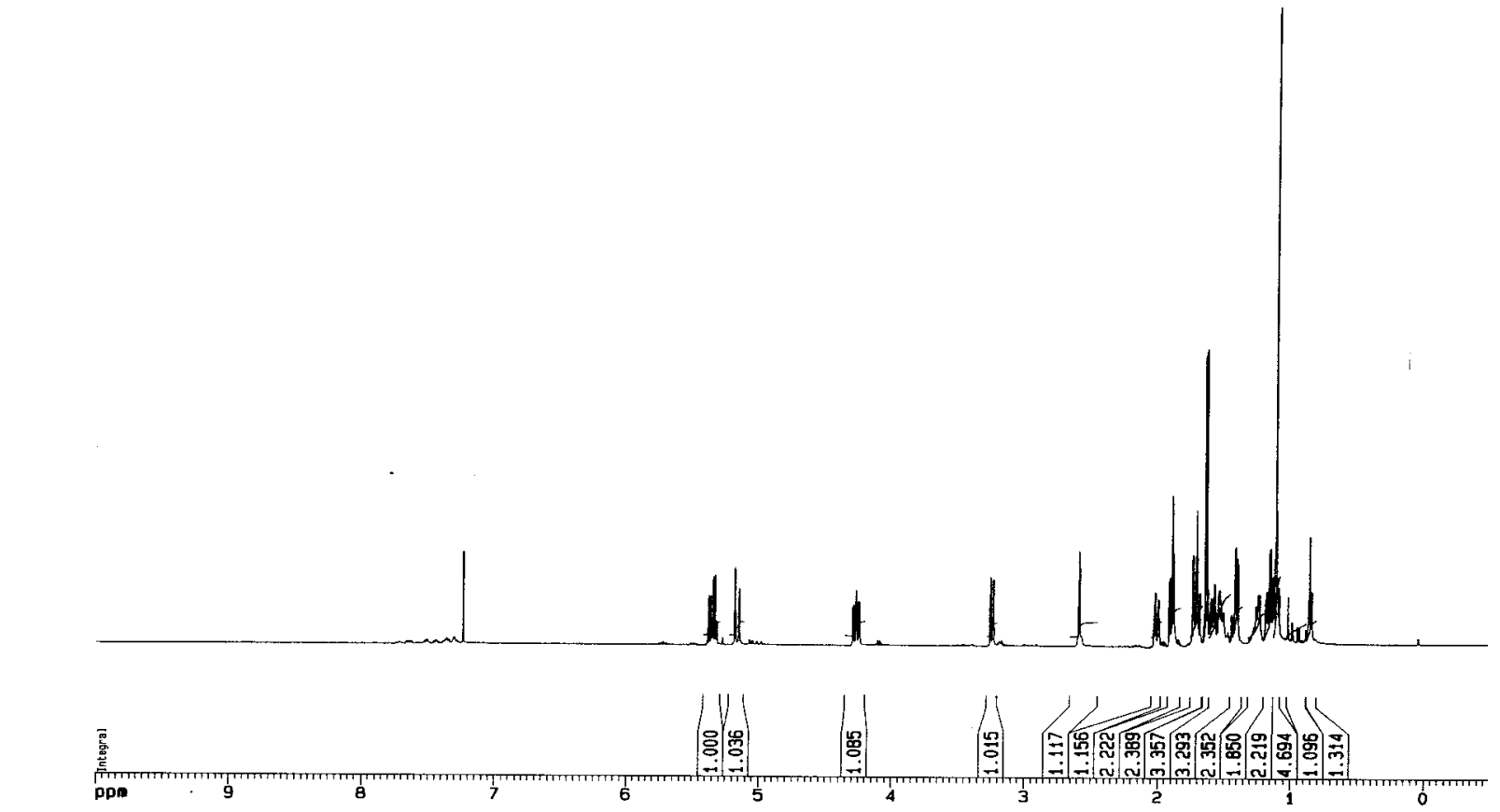

${ }^{13} \mathbf{C}$ NMR $\left(\mathrm{CDCl}_{3}, 125 \mathrm{MHz}\right)$
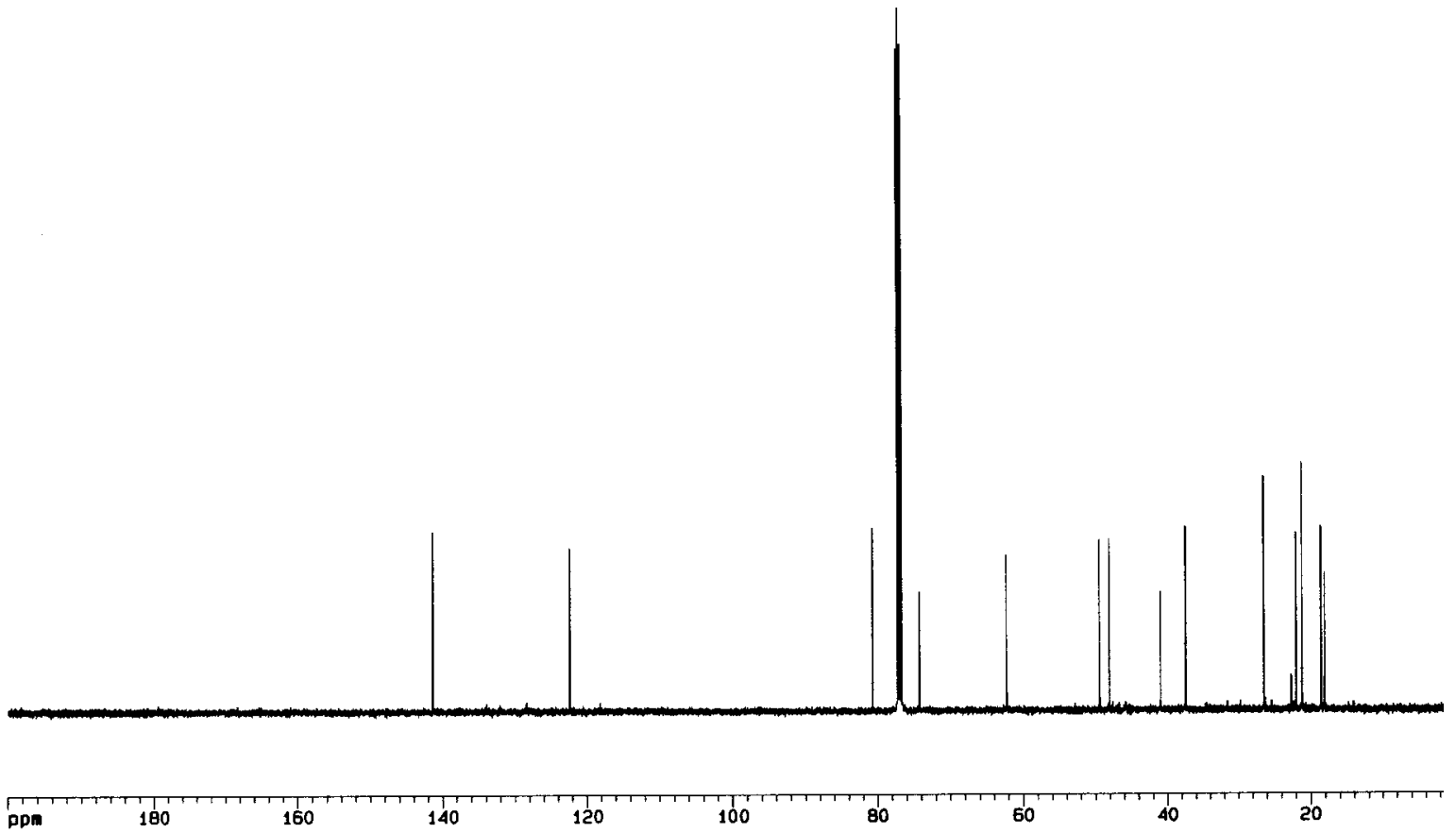


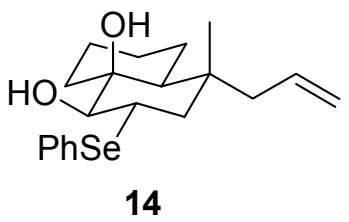

${ }^{1} \mathbf{H}$ NMR $\left(\mathrm{CDCl}_{3}, 500 \mathrm{MHz}\right)$

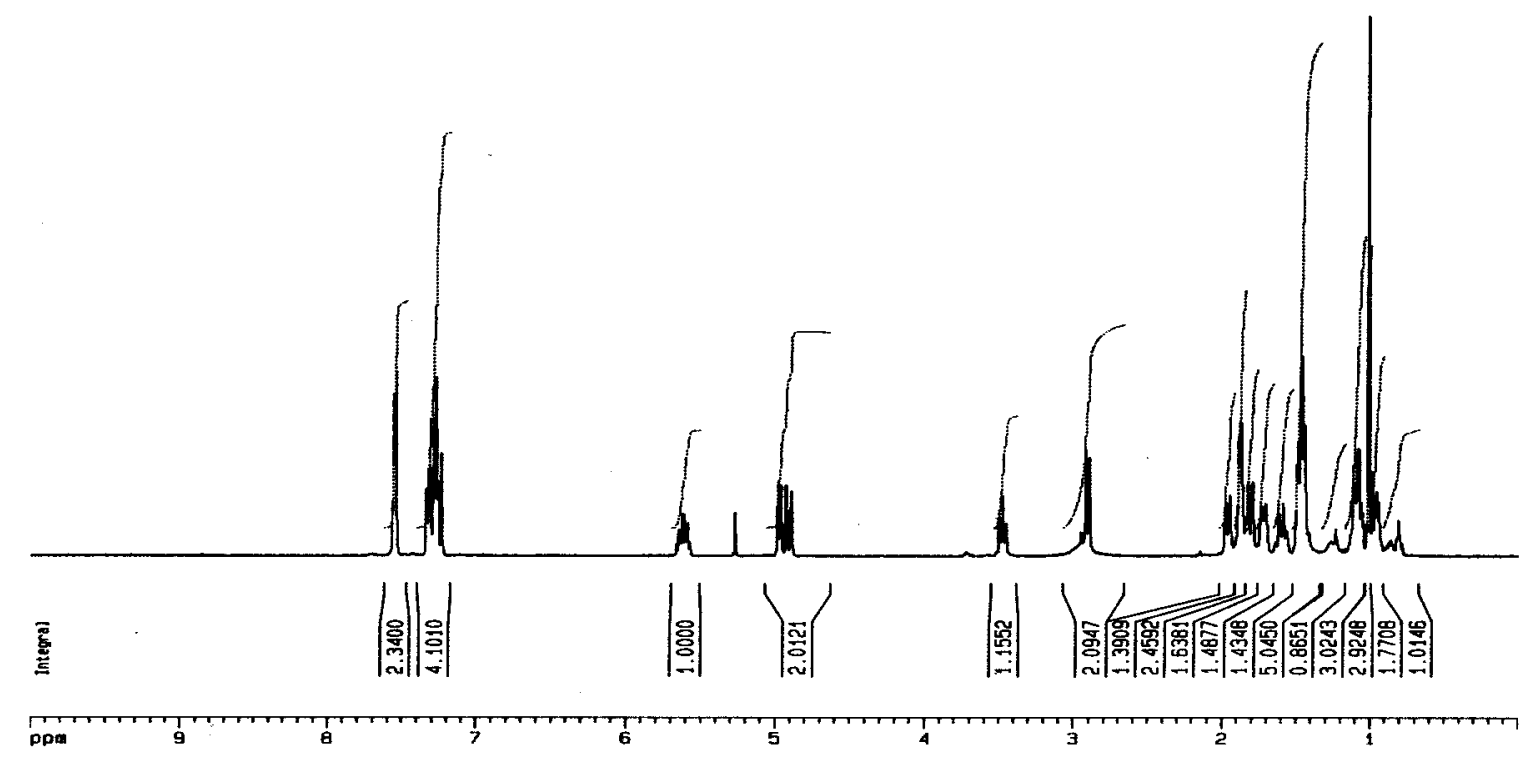

${ }^{13} \mathbf{C}$ NMR $\left(\mathrm{CDCl}_{3}, 125 \mathrm{MHz}\right)$

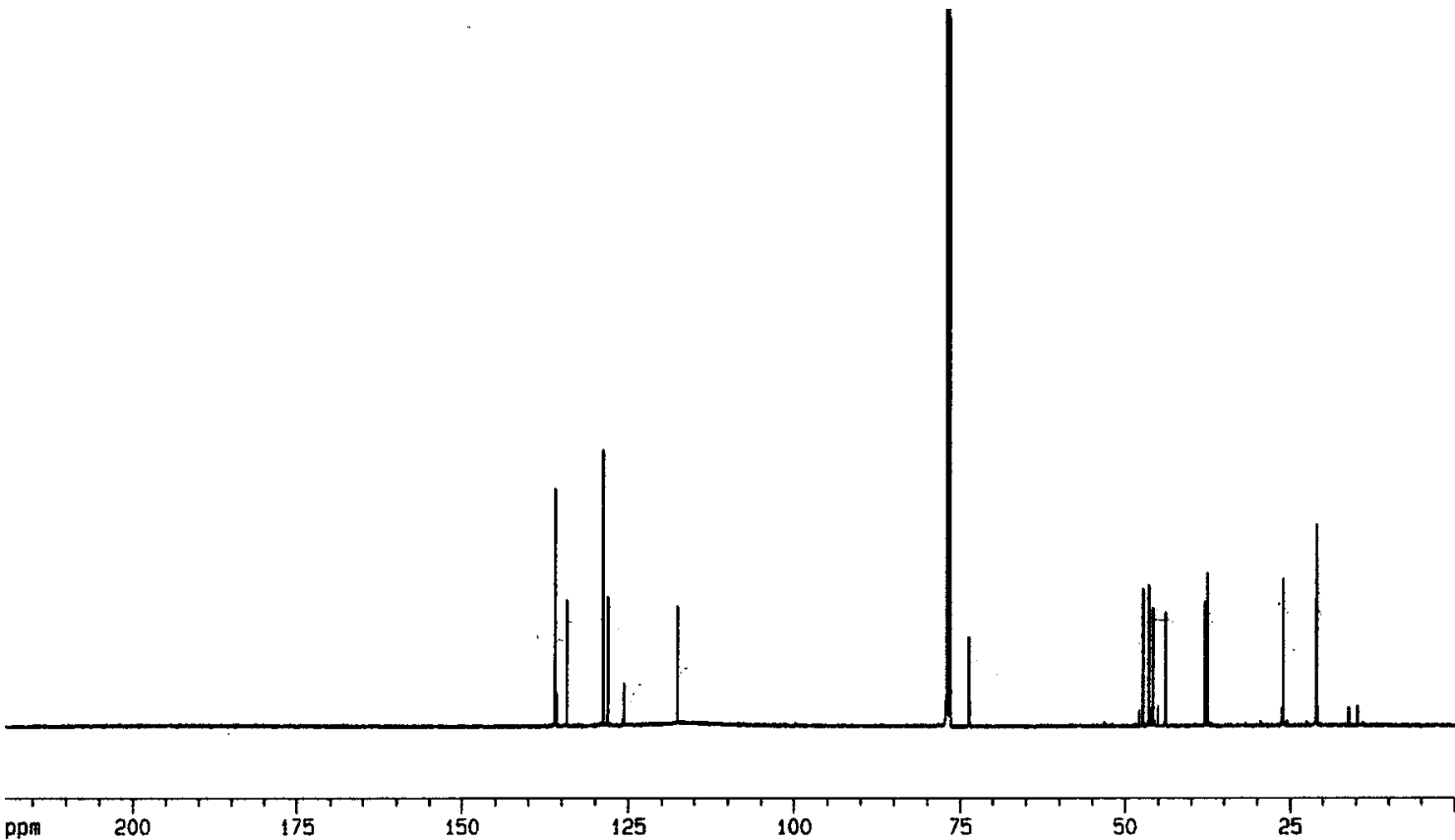




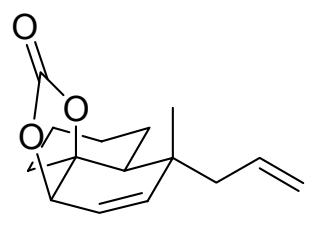

15

${ }^{1} \mathbf{H}$ NMR $\left(\mathrm{CDCl}_{3}, 500 \mathrm{MHz}\right)$

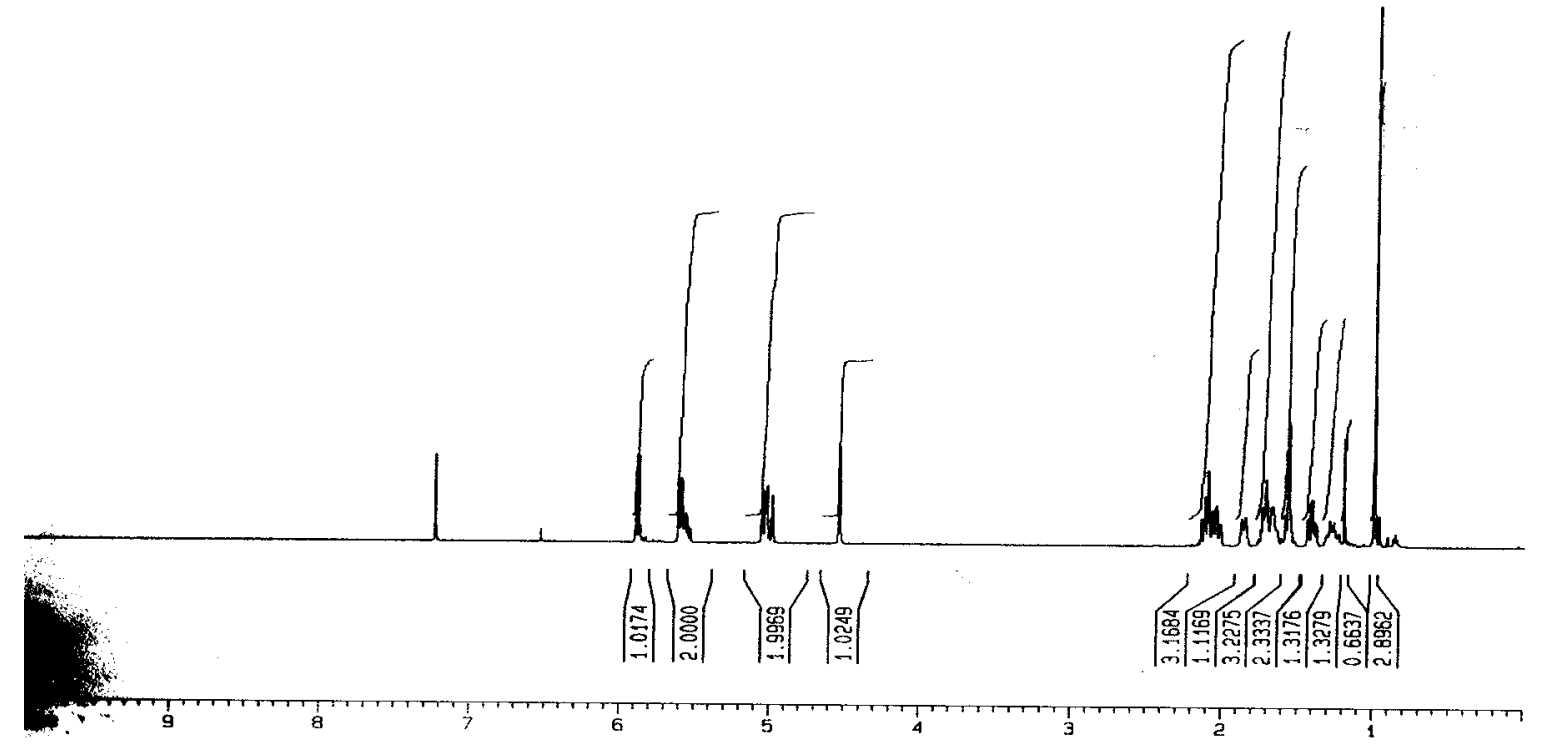

${ }^{13} \mathbf{C} \mathbf{N M R}\left(\mathrm{CDCl}_{3}, 125 \mathrm{MHz}\right)$

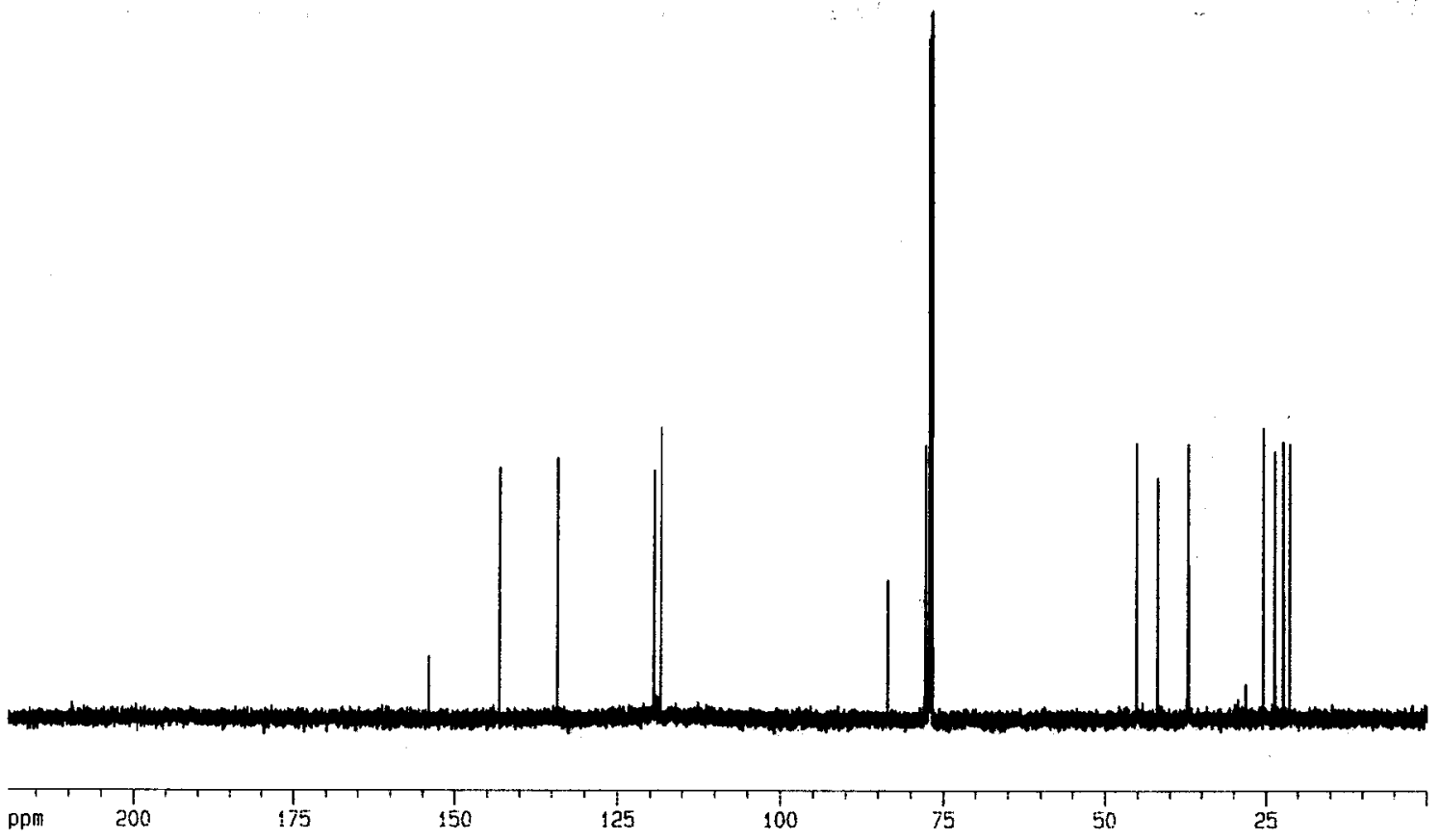




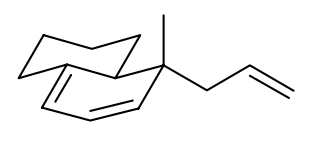

16

${ }^{\mathbf{1}} \mathbf{H} \mathbf{N M R}\left(\mathrm{CDCl}_{3}, 500 \mathrm{MHz}\right)$

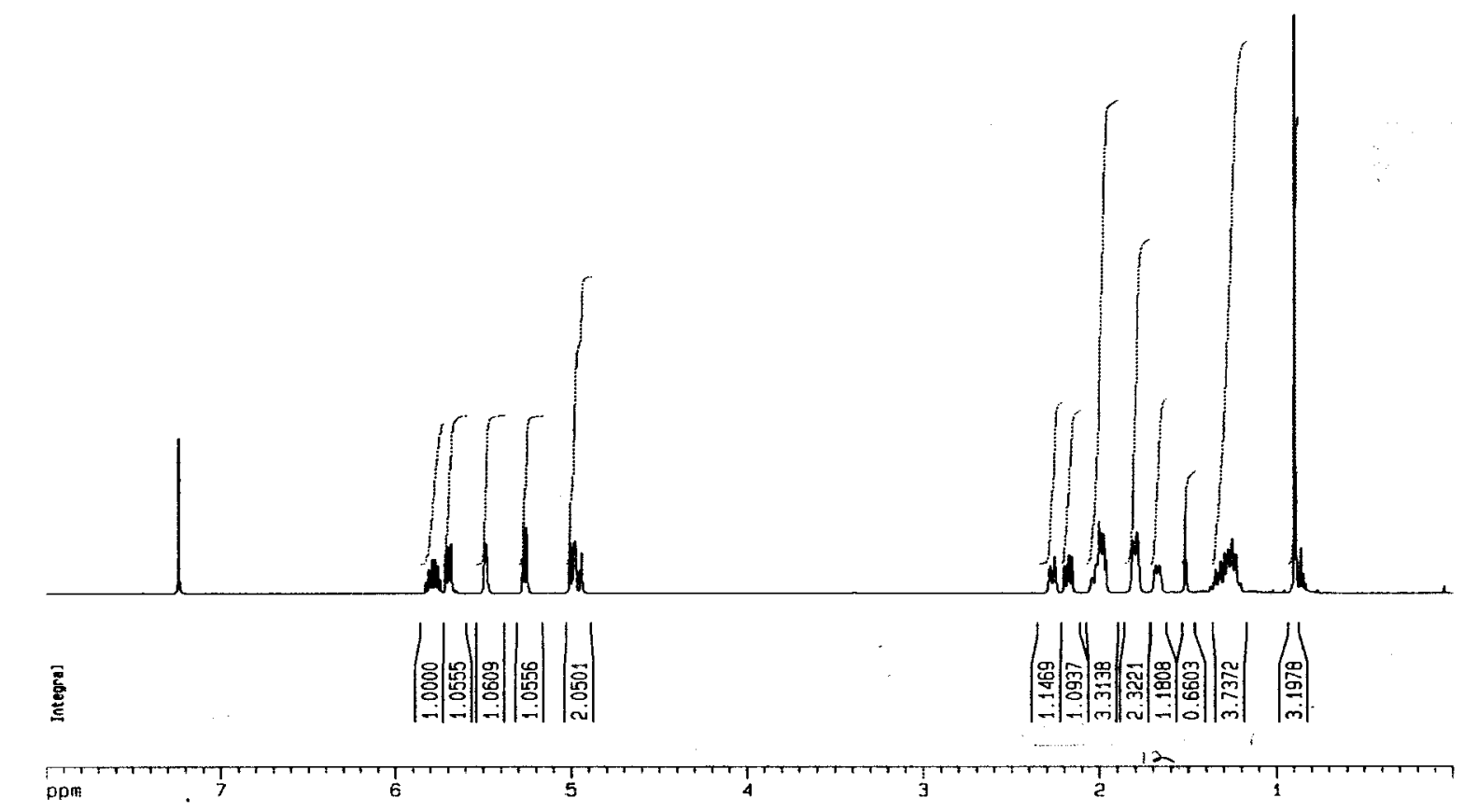

${ }^{13} \mathbf{C}$ NMR $\left(\mathrm{CDCl}_{3}, 125 \mathrm{MHz}\right)$

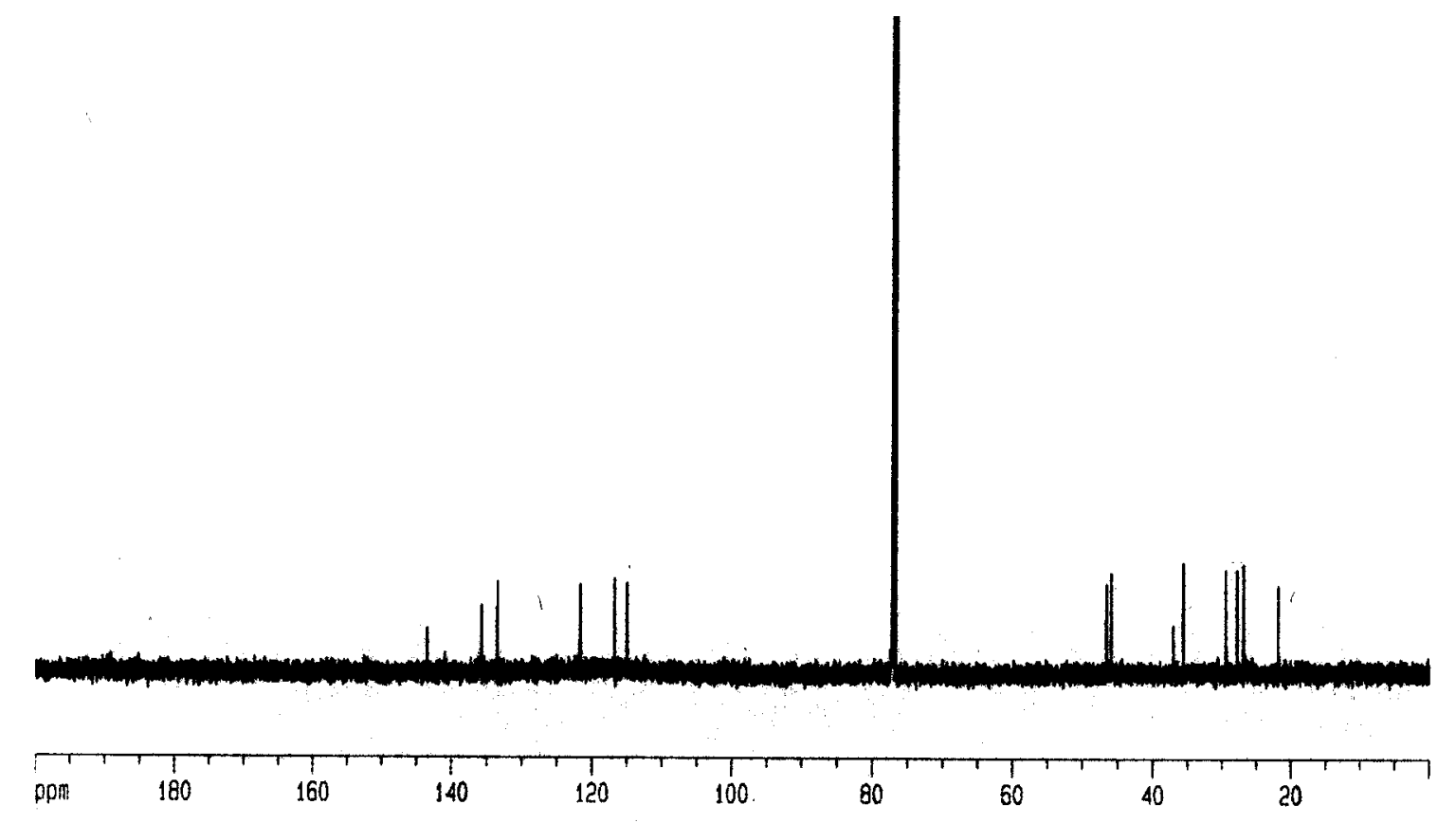




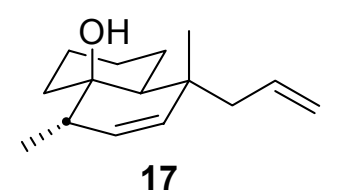

${ }^{1} \mathbf{H}$ NMR $\left(\mathrm{CDCl}_{3}, 500 \mathrm{MHz}\right)$

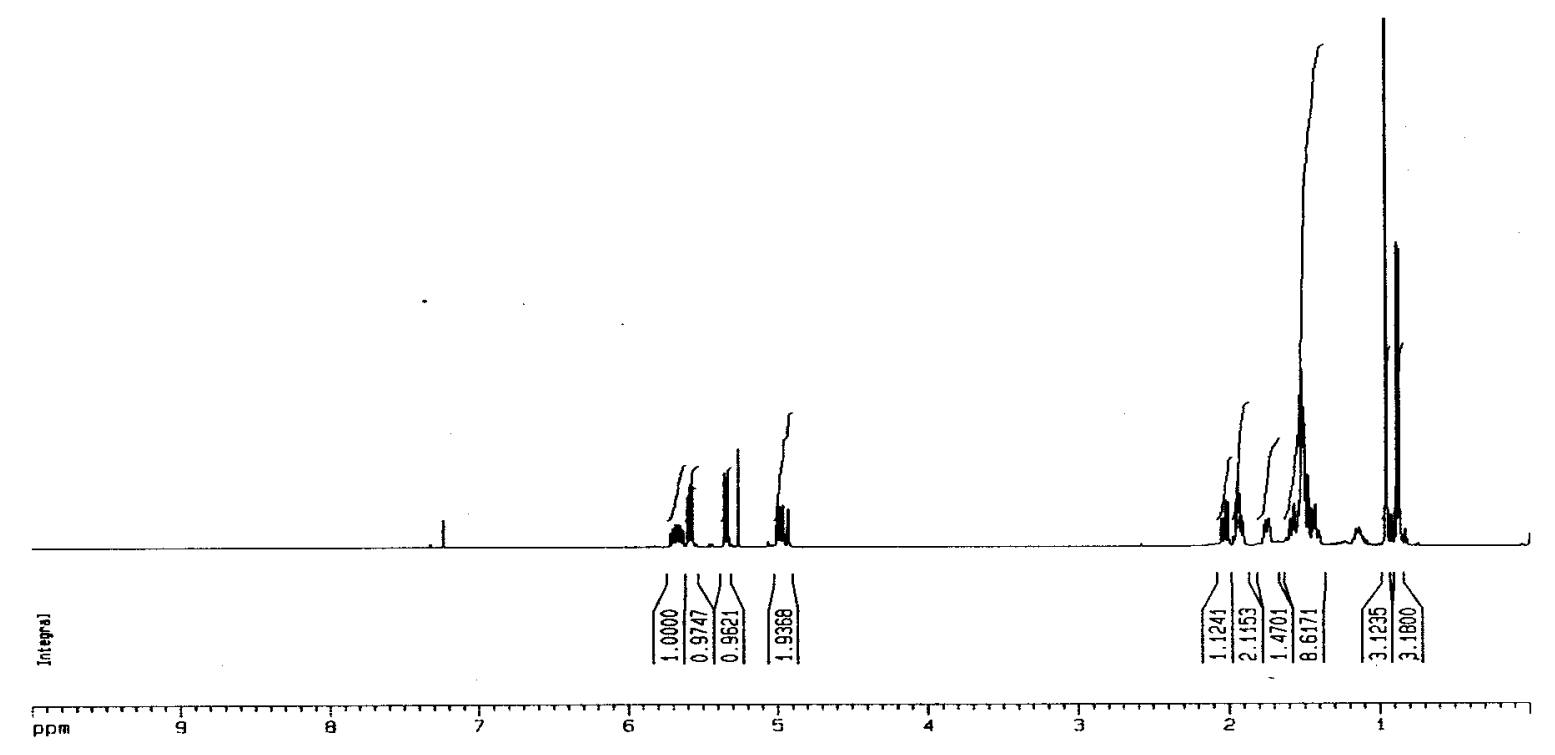

${ }^{13} \mathbf{C}$ NMR $\left(\mathrm{CDCl}_{3}, 125 \mathrm{MHz}\right)$

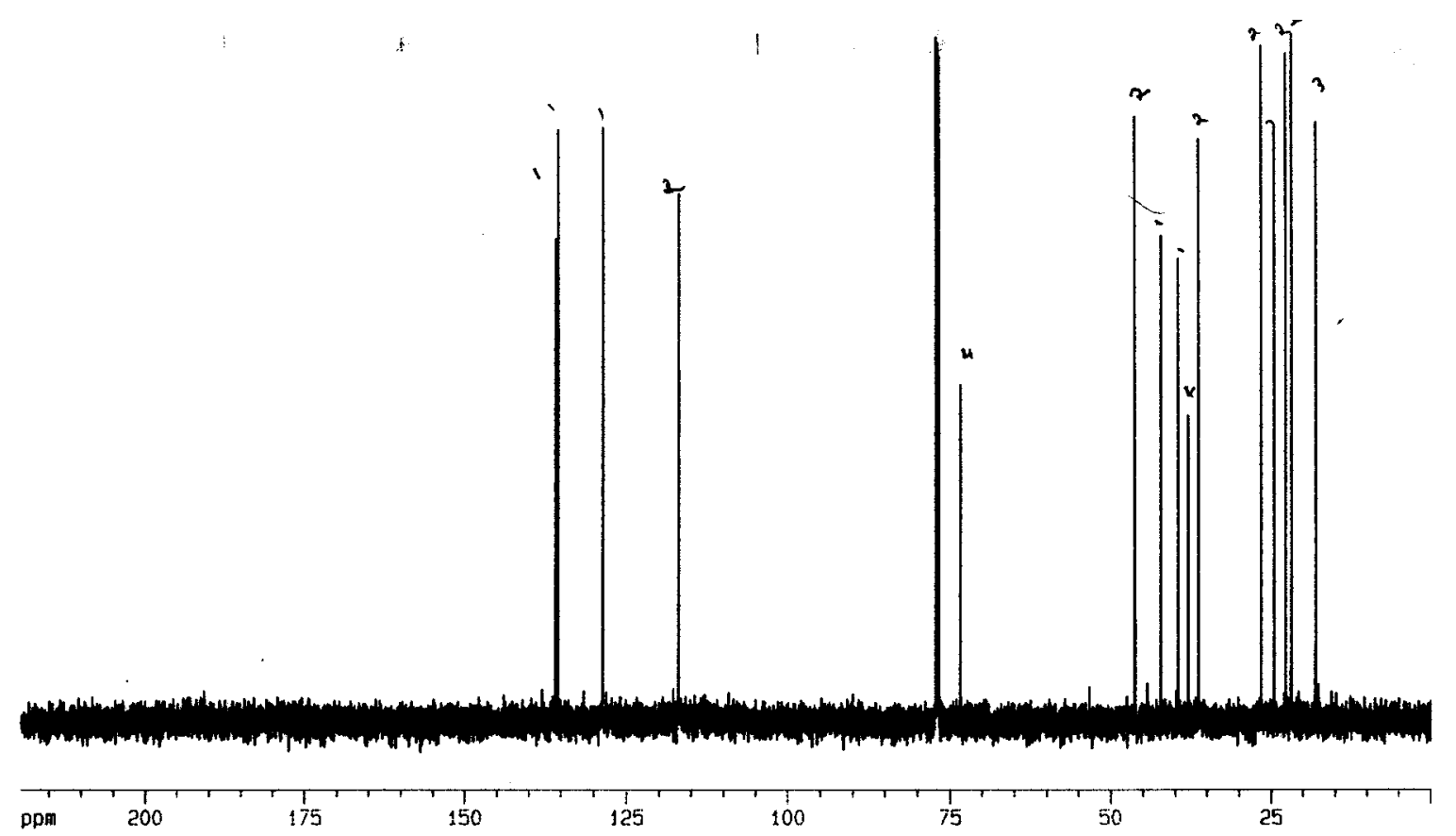




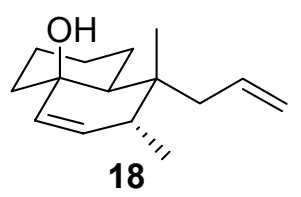

${ }^{1} \mathbf{H}$ NMR $\left(\mathrm{CDCl}_{3}, 500 \mathrm{MHz}\right)$

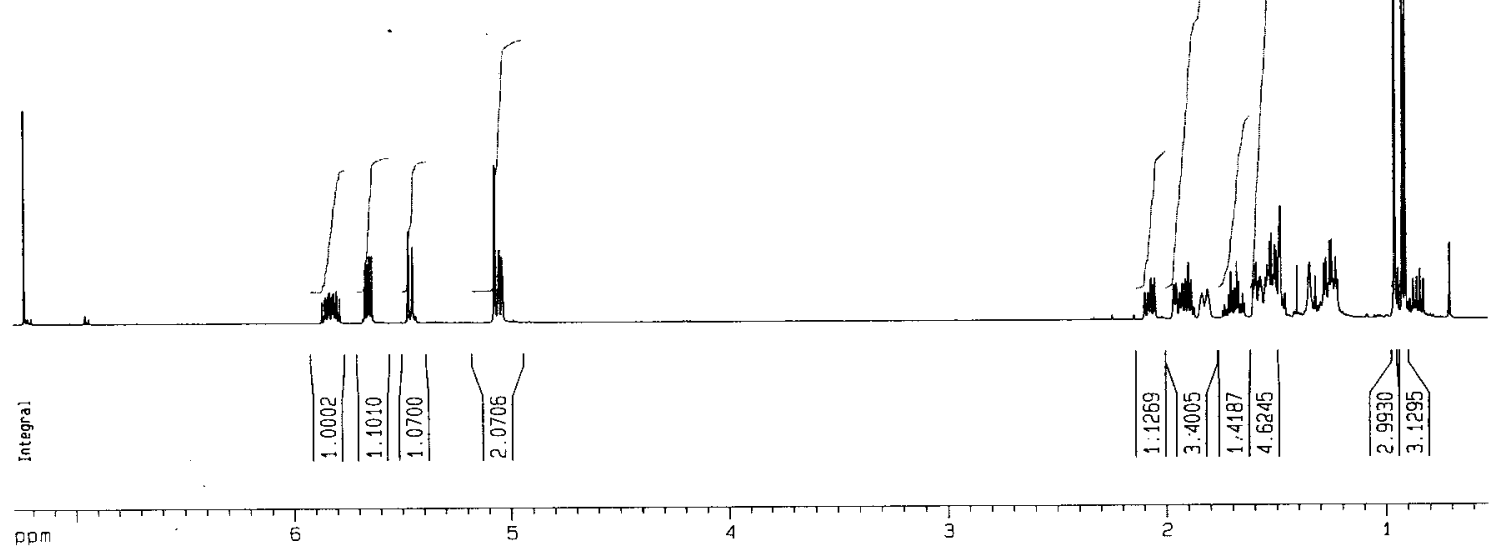

${ }^{13} \mathbf{C}$ NMR $\left(\mathrm{CDCl}_{3}, 125 \mathrm{MHz}\right)$

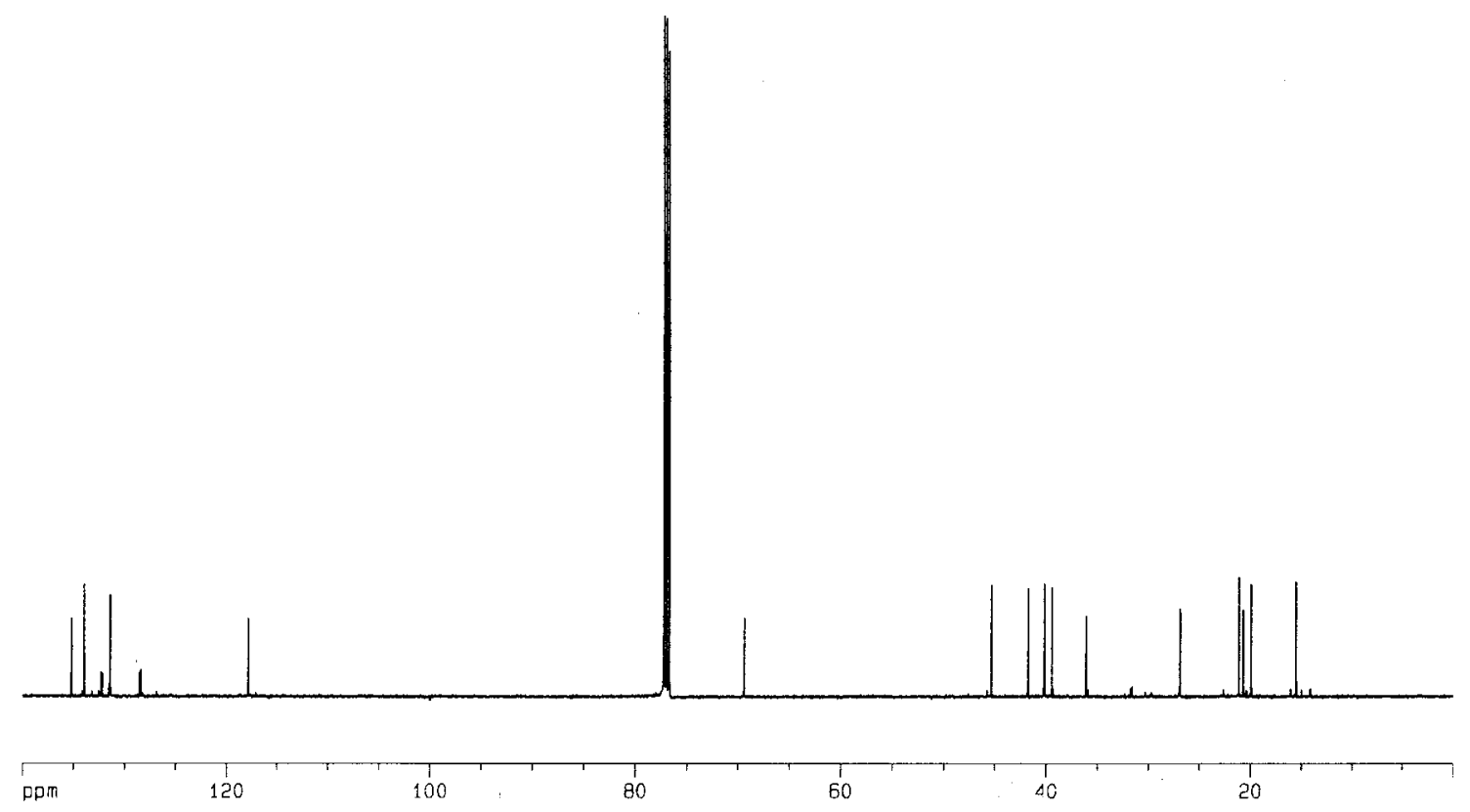

S34 


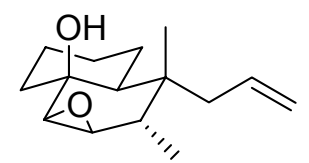

19

${ }^{1} \mathrm{H}$ NMR (CDCl 3 , 500 MHz)
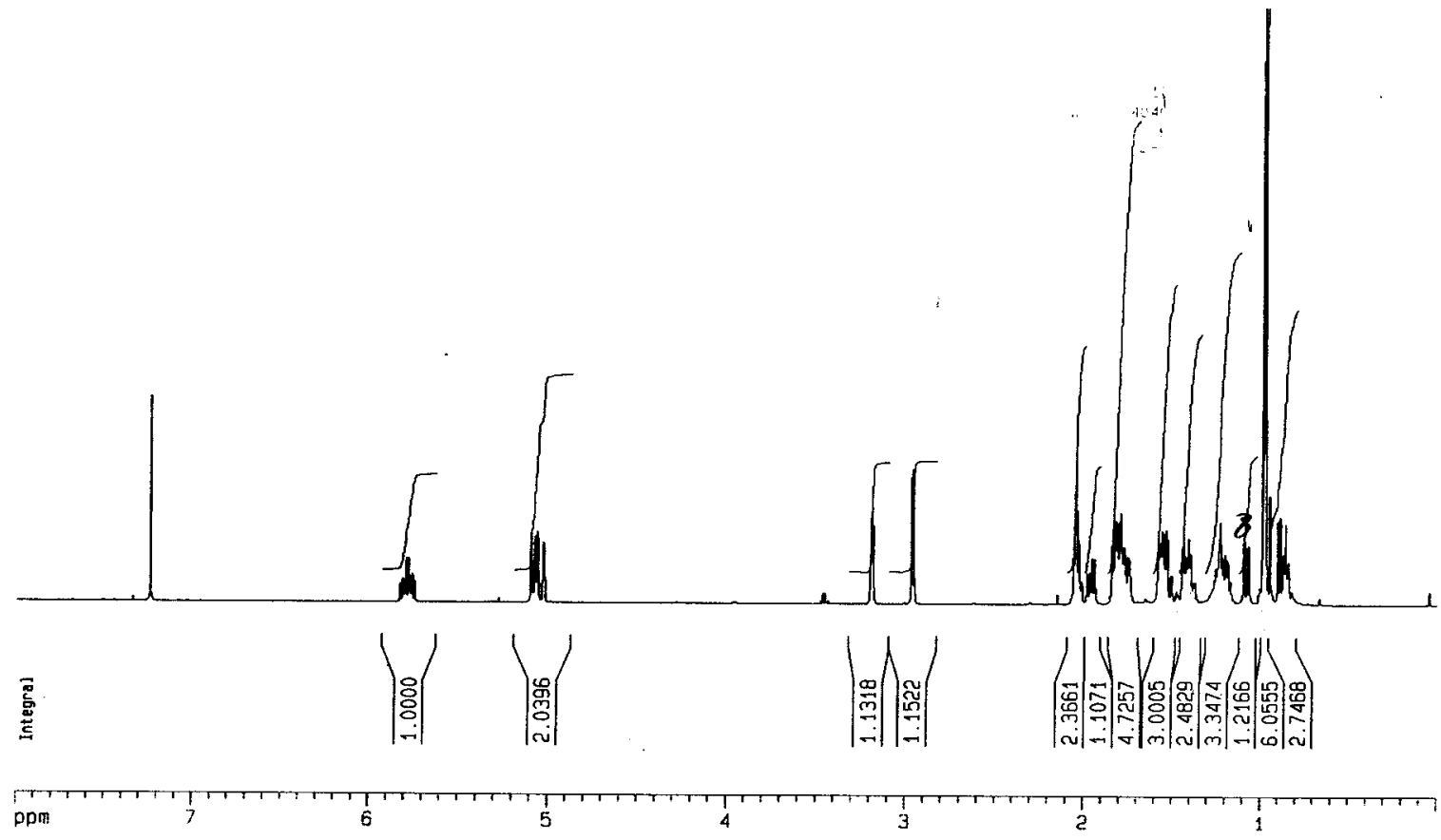

${ }^{13} \mathrm{C} \mathrm{NMR}\left(\mathrm{CDCl}_{\mathbf{3}}, \mathbf{1 2 5} \mathrm{MHz}\right)$

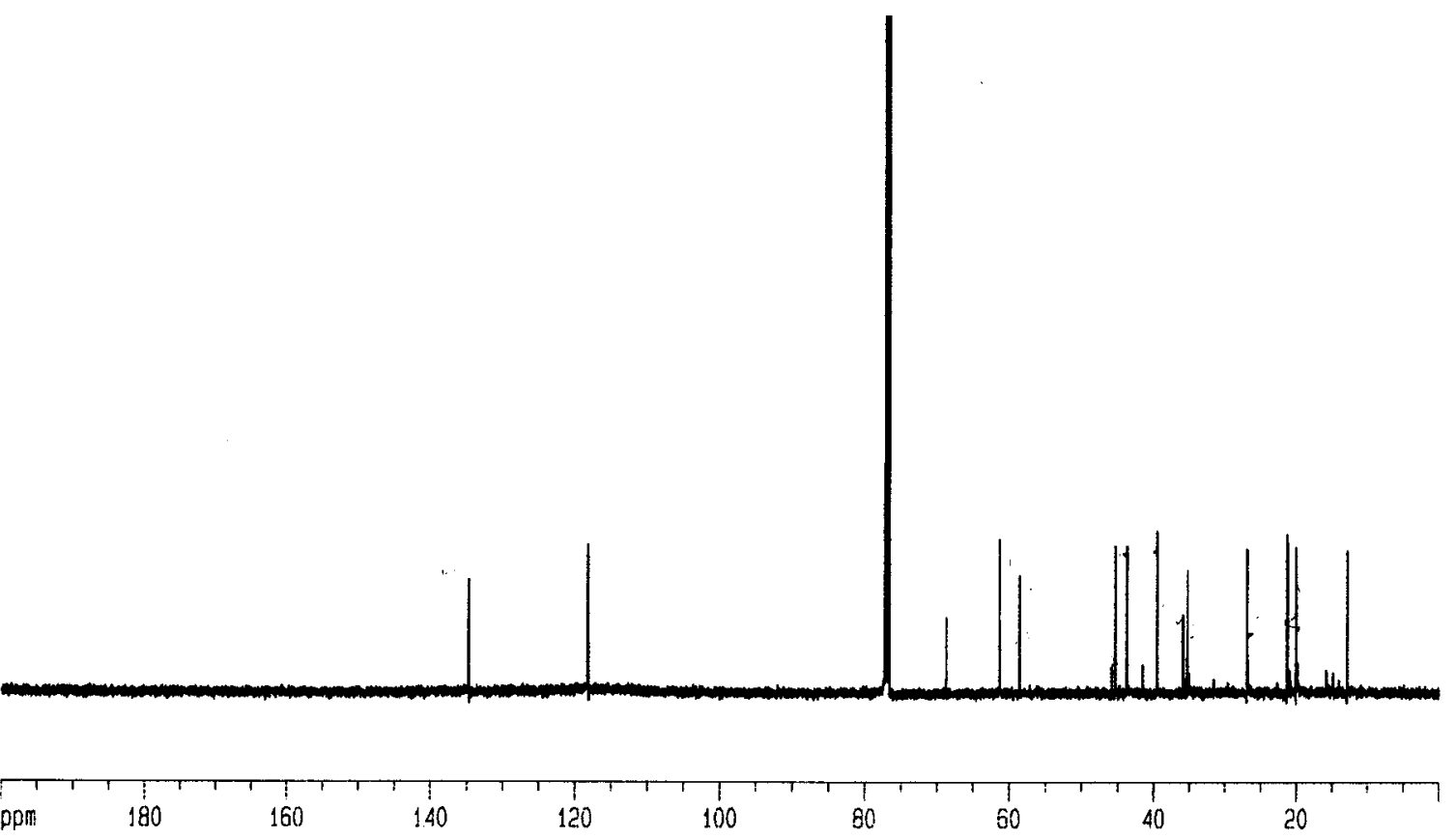




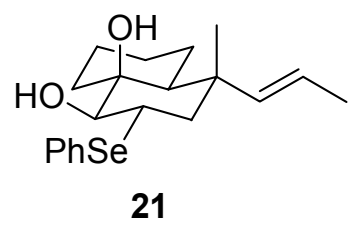

${ }^{1} \mathbf{H}$ NMR $\left(\mathrm{CDCl}_{3}, 500 \mathrm{MHz}\right)$

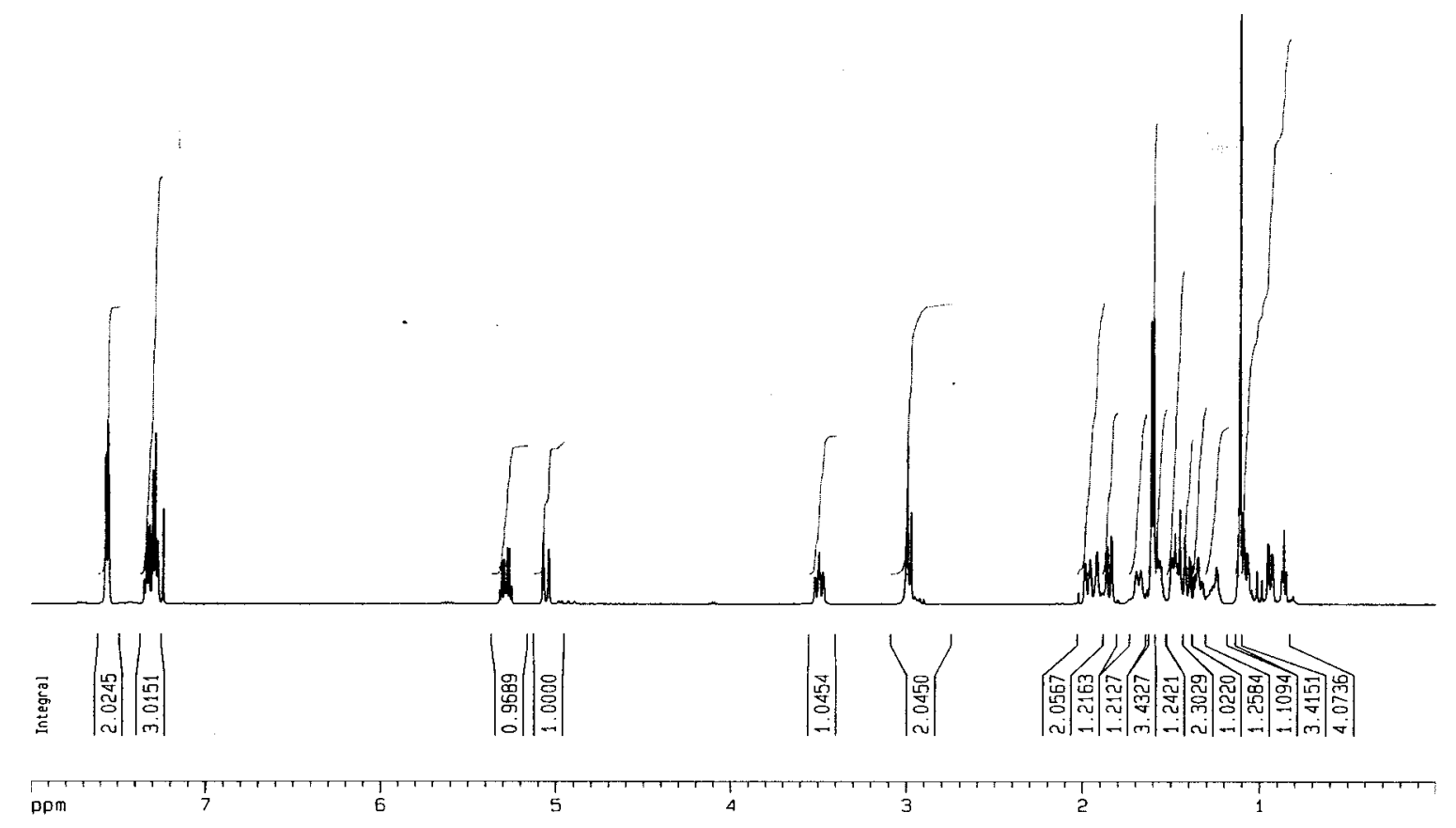

${ }^{13} \mathbf{C}$ NMR $\left(\mathrm{CDCl}_{3}, 125 \mathrm{MHz}\right)$

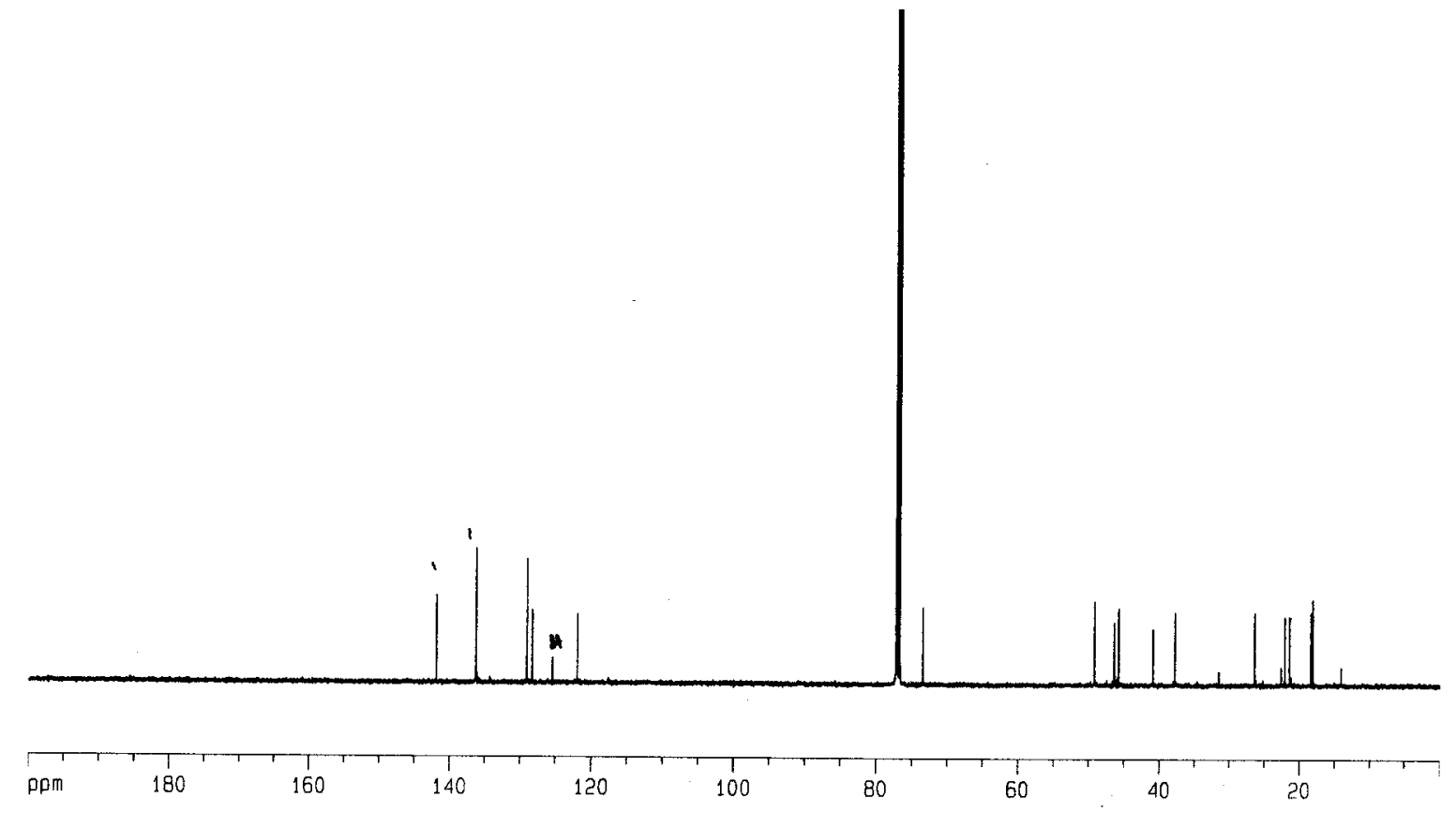




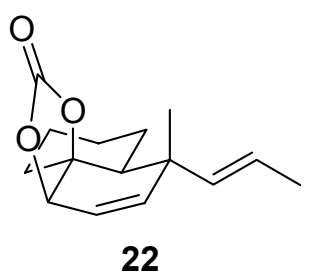

${ }^{1} \mathbf{H} \mathbf{N M R}\left(\mathrm{CDCl}_{3}, 500 \mathrm{MHz}\right)$

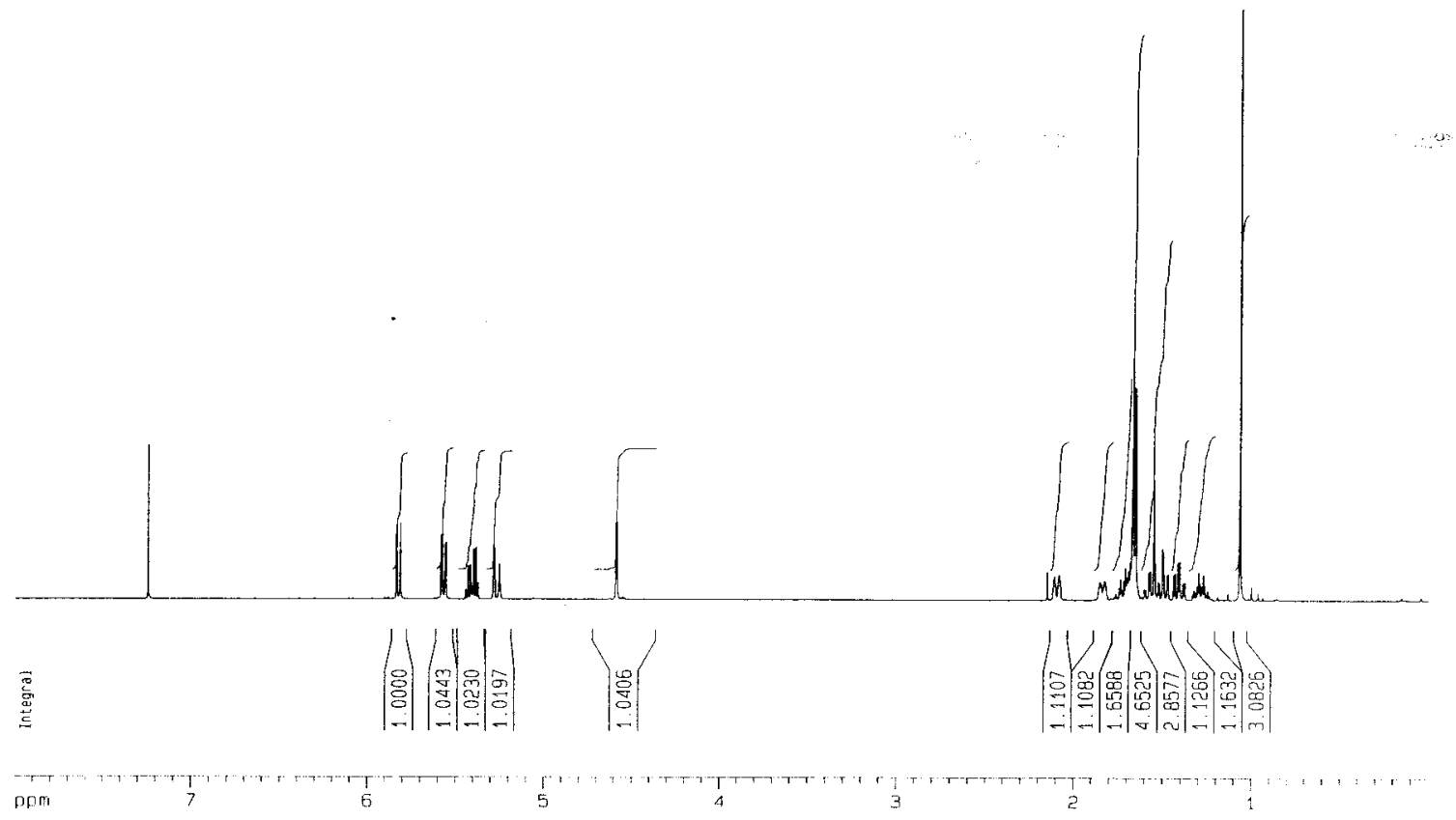

${ }^{13} \mathbf{C}$ NMR $\left(\mathrm{CDCl}_{3}, 125 \mathrm{MHz}\right)$
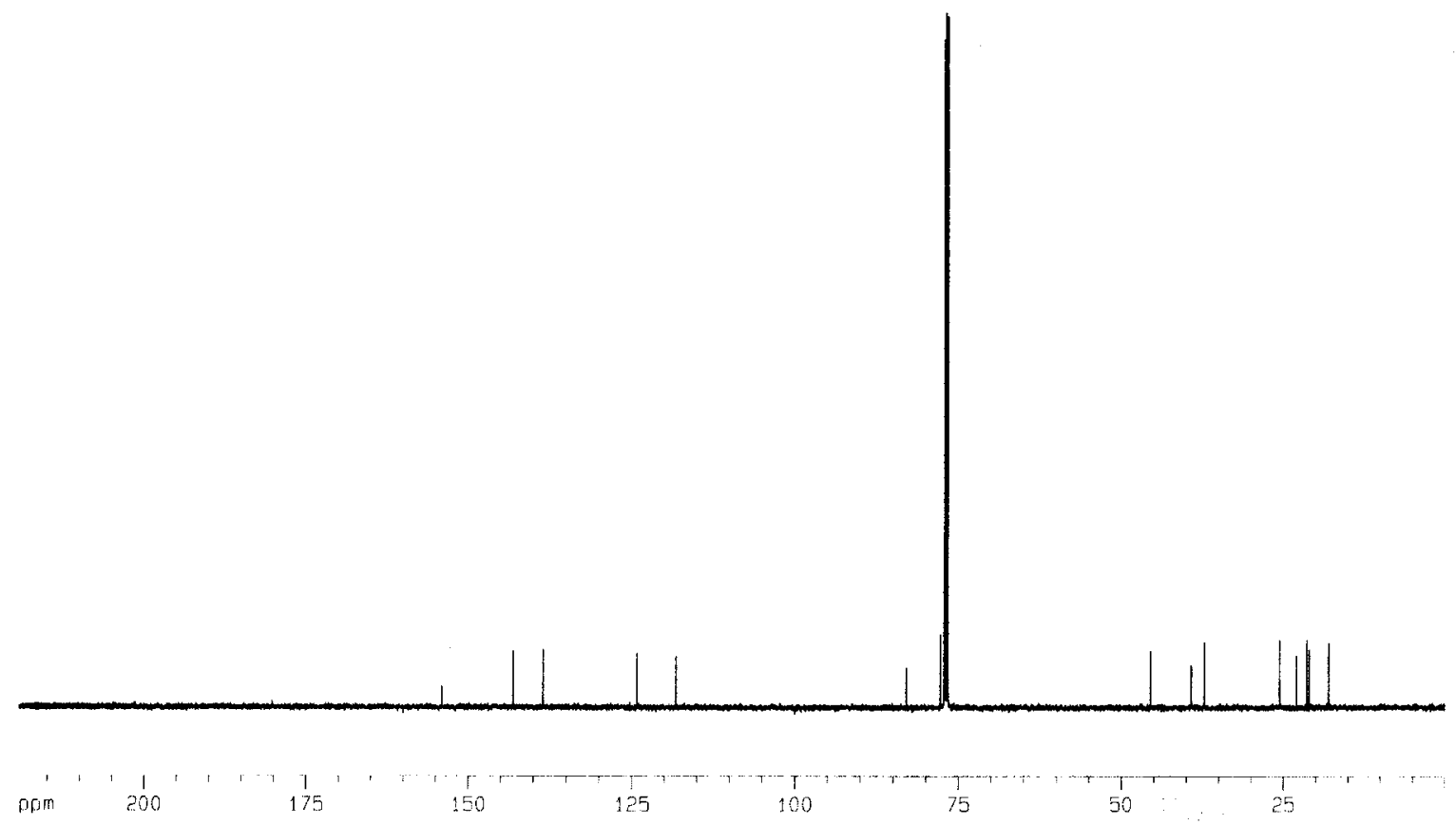


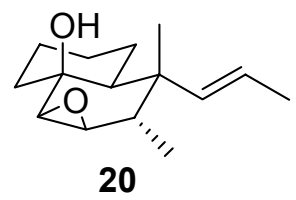

${ }^{1} \mathbf{H}$ NMR $\left(\mathrm{CDCl}_{3}, 500 \mathrm{MHz}\right)$

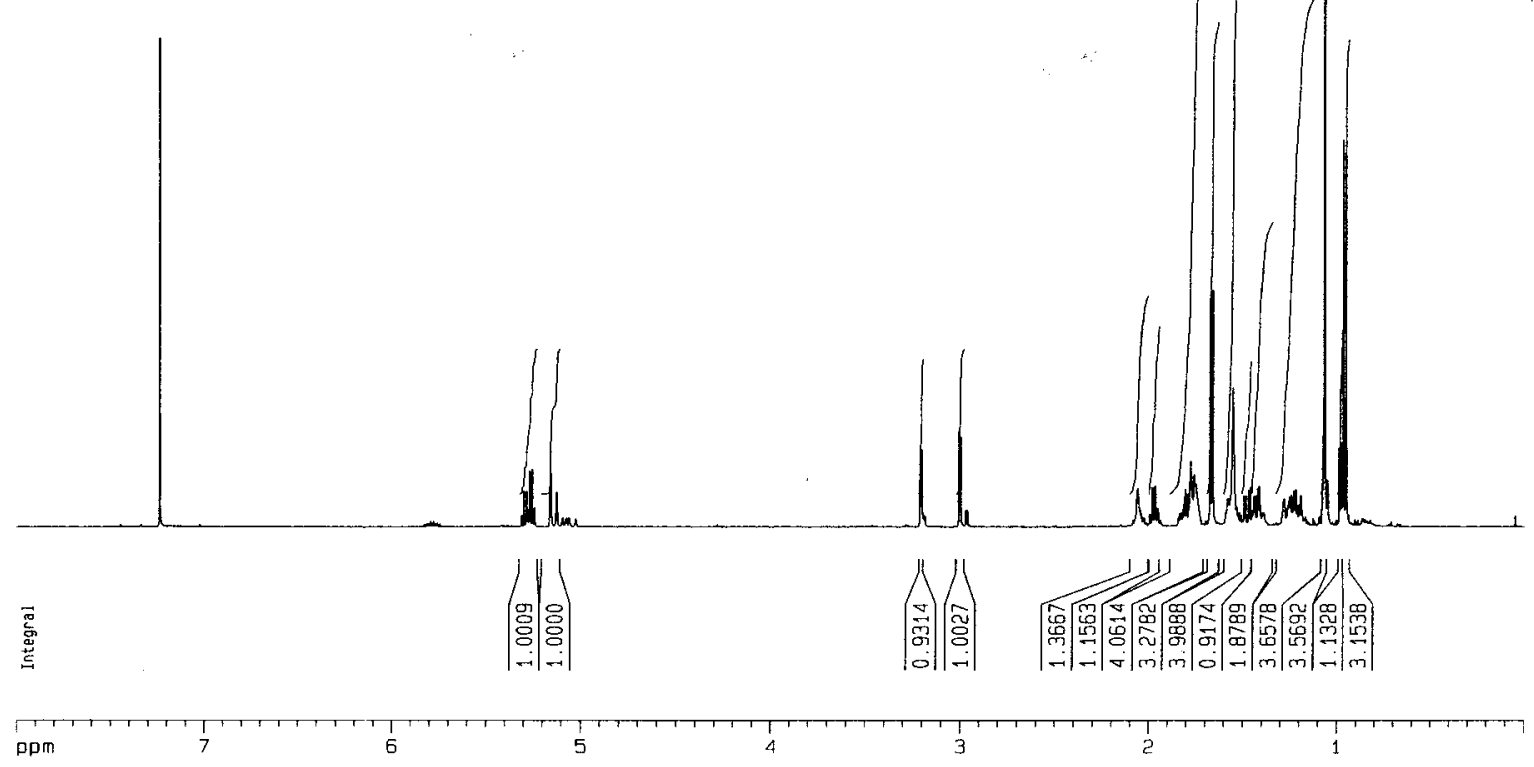

${ }^{13} \mathbf{C}$ NMR $\left(\mathrm{CDCl}_{3}, 125 \mathrm{MHz}\right)$

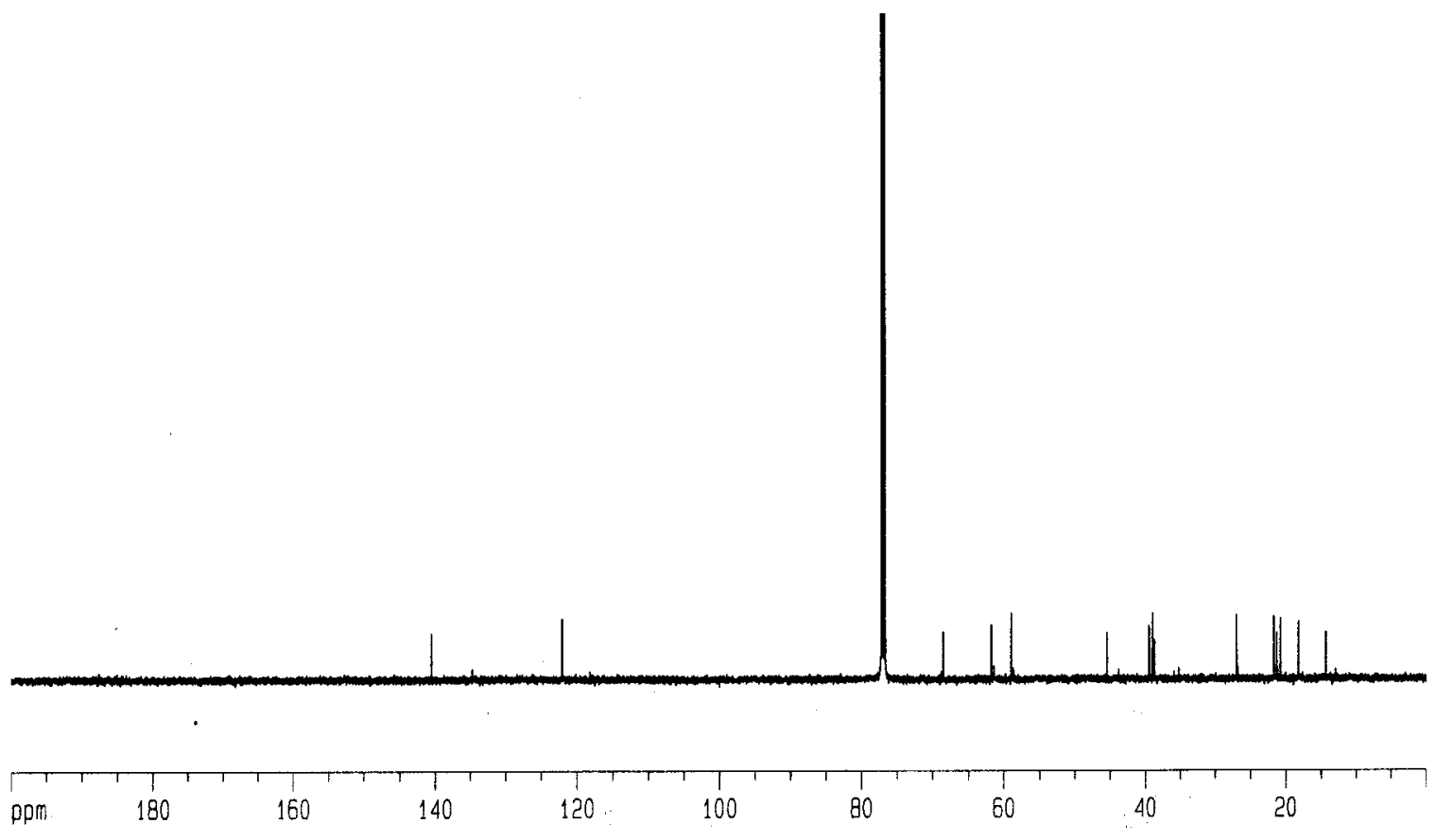

S38 


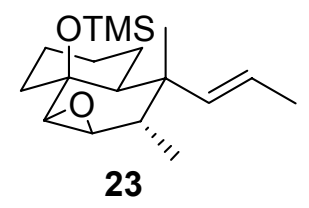

${ }^{1} \mathbf{H} \mathbf{N M R}\left(\mathrm{CDCl}_{3}, 500 \mathrm{MHz}\right)$

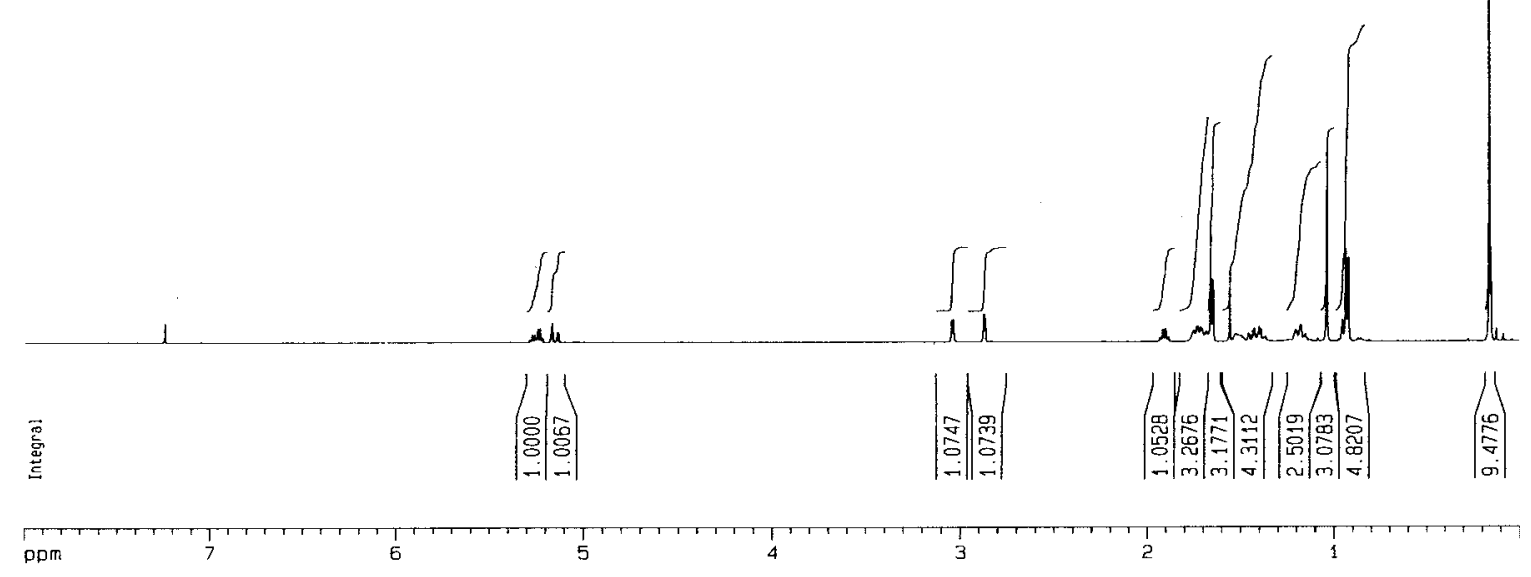

${ }^{13} \mathbf{C}$ NMR $\left(\mathrm{CDCl}_{3}, 125 \mathrm{MHz}\right)$

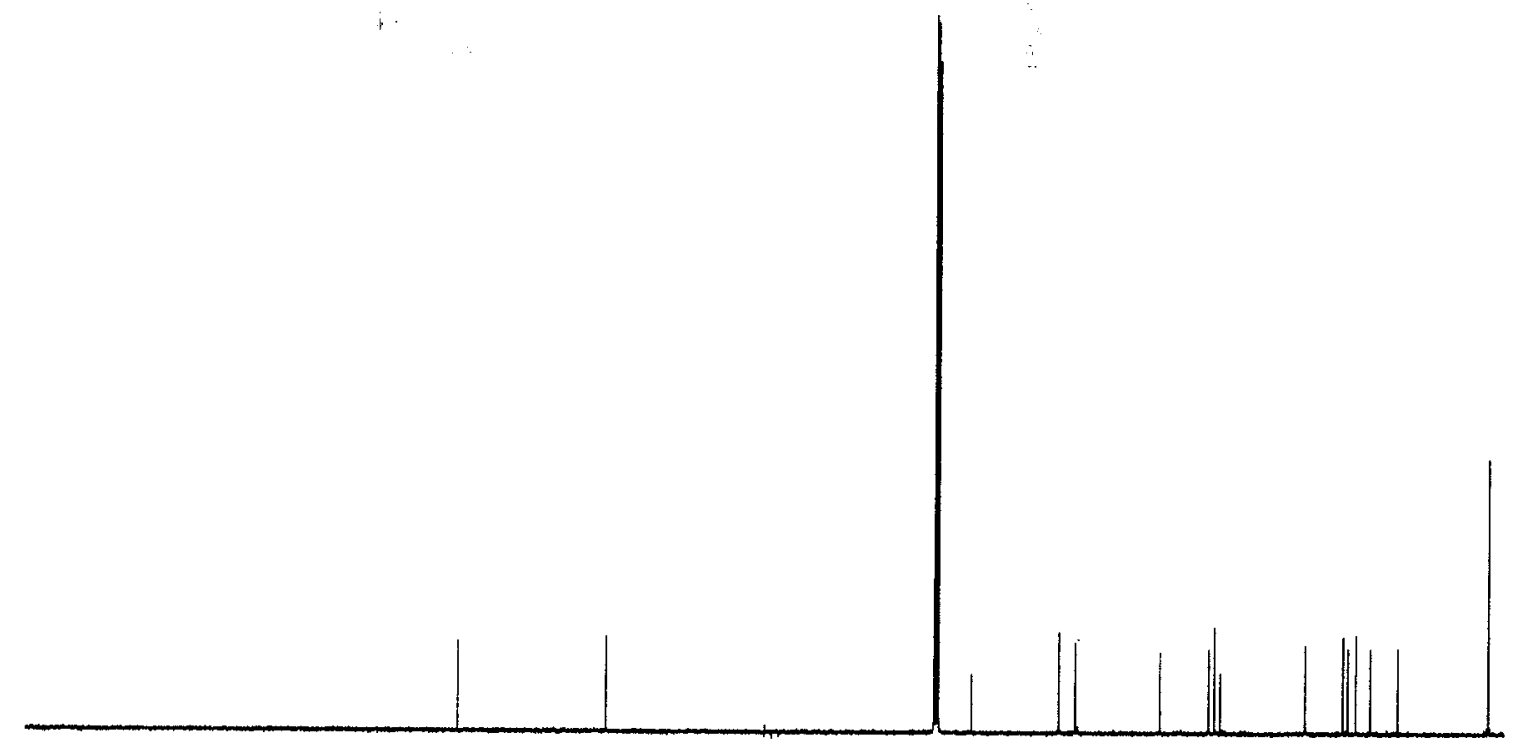

ppm

160
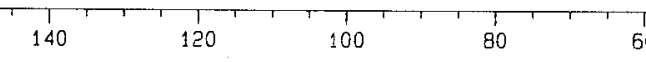

60 


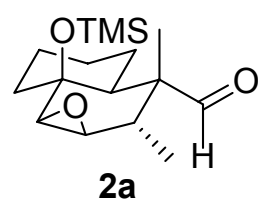

${ }^{1} \mathbf{H} \mathbf{N M R}\left(\mathrm{CDCl}_{3}, 500 \mathrm{MHz}\right)$

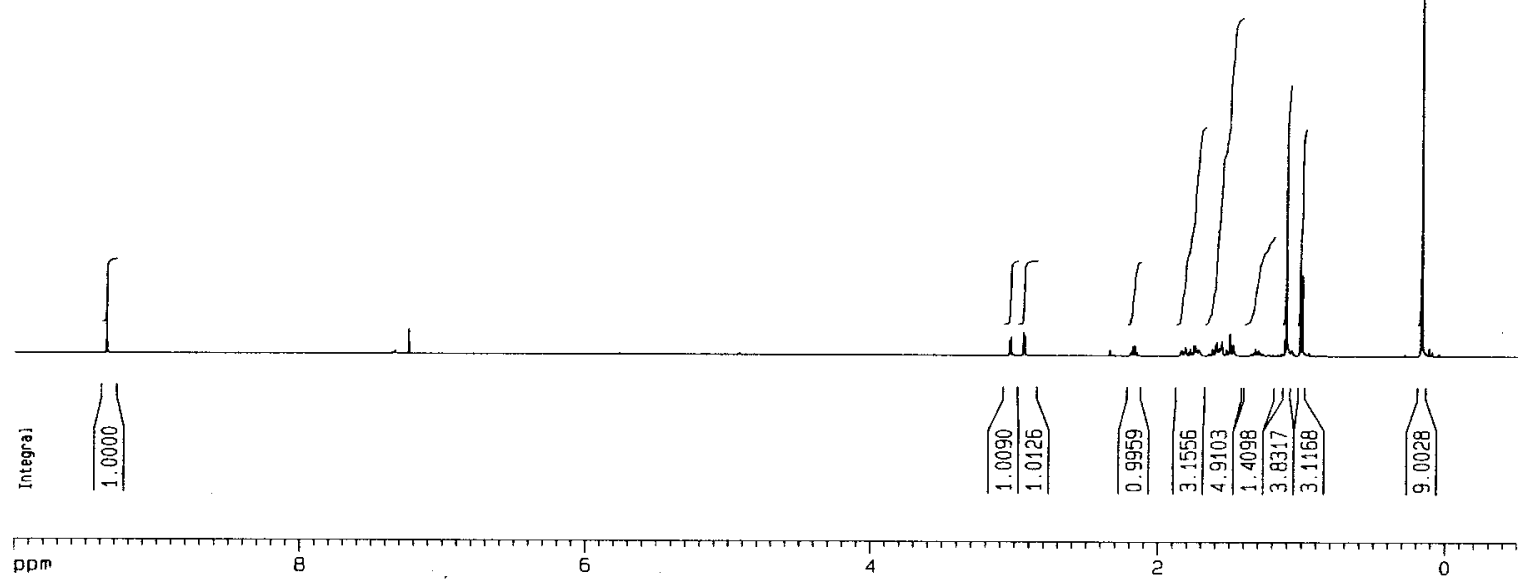

${ }^{13} \mathbf{C}$ NMR $\left(\mathrm{CDCl}_{3}, 125 \mathrm{MHz}\right)$

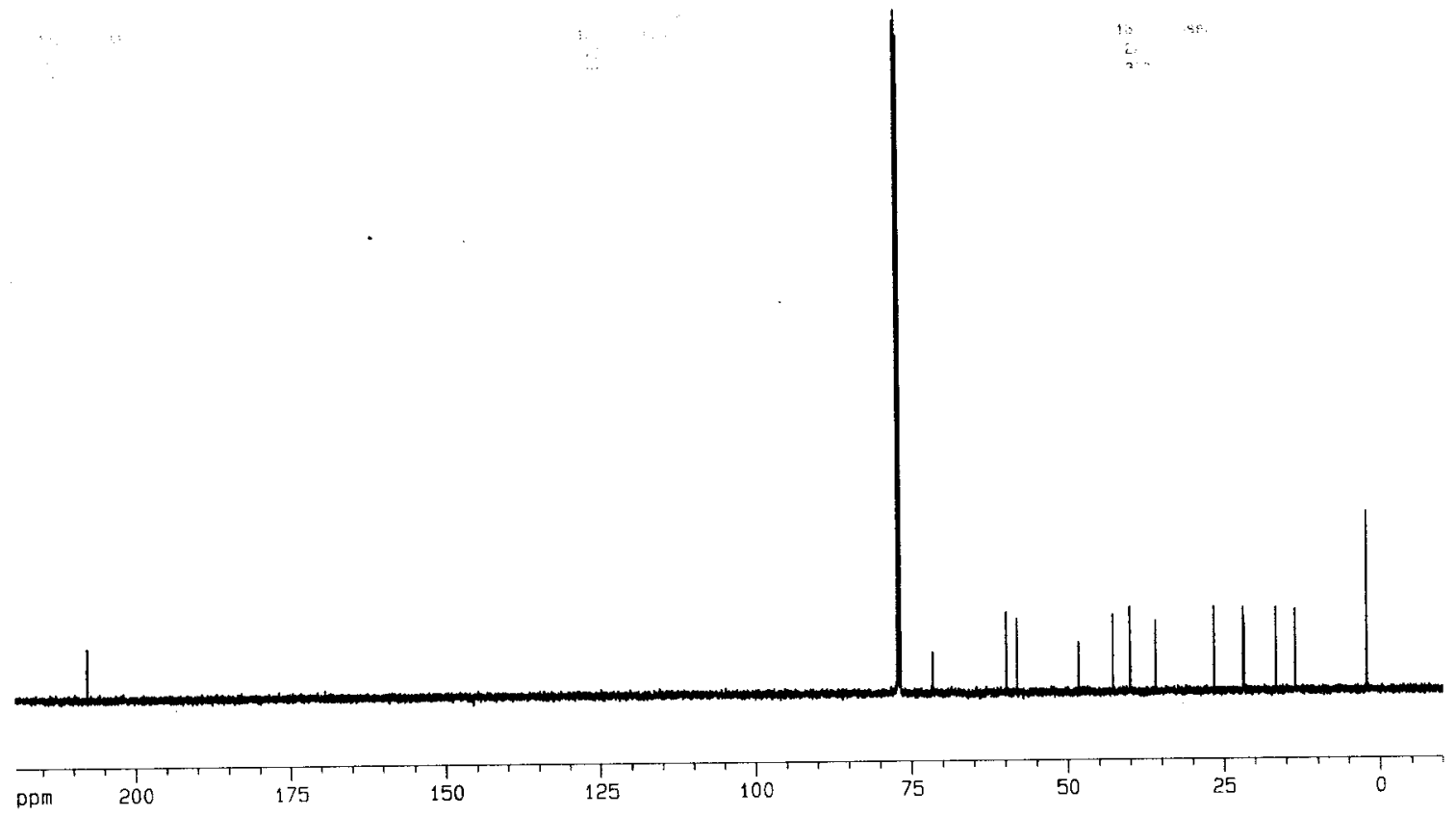




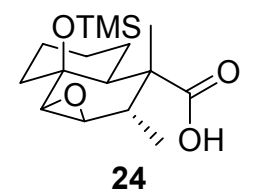

${ }^{1} \mathbf{H}$ NMR $\left(\mathrm{CDCl}_{3}, 500 \mathrm{MHz}\right)$

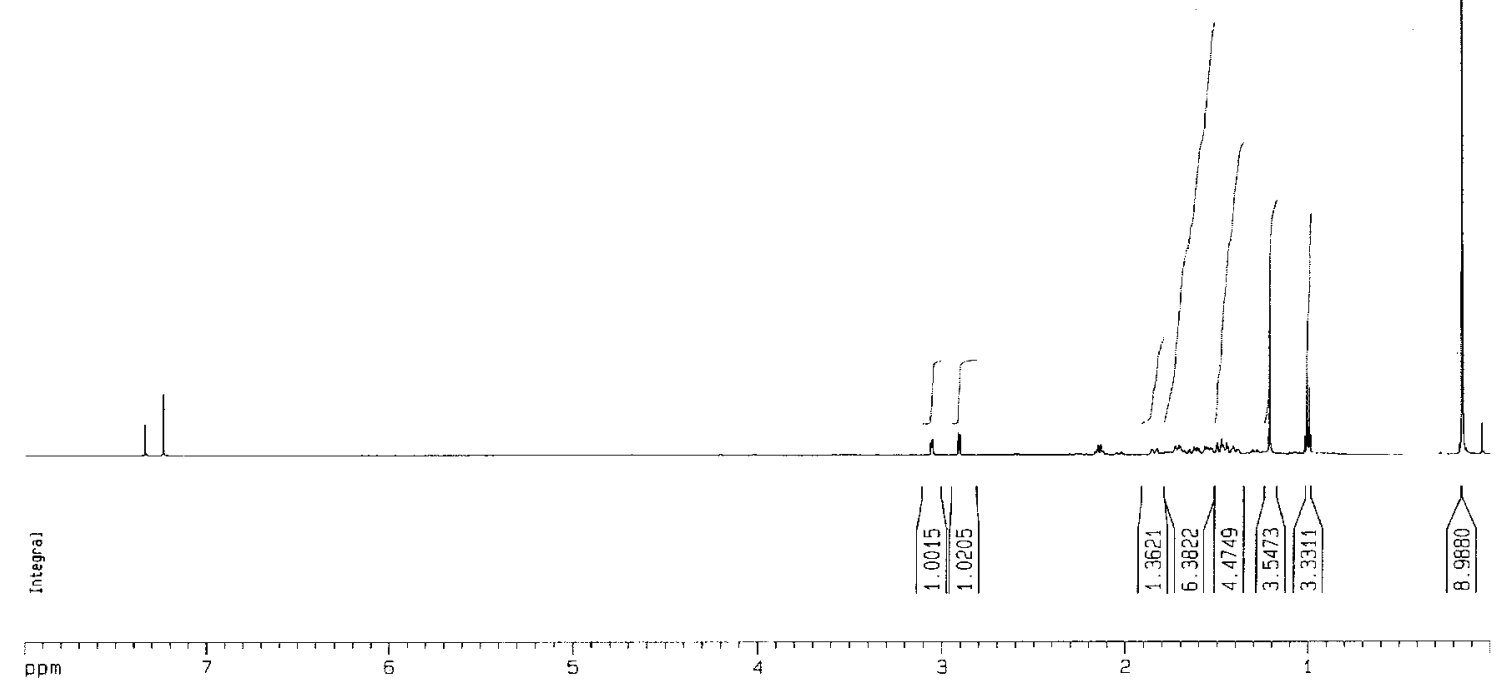

${ }^{13} \mathbf{C}$ NMR $\left(\mathrm{CDCl}_{3}, 125 \mathrm{MHz}\right)$

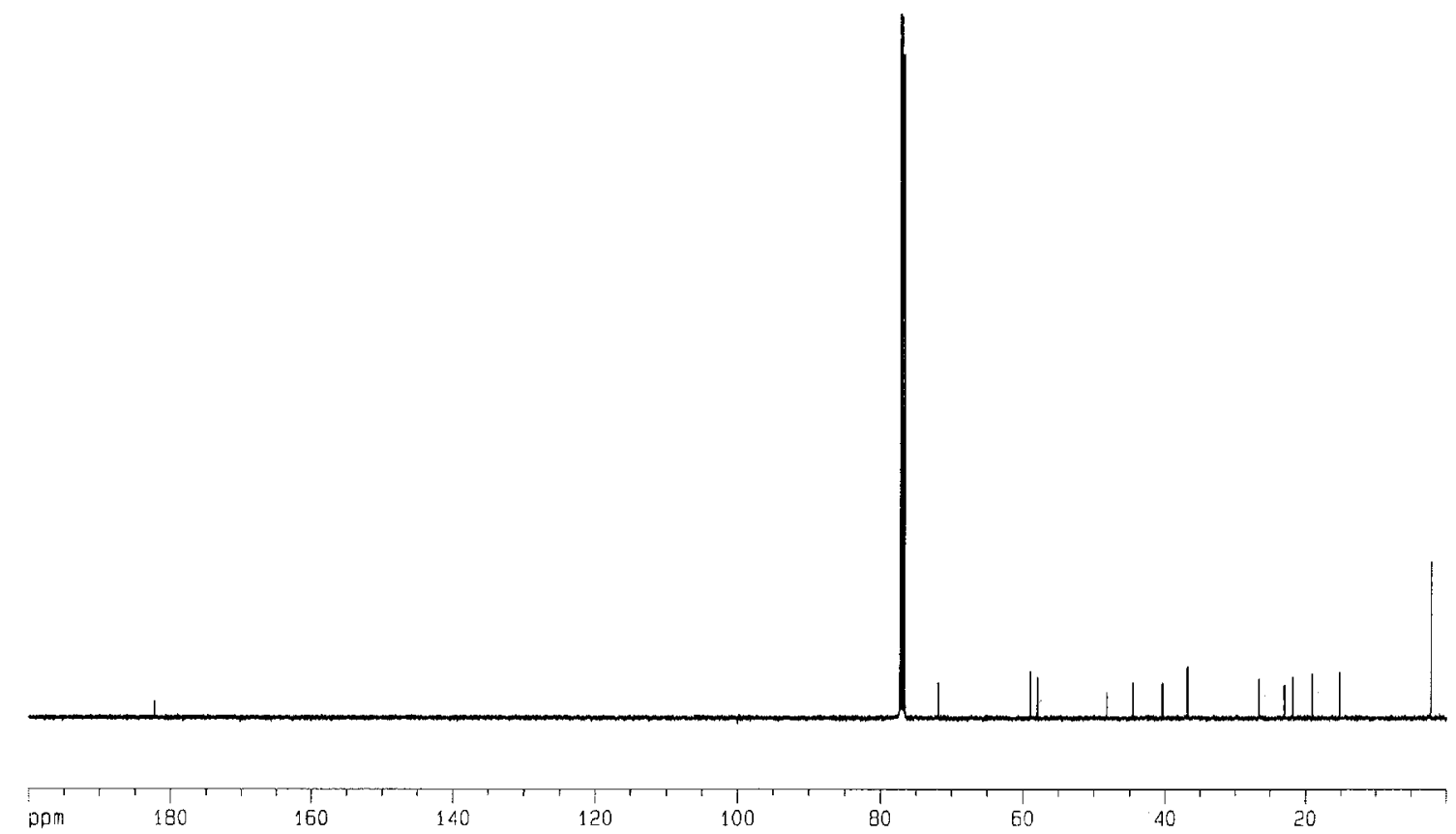




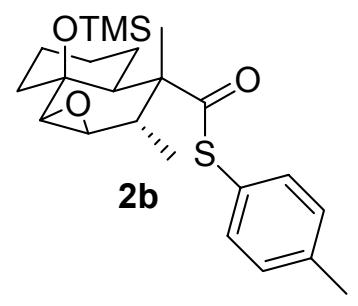

${ }^{\mathbf{1}} \mathbf{H} \mathbf{N M R}\left(\mathrm{CDCl}_{3}, 500 \mathrm{MHz}\right)$

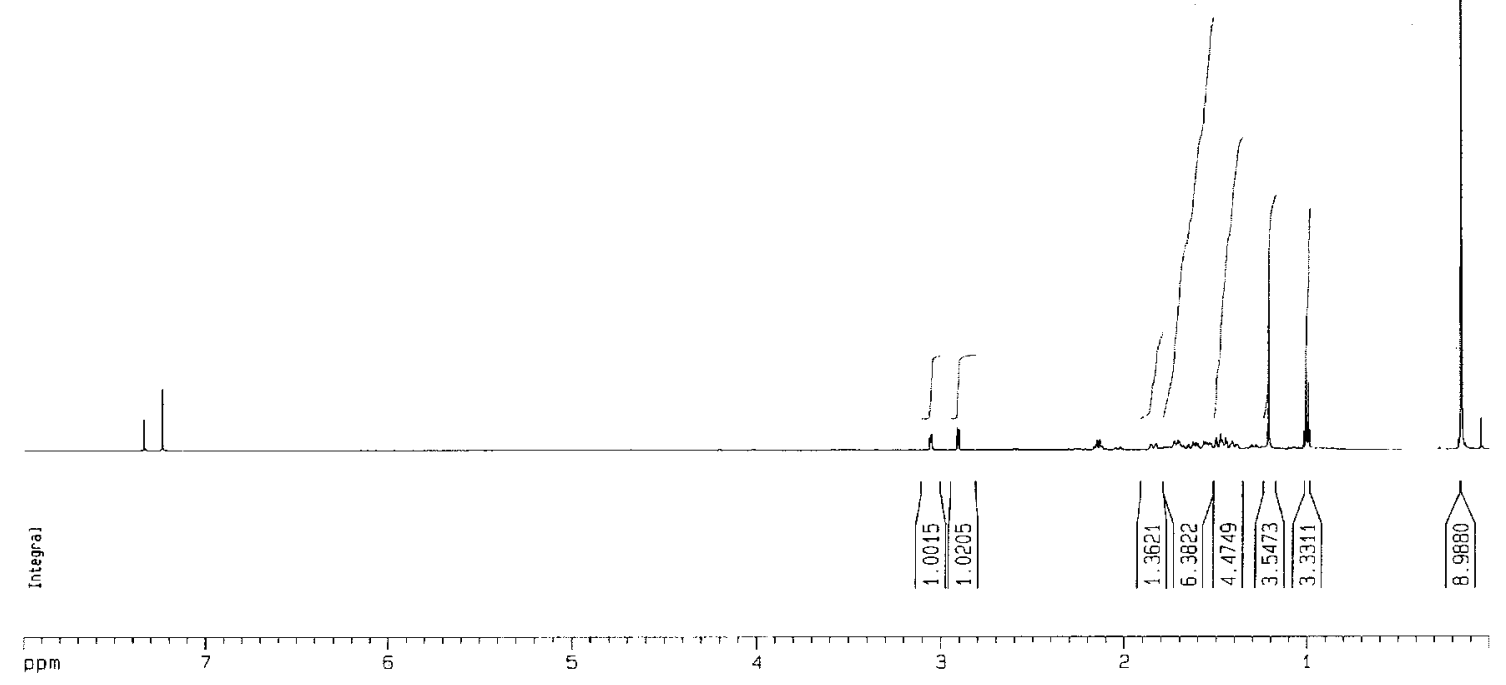

${ }^{13} \mathbf{C}$ NMR $\left(\mathrm{CDCl}_{3}, 125 \mathrm{MHz}\right)$

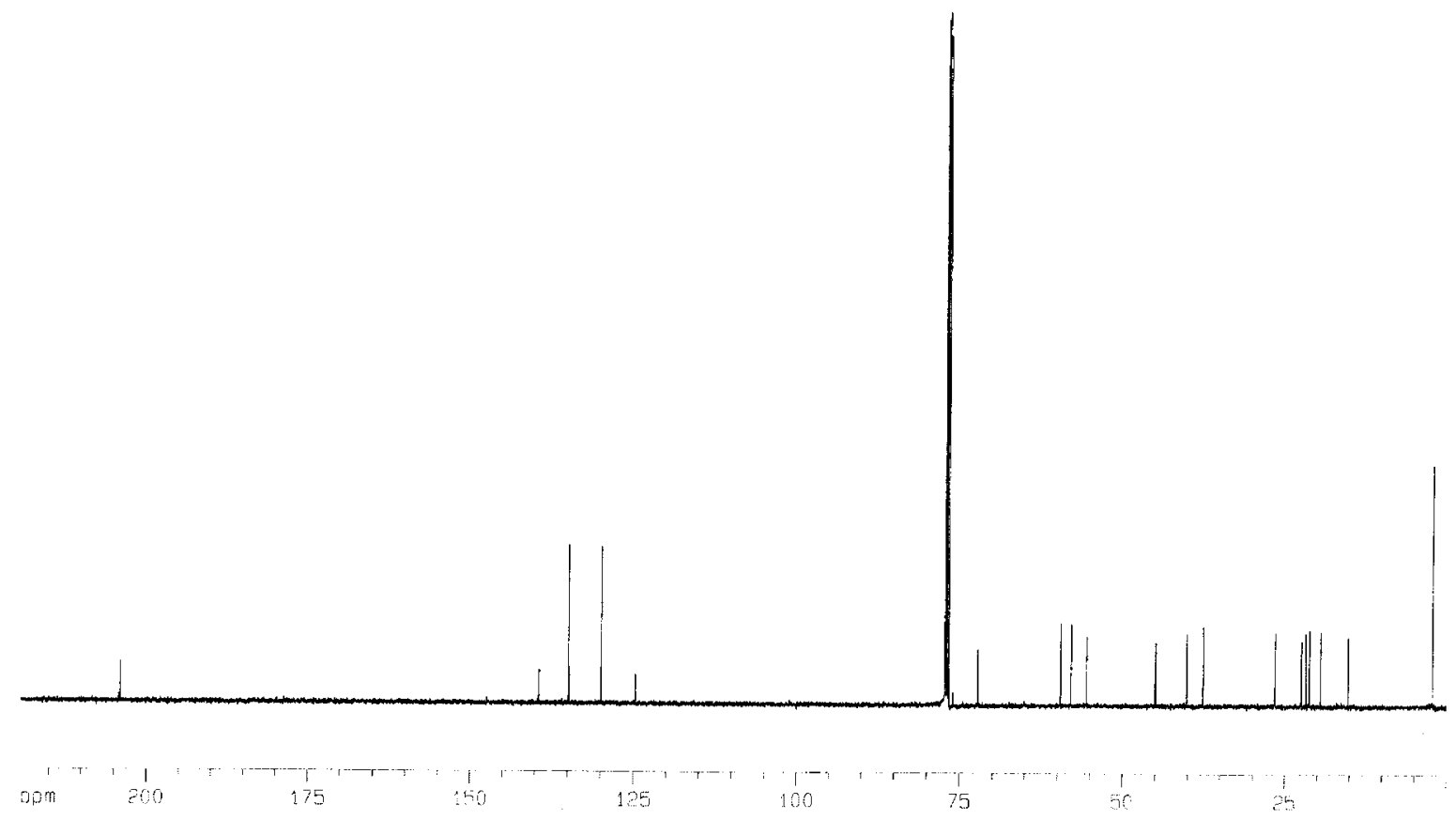




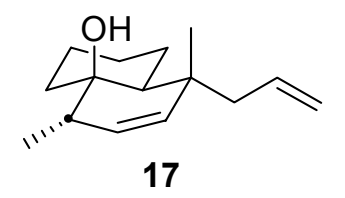

HMBC $\left(\mathrm{CDCl}_{3}\right)$

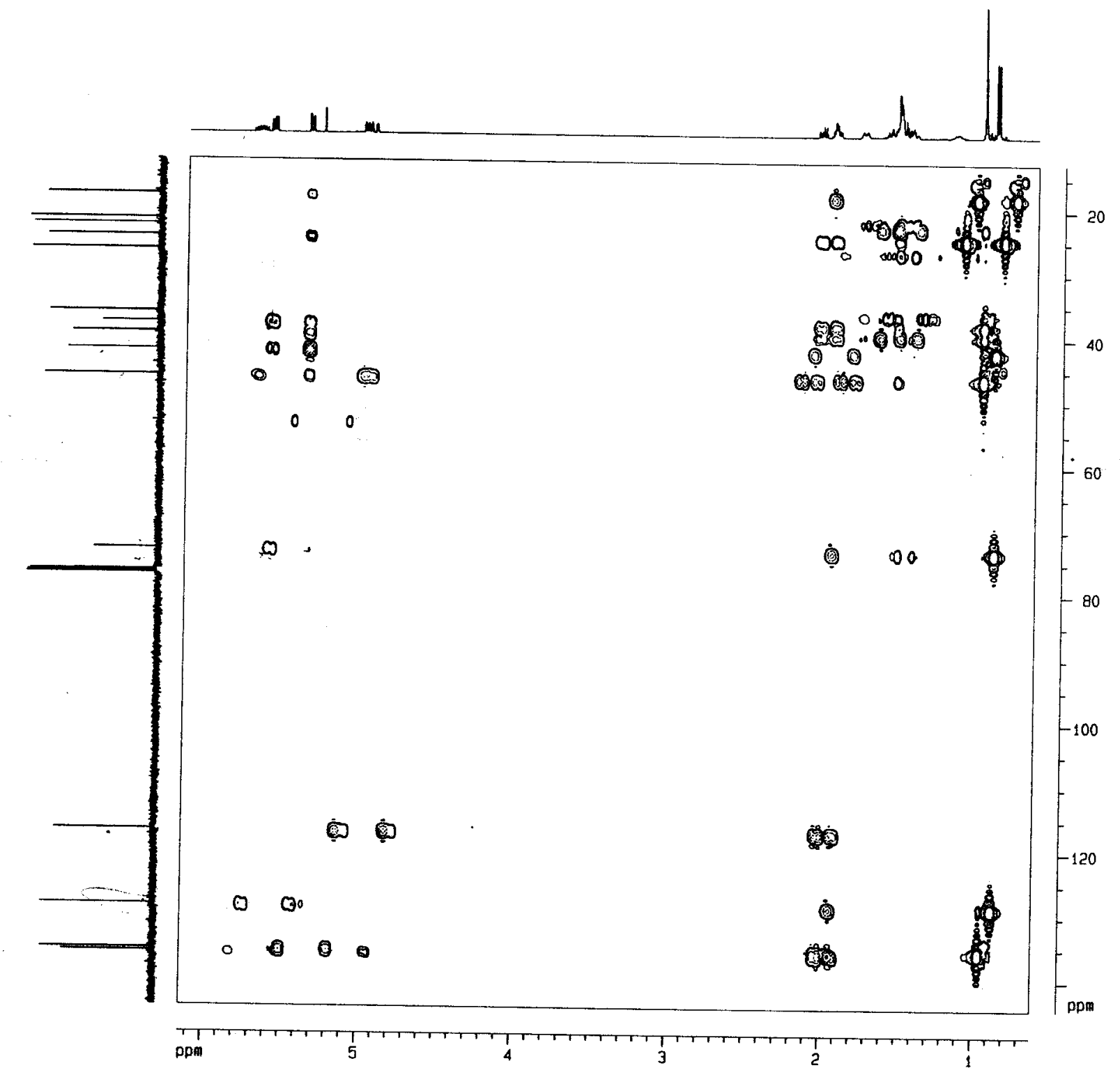




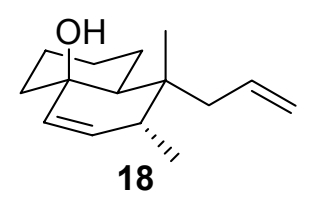

HMBC $\left(\mathrm{CDCl}_{3}\right)$

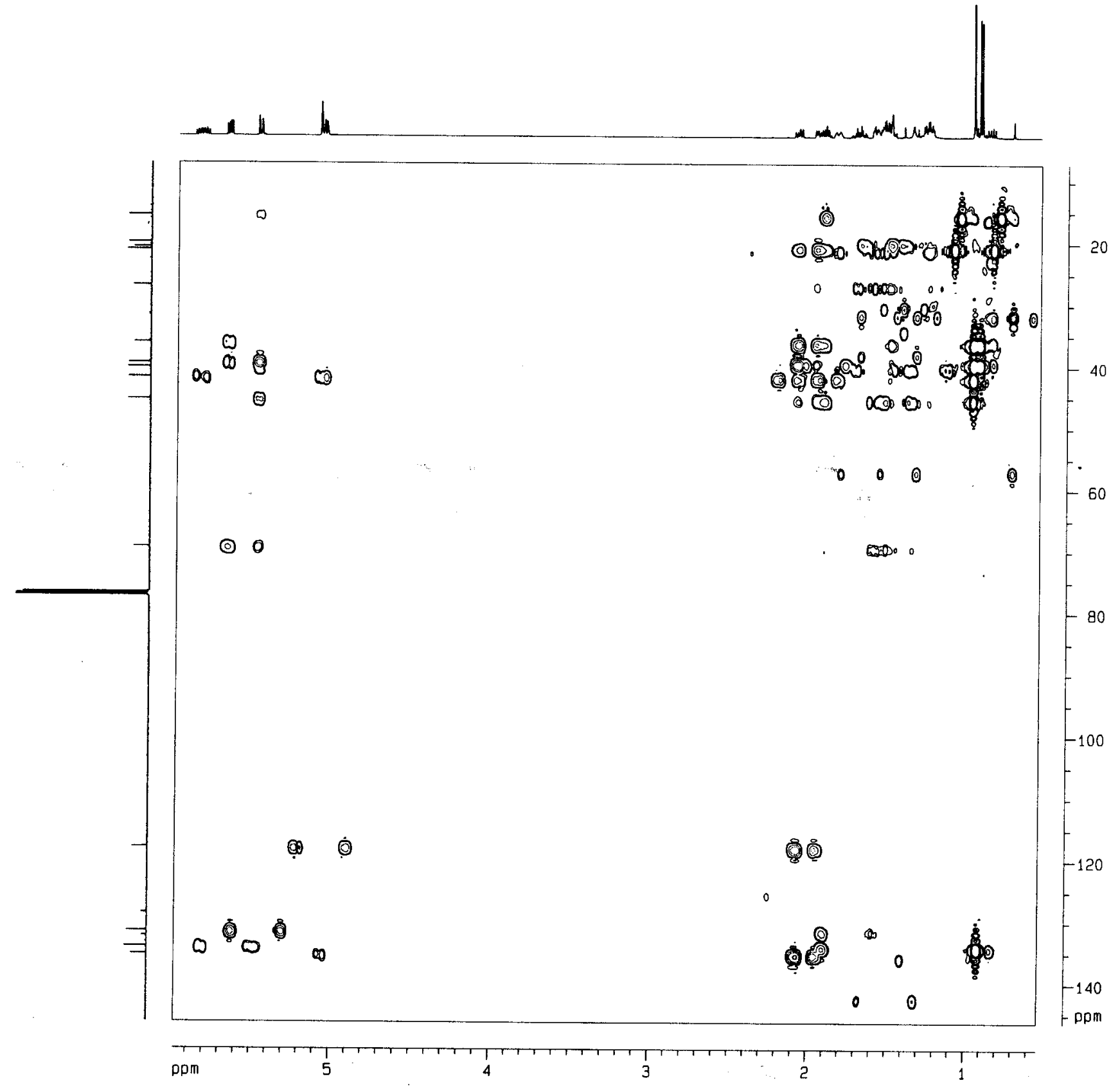




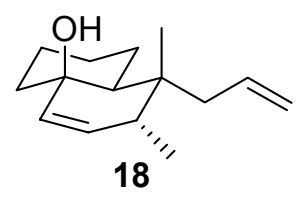

${ }^{1} \mathbf{H}$ NOESY $\left(\mathrm{CDCl}_{3}\right)$

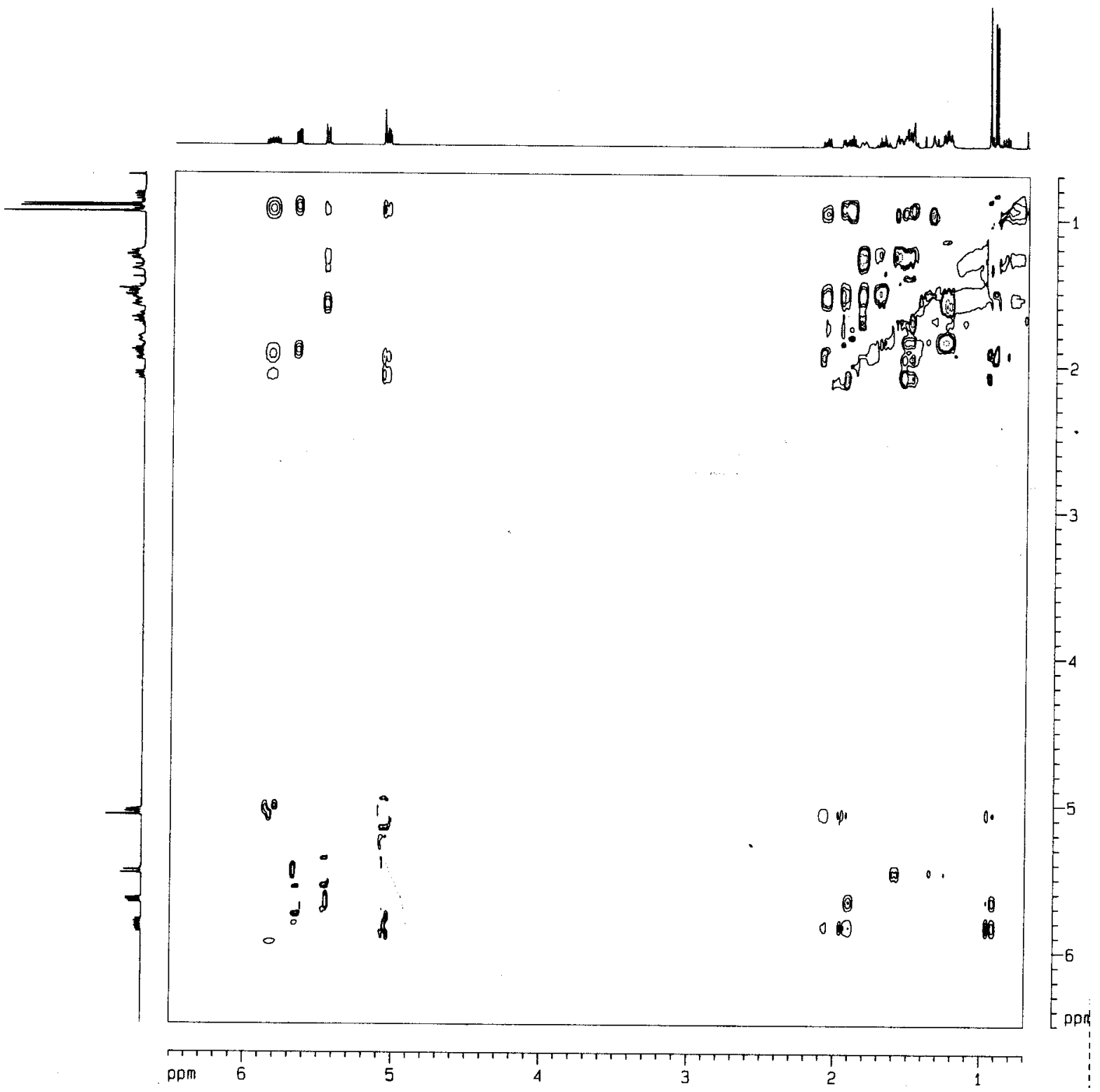



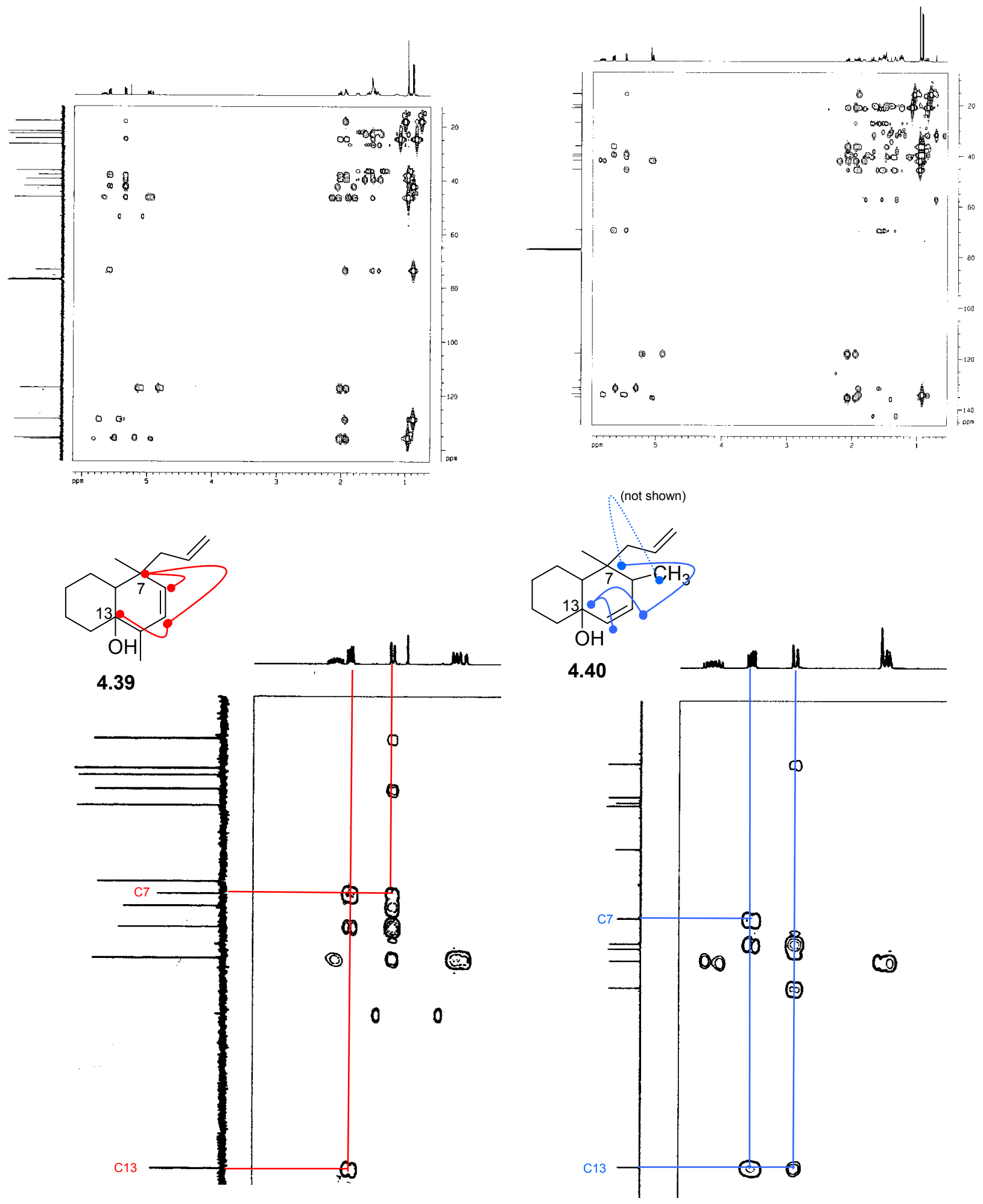

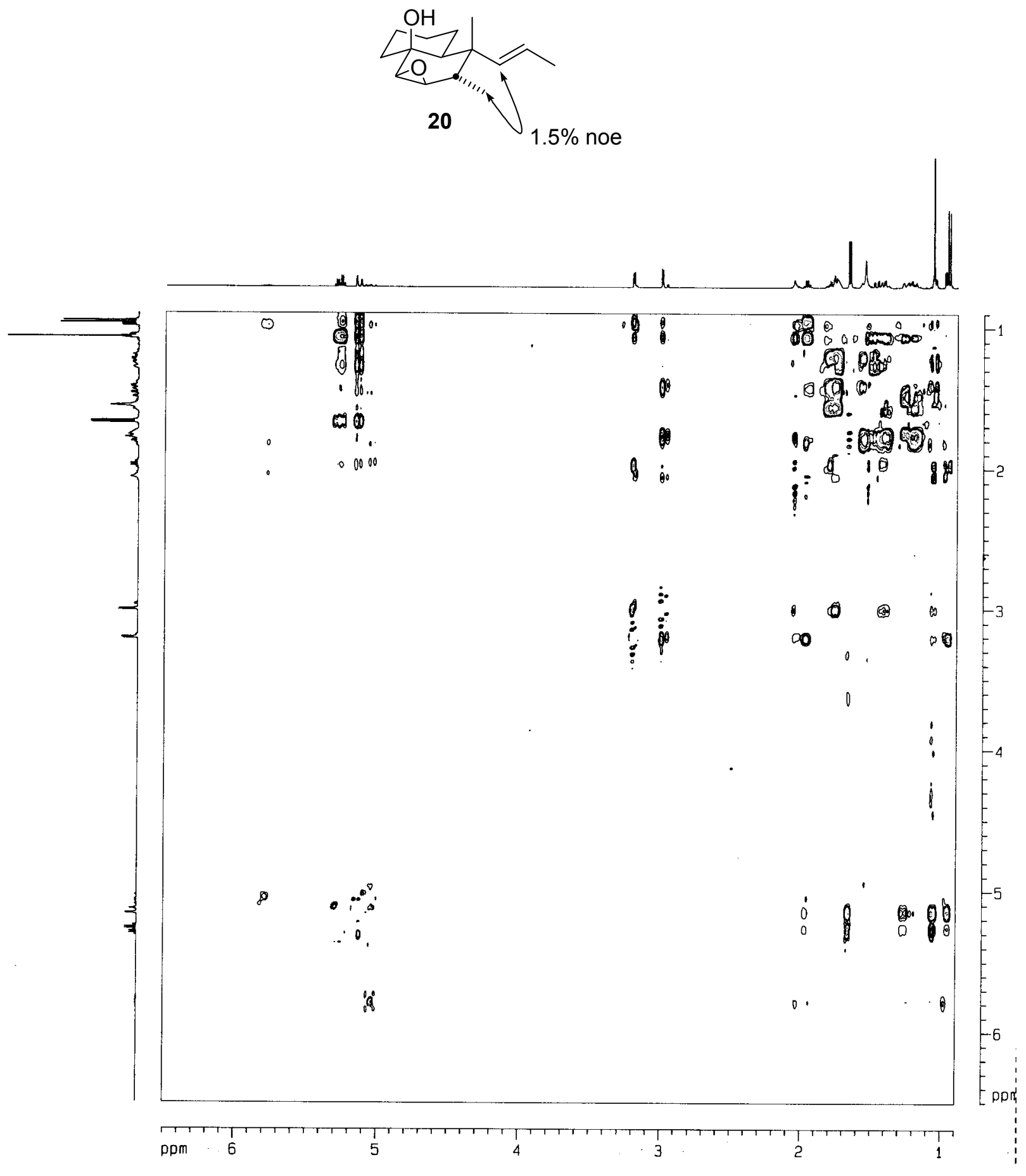